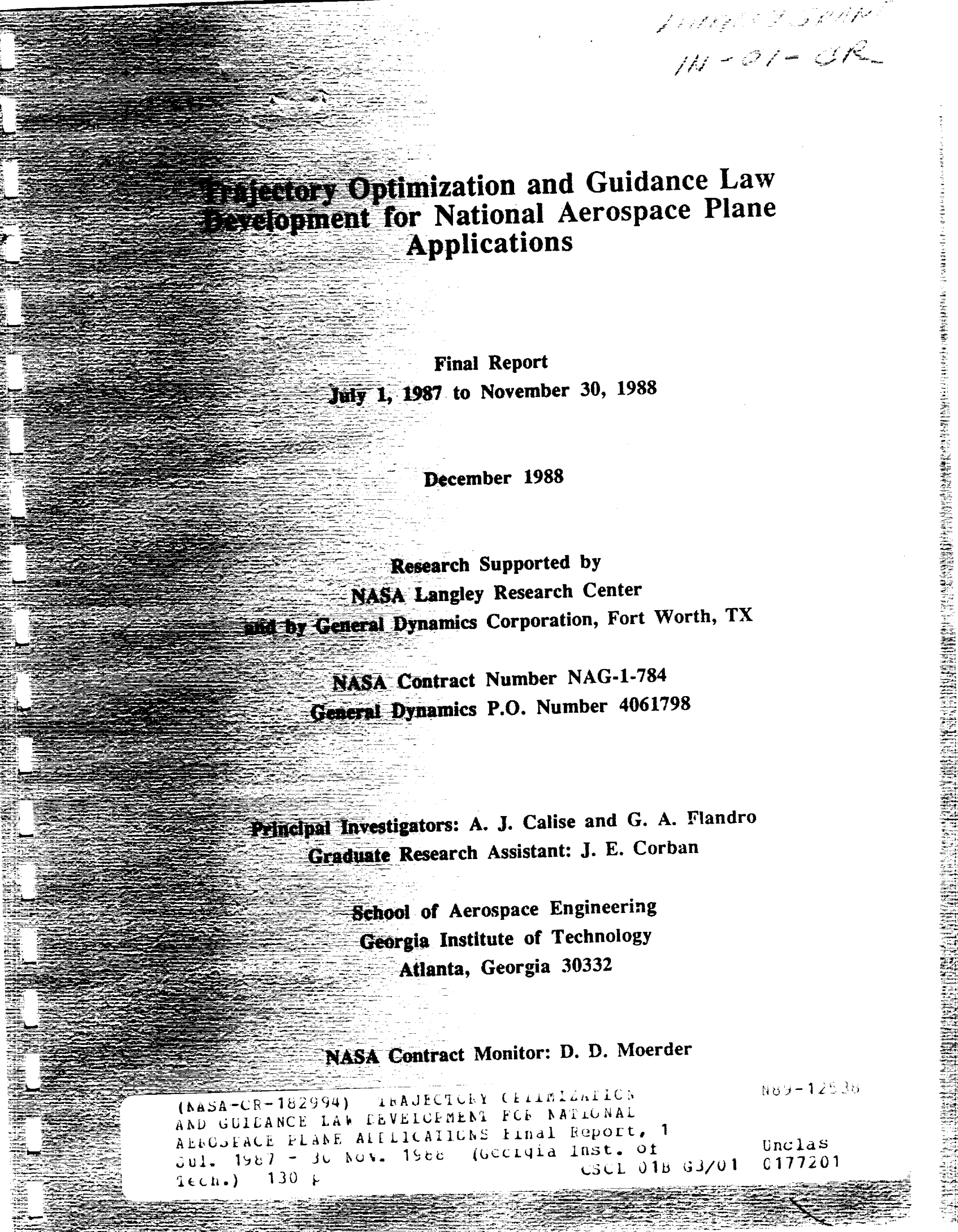




\title{
Trajectory Optimization and Guidance Law Development for National Aerospace Plane Applications
}

\author{
Final Report \\ July 1,1987 to November 30,1988
}

December 1988

\author{
Research Supported by \\ NASA Langley Research Center \\ and by General Dynamics Corporation, Fort Worth, TX \\ NASA Contract Number NAG-1-784 \\ General Dynamics P.O. Number 4061798
}

Principal Investigators: A. J. Calise and G. A. Flandro Graduate Research Assistant: J. E. Corban

School of Aerospace Engineering Georgia Institute of Technology Atlanta, Georgia 30332

NASA Contract Monitor: D. D. Moerder 


\section{Table of Contents}

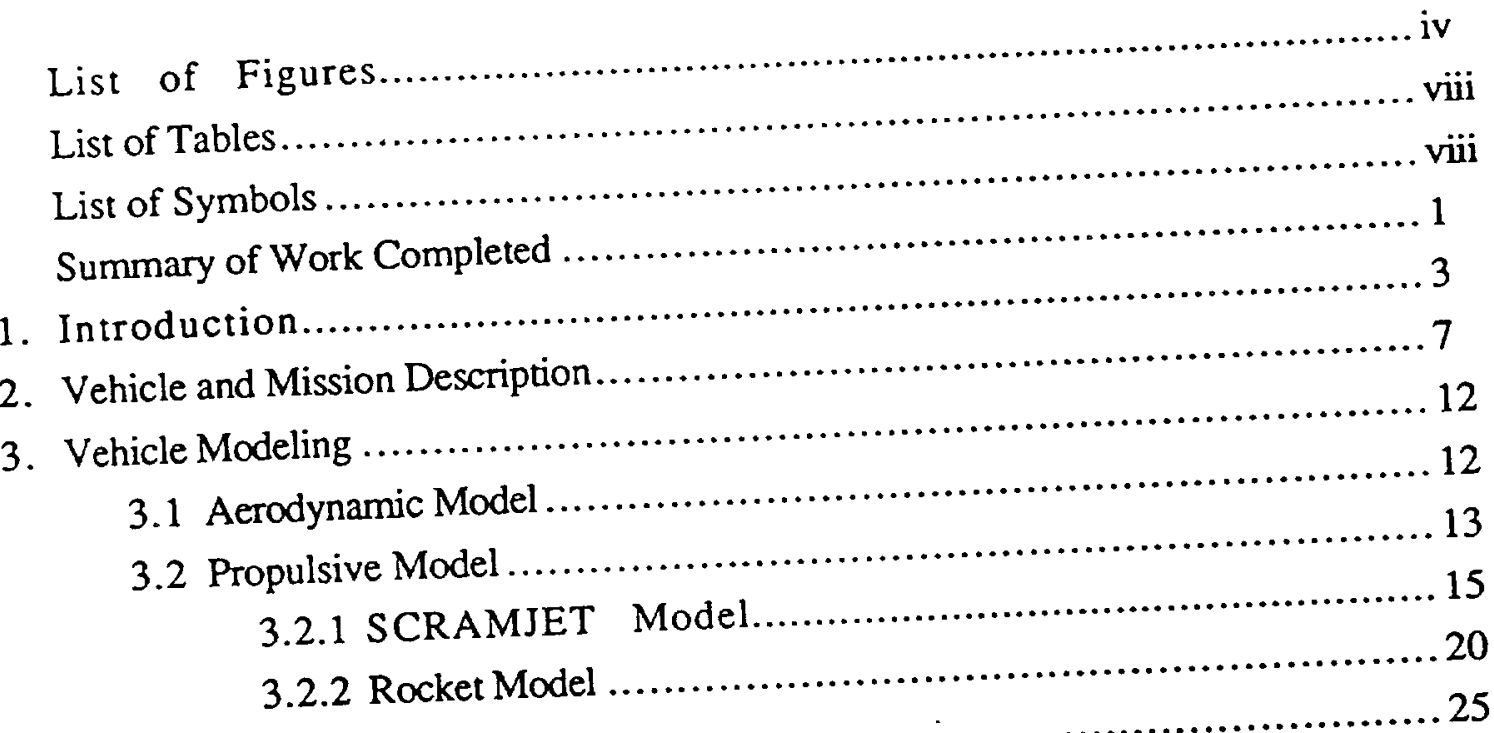

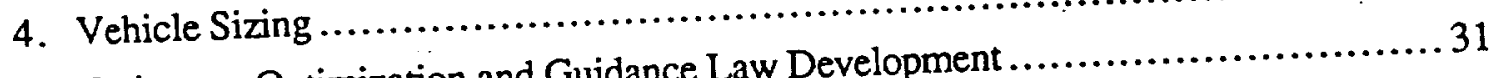

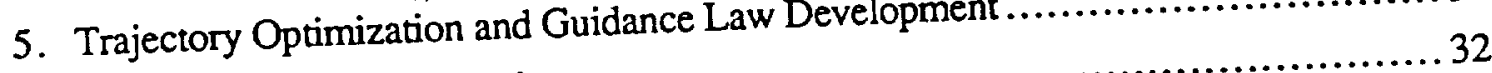

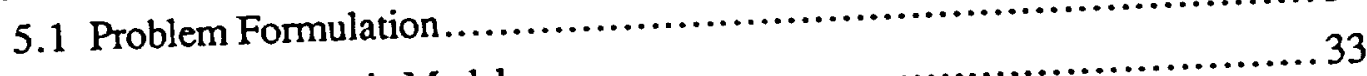

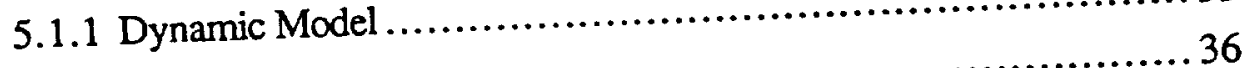

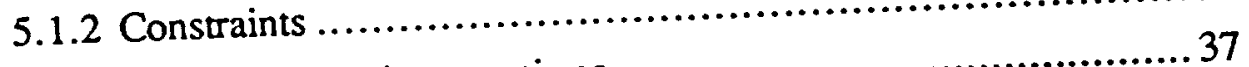

5.1.3 Simplifying Assumptions........................................4 42

5.2 Application of Singular Perturbation Theory ............................... 44

5.2.1 Reduced Solution...........................44

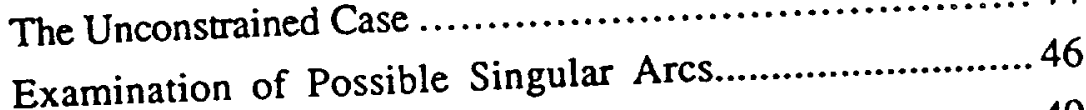

Analytic Form for Throttle Switching Condition...............49

Estimation of States and Costates in the Reduced Solution ..... 50

Inclusion of a Maximum Dynamic Pressure Constraint ........ 52

Reduced Solution: Numerical Results ...................... 53

5.2 .2 Boundary Layer Analysis.....................................6 60

The Unconstrained Case ...................................6 60

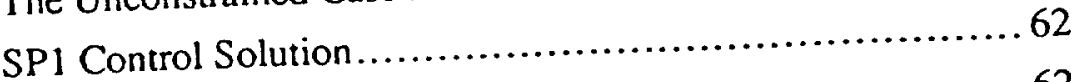

SP2 Control Solution...................................62 
NLT Control Solution ................................ 71

Inclusion of a Maximum Dynamic Pressure Constraint ........ 79

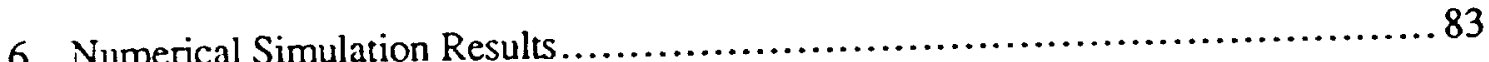

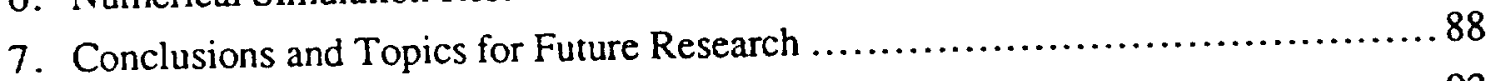

References ............................................................. 93

Appendix A: Prediction of Lift and Drag in Hypersonic Flow ....................... 1 Appendix B: Minimization of a Hamiltonian Function with One Unknown Costate and One Costate of Known Sign .................................... 1 


\section{List of Figures}

Page

Figure

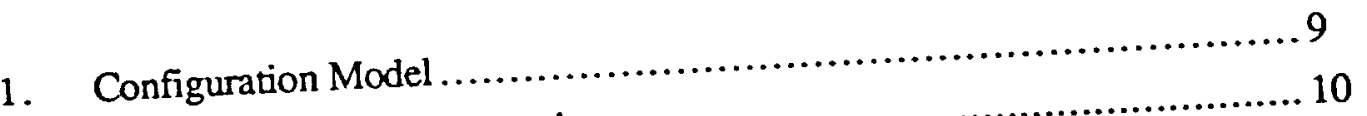

2. Model General Dimensions......................................... 14

3. Drag Polar, experimental results for trimmed vehicle at Mith angle of attack for trimmed

4. Measured variation of lift coefficient with angle of attack for trimmed

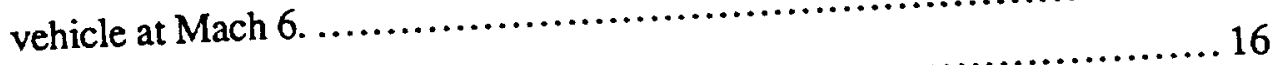

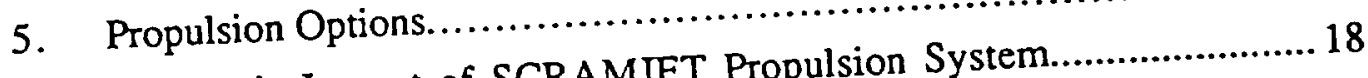

6. Schematic Layout of SCRAMJET Propulsion

7. Predicted SCRAMJET Fuel Specific Impul Consumption......................21

8. Predicted SCRAMJET Thrust Specific Fuel Consumpton.....22

9. Predicted SCRAMJET Thrust.................................22

10. Predicted SCRAMJET Fuel-to-Air Ratio .......................... 23

11. Predicted rocket performance, fuel specific implsevers

12. Predicted rocket performance, thrust versus altitude, .............24

13. Predicted rocket performance, thrust specific ........................24

14. Predicted rocket performance, mass flow rate ..........

15. Level Flight Envelope (SCRAMJET only) as a function of Engine $\ldots .27$ Inlet Area, $\mathrm{L}=\mathrm{W}$.

16. Level Flight Envelope (SCRAMJET only) as a function of Engine Inlet Area, $\mathrm{L}=\mathrm{W}-\mathrm{C} . \mathrm{F}$

17. Level Flight Envelope (SCRAMJET only) as a function of vehicle weight.........................................

18. Level Flight Envelope for a combination of SCRAMJET and rocket

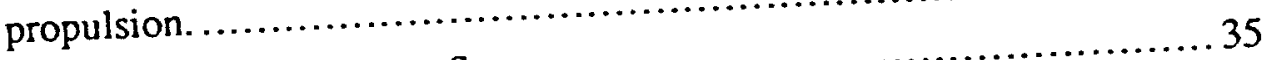

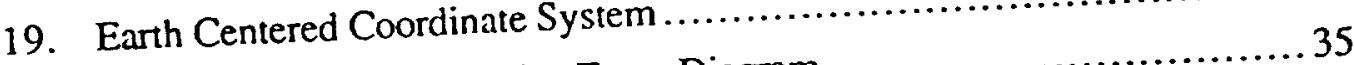

21. Reduced Solution, Innconstrained Fuel-Optimal Ascen 35 
22. Reduced Solution, q Constrained Fuel-Optimal Ascent Trajectories....... 57

23. Reduced Solution, Mechanism by which altitude discontinuity occurs for q constrained case, SCRAMJET only. ............................ 57

24. Reduced Solution, Required Lift Coefficient computed along the $q$ constrained path.................................................. 58

25. Reduced Solution, Required Angle of Attack computed along the $q$ constrained path........................................................ 58

26. Reduced Solution, Thrust to Drag ratio computed along the unconstrained path.......................................................55

27. Reduced Solution, Thrust to Drag ratio computed along the $\mathrm{q}$ constrained path.........................................................5 59

28. Reduced Solution, History of Flight Path Angle estimated along $q$ constrained path, SCRAMJET/rocket case.

29. SP1 Controller Tracking Constant Energy Reduced Solution, Altitude versus Time

30. SP1 Controller Tracking Constant Energy Reduced Solution, Flight Path Angle versus Time...................................................6 63

31. SP2 Controller Tracking Constant Energy Reduced Solution, Altitude versus Time

32. SP2 Controller Tracking Constant Energy Reduced Solution, Flight Path Angle versus Time. 68

33. SP2 Controller Tracking Constant Energy Reduced Solution, Altitude versus Time, $\mathrm{L} / \mathrm{W} \leq 2.0$

34. SP2 Controller Tracking Constant Energy Reduced Solution, Flight

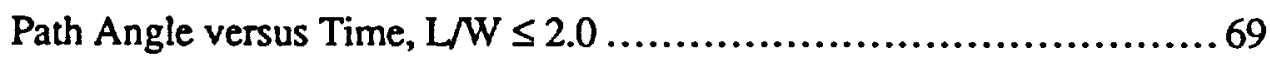

35. L/W for SP2 Controller Tracking Constant Energy Reduced Solution ..... 70

36. L/W for SP2 Controller Tracking Constant Energy Reduced Solution . . . . 70

37. Block Diagram Depicting Conceptual formulation of NLT Control Law ................................................................. 74

38. Block Diagram of Equivalant Linear System............................ 74

39. NLT Controller Tracking Constant Energy Reduced Solution, Altitude versus Time, Gains selected to match SP2 ............................ 75

40. NLT Controller Tracking Constant Energy Reduced Solution, Flight Path Angle versus Time, Gains selected to match SP2 
41. NLT Controller Tracking Constant Energy Reduced Solution, Altitude versus Time, $\mathrm{L} / \mathrm{W} \leq 2.0$, Gains selected to match $S P 2 \ldots \ldots \ldots \ldots \ldots \ldots \ldots 76$

42. NLT Controller Tracking Constant Energy Reduced Solution, Flight Path Angle versus Time, $\mathrm{L} / \mathrm{W} \leq 2.0$, Gains selected to match SP2 ........76

43. L/W for NLT Controller Tracking Constant Energy Reduced Solution, Gains selected to match SP2

44. L/W for NLT Controller Tracking Constant Energy Reduced Solution, $\mathrm{L} / \mathrm{W} \leq 2.0$, Gains selected to match SP2

45. NLT Controller Tracking Constant Energy Reduced Solution, Altitude versus Time, Gains picked to give a damping ratio of 0.7 and a $2 \%$ settling time of 120.0 seconds

46. NLT Controller Tracking Constant Energy Reduced Solution, Flight Path Angle versus Time, Gains picked to give a damping ratio of 0.7 and a $2 \%$ settling time of 120.0 seconds.

47. L/W for NLT Controller Tracking Constant Energy Reduced Solution, Gains picked to give a damping ratio of 0.7 and a $2 \%$ settling time of 120.0 seconds

48. NLT Controller Tracking Constant Energy q Constrained Reduced

Solution, Altitude versus Time, Gains picked to yield various damping ratios

49. NLT Controller Tracking q Constrained Constant Energy Reduced Solution, Flight Path Angle versus Time, Gains selected to yield various damping ratios

50. L/W for NLT Controller Tracking Constant Energy Reduced Solution, Gains selected to yield various damping ratios

51. NLT Guided Solution, Altitude and L/W Time Histories, with $\mathrm{dr}_{\mathrm{d}} / \mathrm{dt}=0$

52. NLT Guided Solution, Altitude and L/W Time Histories, with estimated $\mathrm{d} r_{\mathrm{o}} / \mathrm{dt}$

53. NLT Guided Solution, Flight Path Angle Time History, with $\mathrm{dr} d \mathrm{dt}=0$

54. NLT Guided Solution, Flight Path Angle Time History, with estimated $\mathrm{dr} / \mathrm{dt}$

A 1 Mach Number Independence 
A2 Comparison of pressure coefficients ............................. A5

A3 Drag Polar, predicted and measured values at $M=6.0$ (neutral elevon) ... A8

A4 Lift to Drag Ratio, predicted \& measured values at $M=6.0 \ldots \ldots \ldots \ldots \ldots$. A8

A5 Predicted Yaw Moment versus Sideslip with influence of Fin Cant Angle. A9

A6 Predicted effect of Elevon Deflection on Pitching Moment ..................A10

A7 Predicted and measured variation of Lift Coefficient with Angle of Attack at

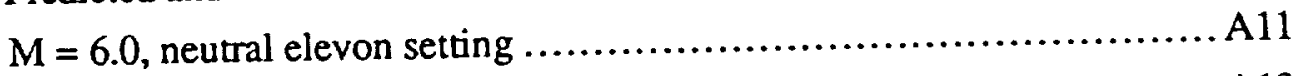

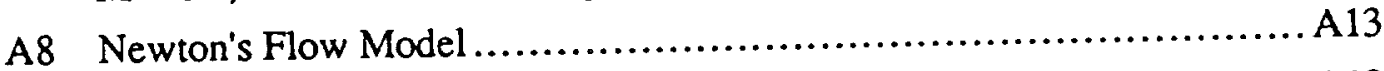

A9 Components of Lift and Drag for a Flat Plate ........................A13

A10 Severity of Bluntness.............................................. A 15

A11 2-D Representation of 3-D Flow Problem............................. A15 


\section{List of Tables}

Table

Page

1 Summary of Vehicle Configuration Features ............................. 8

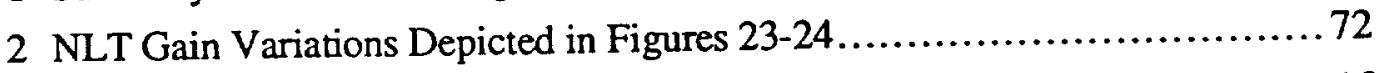

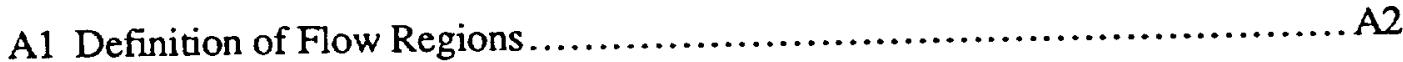

\section{List of Symbols}

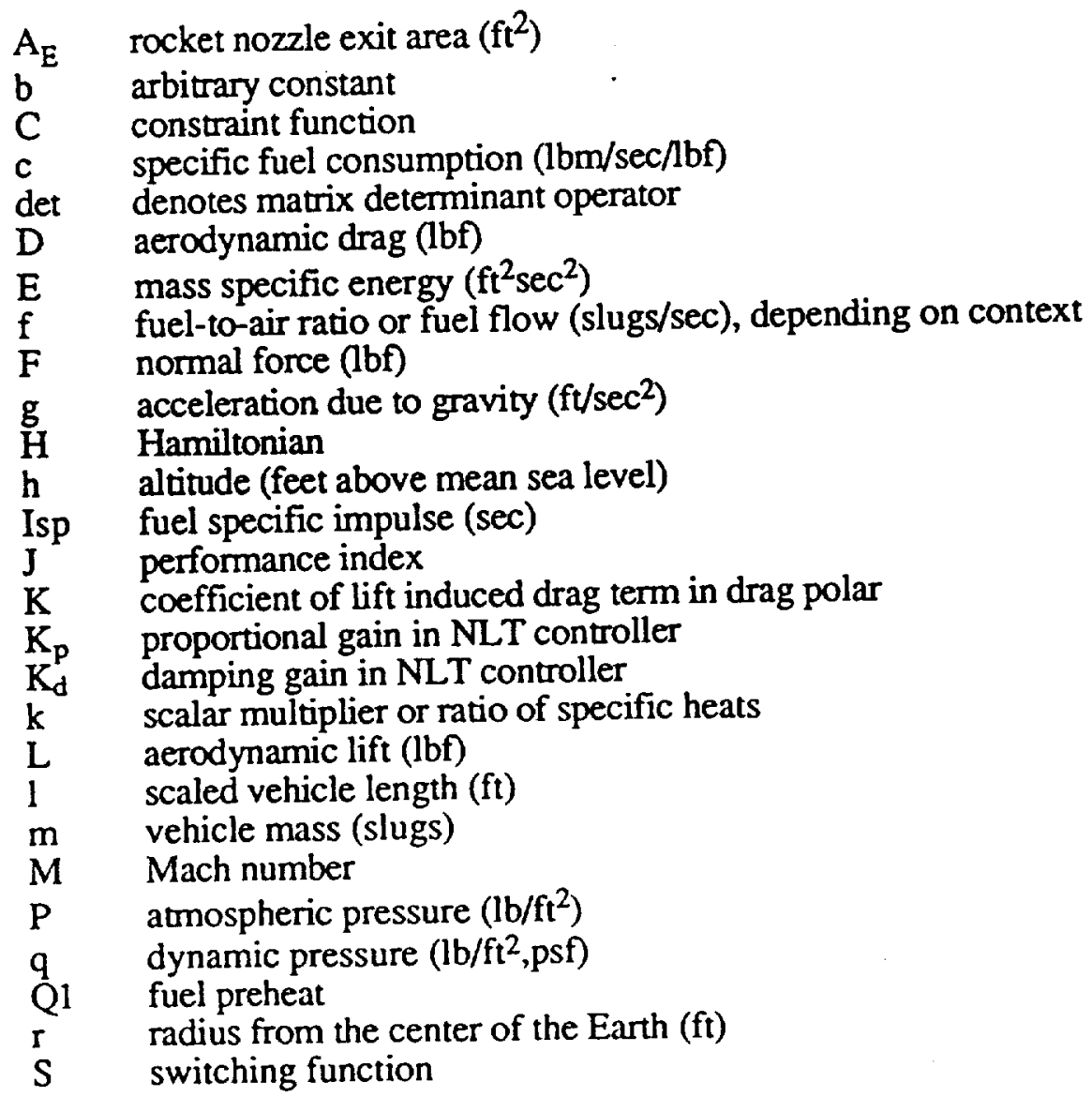




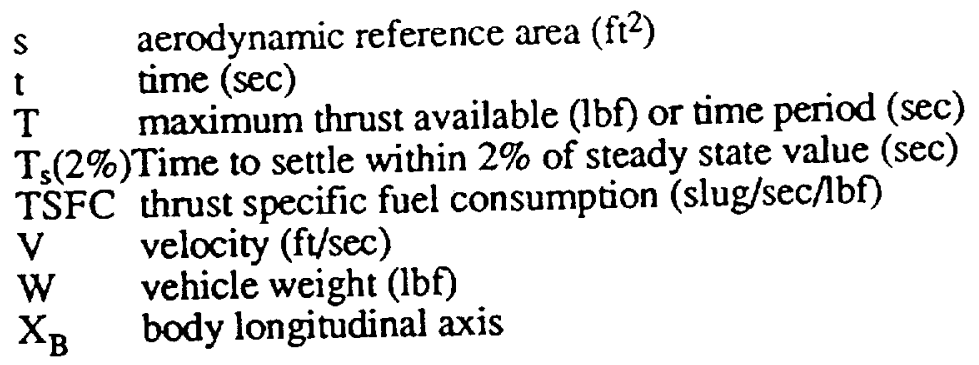

\section{Nondimensional Coefficients}

$\mathrm{C}_{\mathrm{d}_{0}} \quad$ zero lift drag component

$\mathrm{C}_{\mathrm{d}}$ drag coefficient

$\mathrm{C}_{\mathrm{l}} \quad$ lift coefficient

\section{Greek Symbols}

$\alpha \quad$ angle of attack (deg)

$\beta \quad$ sideslip angle (deg)

$\gamma \quad$ flight path angle (deg)

$\delta$ denotes perturbation parameter

$\partial$ partial differential operator

$\varepsilon \quad$ angle between thrust vector and body longitudinal axis (deg)or a perturbation parameter depending on the context

$\eta \quad$ throttle setting

$\theta \quad$ longitude (deg)

$\lambda$ general costate variable

$\mu$ gravitational constant for the Earth (product of Earth's mass and

universal gravitational constant, units are lbf $\mathrm{ft} 2 / \mathrm{slug}$ )

$\zeta \quad$ damping ratio

$\rho$ atmospheric density (slugs/ $/ \mathrm{ft}^{3}$ )

$\sigma \quad$ bank angle (deg)

$\tau \quad$ stretched time in boundary layer, $-t / \varepsilon$

$\phi \quad$ latitude (deg)

$\Psi \quad$ heading angle (deg)

$\omega$ rotational velocity of the Earth ( $\mathrm{rad} / \mathrm{sec})$

$\omega_{n}$ natural frequency ( $\mathrm{rad} / \mathrm{sec}$ )

$\omega_{\mathrm{d}}$ damped natural frequency $(\mathrm{rad} / \mathrm{sec})$ 


\section{Subscripts}

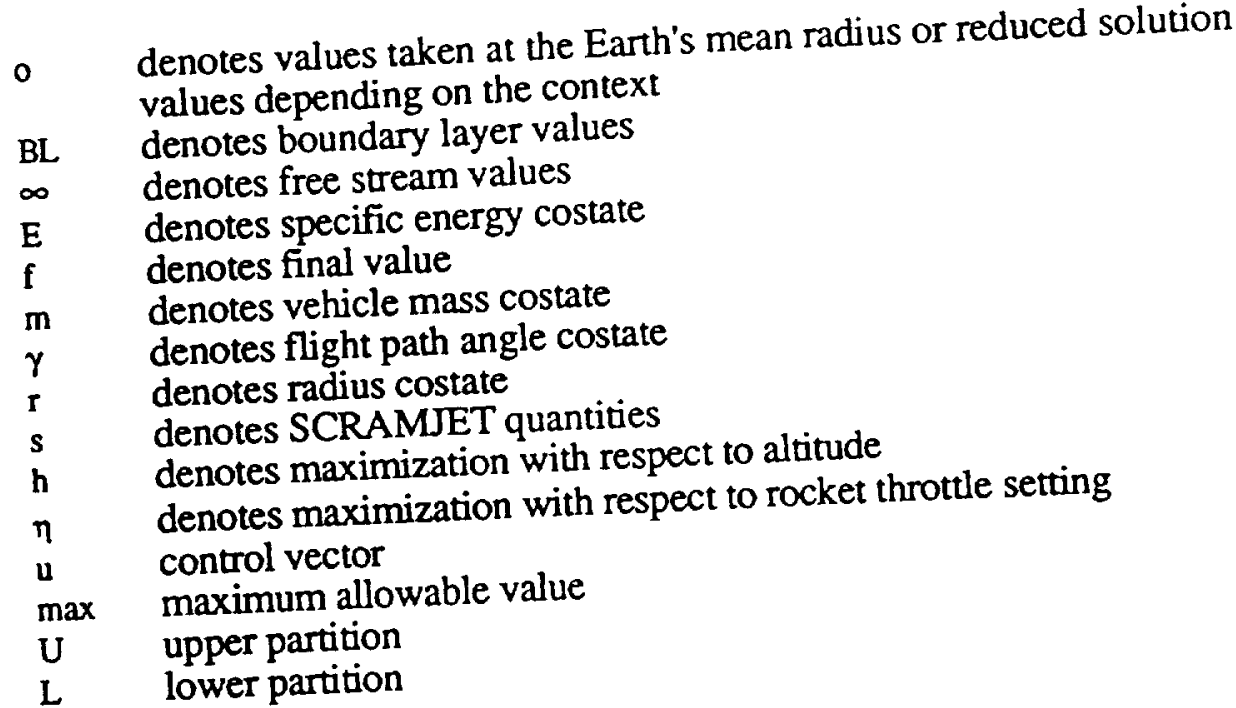

\section{Superscripts}

denotes differentiation with respect to time $t$

- denotes differentiation with respect to time $\tau=t / \varepsilon$

- denotes optimal value for given energy level or Hamiltonian function in which control has been eliminated using necessary conditions for optimality

T denotes matrix transpose operation

$\rightarrow$ denotes vector quantity

\section{Constants and Parameter Values}

$\begin{array}{ll}\mathrm{s} & 3780.0 \mathrm{sq} . \mathrm{ft} . \\ \mathrm{l} & 150 \mathrm{ft} . \\ \mathrm{K} & 0.1626 \\ \mathrm{C}_{\text {do }} & 0.0215 \\ \mathrm{r}_{\mathrm{o}} & 3959 \mathrm{miles}(6.370949 \mathrm{e} 06 \mathrm{~meters}) \\ \mu & 1.4094819 \mathrm{e} 16(\mathrm{lbf} \mathrm{ft} 2 / \mathrm{slug}) \\ \mathrm{go} & 32.2 \mathrm{ft} / \mathrm{sec} 2(9.8 \mathrm{~m} / \mathrm{sec}) \\ \omega & 7.27 \mathrm{e}-05 \mathrm{rad} / \mathrm{sec}\end{array}$




\section{Acronyms and Abbreviations}

CFD Computational Fluid Dynamics

DARPA Defense Advanced Research Projects Agency

GHAME Generic Hypersonic Aerodynamic Model Example

GN\&C Guidance, Navigation, and Control

NASA National Aeronautics and Space Administration

NASP National Aerospace Plane

NLT Lift Control Solution resulting from Nonlinear Transformation

SCRAMJET Supersonic Combustion Ramjet

SP1 Approximate Boundary Layer Lift Control Solution

SP2 Linearized Boundary Layer Lift Control Solution

TPBVP Two Point Boundary Value Problem 


\section{Summary of Work Completed}

The work completed to date is comprised of the following: a simple vehicle model representative of the aerospace plane concept in the hypersonic flight regime, fuel-optimal climb profiles for the unconstrained and dynamic pressure constrained cases generated using a reduced order dynamic model, an analytic switching condition for transition to rocket powered flight as orbital velocity is approached, simple feedback guidance laws for both the unconstrained and dynamic pressure constrained cases derived via singular perturbation theory and a nonlinear transformation technique, and numerical simulation results for ascent to orbit in the dynamic pressure constrained case.

A hypersonic research airplane concept studied by NASA in the mid-1970's has been selected as a nominal vehicle configuration. A variety of windtunnel data is available for this vehicle in the open literature over the Mach range 0.2 to 6.0. A full-scale vehicle of 150 feet total length and 200,000 pounds gross take-off weight representative of the $\mathrm{X}-30$ research aircraft is assumed. The vehicle model consists of separate modules for the estimation of aerodynamic and propulsive forces. The aerodynamic model, currently restricted to the hypersonic regime, is based on coefficients obtained by curve fits to windtunnel data at Mach 6.0. A parabolic drag polar is assumed and the induced drag coefficient is assumed independent of Mach number via the Mach Independence Principle. A largely analytic aerodynamic model based on a combination of Newtonian flow and blast wave theories was also investigated. The propulsion system is assumed to consist of a bank of six SCRAMJET engine modules that operate continuously at stoichiometric conditions when above Mach 10 and a rocket engine rated at 15,000 pounds gross thrust that can be turned on or off as dictated by optimality conditions. A simple conceptual SCRAMJET model, largely analytic and well suited for trajectory optimization, has been developed and is outlined in this report.

The mission of single-stage ascent to orbit was considered, and fuel-optimal ascent trajectories were generated numerically using a reduced order dynamic model. This moriel 
results from the following: application of an energy-state approximation, the assumption of a non-rotating Earth, the assumption that flight is constrained to a vertical plane, and finally by imposing time scale separation of altitude and flight path angle dynamics from energy and mass dynamics via singular perturbation theory. Rocket throttle control as currently modeled appears linearly in the Hamiltonian. The possibility of a singular arc in rocket throttle setting was examined and was shown to be non-optimal. Thus, the optimal control for rocket propulsion is bang-bang. An analytic condition for switching rocket thrust on, as orbital velocity is approached, was derived and is presented in this report. A zeroth-order boundary layer solution which accounts for altitude and flight path angle dynamics was formulated. A simple costate approximation was adopted to allow implementation but resulted in an unstable feedback guidance law. An improved costate estimate was subsequently formed by linearizing the boundary layer necessary conditions about the reduced solution. The unstable modes were then successfully suppressed. The resulting stable nonlinear feedback guidance law applies to the unconstrained case. The incorporation of a dynamic pressure constraint leads to difficulties in generating a boundary layer solution. A suboptimal lift control solution was derived via a nonlinear transformation technique as an alternative approach while the afore mentioned case is being investigated.

A numerical simulation of the hypersonic phase of fuel-optimal ascent-to-orbit was carried out using the suboptimal guidance law mentioned above for the dynamic pressure constrained case and is documented in Section 6 of this report. Lastly, future research objectives are recommended. These include: theoretical investigation of the state constrained boundary layer problem, extension of the vehicle model to the subsonic and supersonic flight regimes, the modeling of SCRAMJET thrust dependence on angle of attack, modeling of the component of thrust that contributes to vehicle lift, and investigation of optimality conditions associated with discrete variations in SCRAMJET thrust. Also recommended is the consideration of additional constraints (temperature, acceleration and lift limits) and examination of three-dimensional maneuvers as may be required for plane change, lift modulation, or mission abort. 


\section{Introduction}

\section{The Space Transportation Problem}

Recent studies $[1,2]$ which review the state of space transportation warn that a cheaper, more reliable means for transporting both people and cargo to and from earth orbit must be developed in the next 20 years if the United States is to maintain its position as a world leader amongst space-faring nations. The current U.S. Space Transportation System (Space Shuttle Fleet) represents an effort to build one vehicle to serve many roles. Despite its technical success, it is unlikely that a future derivative of this vehicle can achieve the operational efficiency required to remain competitive in the growing international space launch business or enable the U.S. to open the so called "space frontier". Studies indicate that our projected transportation needs will best be served by a mix of expendable and reusable vehicles. Specifically the functions of cargo transport to orbit and two-way passenger transport should be separated. In the case of either mission we require fully reusable vehicles that are robust and reliable. Numerous configurations, fuels, propulsion methods, launch modes, and other characteristics have been studied to determine the more promising approaches. Two types of vehicles have emerged: vertically launched rocket vehicles of both one and two-stages and horizontal-take-off single-stage-to-orbit airbreathing vehicles. The latter are commonly referred to as aerospace planes [3]. These two competitors, advanced rocket and aerospace plane technologies, both promise drastic reduction in the cost of achieving orbit and must be developed now if we are to be ready to exploit the opportunities of space in the $21^{\text {st }}$ century. In this report, the problems associated with the guidance and control of the aerospace plane configuration are examined in light of its desired operational objectives. Then, the progress made in deriving guidance laws suitable for implementation on-board such a vehicle is reported.

\section{The Promise of NASP Technology}

The aerospace plane concept involves winged or all-body vehicles, fueled by liquid hydrogen, that can depart and land horizontally from conventional jet runways. The configuration of principal interest would be capable of flying to low-earth orbit using only a single stage. The critical technologies that must be advanced include air-breathing 
supersonic combustion ramjet (SCRAMJET) engines, high temperature materials, and hypersonic configuration aerodynamics $[1,4]$. This technology, if developed, would aid not only transportation to low earth orbit, but also a host of other potential hypersonic missions, both military and civilian, identified in various government sponsored studies over the past several decades. Configurations could range from advanced interceptors and high performance reconnaissance aircraft to transports capable of cruise at Mach 6-12[4-7]. A successful aerospace plane, configured for ascent to low-Earth-orbit, would not only achieve a payload mass fraction an order of magnitude greater than current rocket systems, but would also do the following: eliminate conventional rocket staging, offer on-demand assured launch for space rescue and national security, provide greater basing flexibility through operations from conventional airfields and self-ferry flight, and improve system survivability by eliminating our reliance on just two U.S. launch complexes. Such a vehicle could also provide improved mission safety through multi-engine redundancy, aircraft-type control, abort capability, and alternate mission-recovery paths (cross-range flight capability). Airplane-like operations would greatly reduce the large number of ground support personnel and eliminate vertical-assembly buildings, launch pads, special recovery flight operations, solid boosters, external tanks, and other logistics burdens that characterize the Shuttle. In short aerospace plane technology, when mature, could offer efficient, reliable and economic access to orbit [8].

\section{The History of the NASP Program and Its Program Objectives}

The aerospace plane concept, which dates back to the 1950's, was seriously investigated in the United States during the 1960's. Development was abandoned at that time due to technical barriers. Subsequently, pure rocket technologies began to dominate our research efforts. All but a few research programs in SCRAMJET propulsion at NASA Langley and with the Navy were terminated. In 1982 the Defense Advanced Research Projects Agency (DARPA) again began investigating the limits of air-breathing propulsion, aerodynamics, materials, and structures in an initiative termed Copper Canyon. In the period between 1982 and 1985, DARPA redefined the aerospace plane concept with laboratory support from NASA, the Air Force, and the Navy. By 1985 it was determined that advances in aerodynamics, structures and air-breathing hypersonic propulsion had significantly lowered the technological barriers encountered earlier, and a decision was made to initiate a technology development program $[4,7,9]$. This program is referred to as the National Aerospace Plane (NASP) Program, and features wide participation by 
Government agencies and industry. It targets the maturation of key technologies, and plans the feasibility demonstration of a radically advanced engine. The preliminary design of the airframe needed for an experimental flight research vehicle analogous to past X-1 and X-15 research aircraft is underway. The new engine will be built and ground-tested up to about Mach 8, while the new structures and materials needed to fabricate such a vehicle are being developed and tested. Since no ground test capability is available to simulate full scale flight conditions at Mach numbers much above this, and indeed none seems feasible, the propulsion system and its integration into the airframe must be validated experimentally in flight. A research aircraft, dubbed the X-30, is scheduled to fly in 1994-95 in a test program that will demonstrate hypersonic cruise and acceleration into low-Earth orbit $[1,4]$. If the flight program confirms the feasibility of aerospace plane technology, definition and full-scale engineering development of next generation transport vehicles can be initiated using this vehicle concept.

\section{Guidance, Navigation and Control of the Aerospace Plane}

This research project addresses the problems associated with the guidance and control of vehicles that may evolve from the NASP program. These vehicles, although varied in size and detail according to the intended mission, will be of similar character where guidance, navigation and control (GN\&C) issues are concerned. This family of vehicles will be referred to as "the" aerospace plane throughout this report.

The GN\&C of the aerospace plane will ultimately include many aspects, including ascent to orbit, aero-assisted maneuvers, and the like. An essential component to achieving the transportation cost reductions mentioned earlier is that of drastically simplifying launch operations. In order to make possible an order of magnitude reduction in the cost per pound of payload placed in orbit, aerospace plane operations will have to approach those of current commercial airlines [1] . Current GN\&C technology, with its reliance on premission planning is inadequate to meet the challenge of automatic and adaptive trajectory control $[9,10]$. On-board guidance algorithms are needed to provide rapid airline-like operations and to respond to changing mission demands. This research effort seeks to develop computationally efficient and robust analytical and computer methods suitable for on-board flight trajectory optimization. Energy methods and singular perturbation theory, 
which have been successfully applied to similar problems for fighter aircraft $[11-14]$, are the principal tools to be applied in this endeavor.

\section{Review of the Document that Follows}

What follows is a report of the progress made toward this end during the period July 1 , 1987 to October 31,1988 . Section 2 of this report contains a description of the selected vehicle configuration and its intended mission. A hypersonic aerodynamic model and models for SCRAMJET and rocket propulsion systems are presented in Section 3. Section 4 addresses the issues of vehicle sizing and the selection of engine scaling parameters. Section 5 provides an introduction to the ascent-to-orbit guidance problem and, after a suitable set of dynamic equations are introduced, various assumptions which lead to a reduced order model are investigated. Next, singular perturbation theory is used to derive a means for approximating the minimum fuel climb path and to develop guidance laws in feedback form. A nonlinear transformation technique is also employed as an alternative to solving the boundary layer problem. The possibility of intermediate values of rocket throttle exists and an argument is presented to show that such settings are non-optimal. An analytic form for the rocket throttle switching condition is also presented. The approximate fueloptimal climb paths for the unconstrained and dynamic pressure constrained cases are presented in the text as the analysis is developed. A numerical simulation of the hypersonic phase of ascent-to-orbit using a derived guidance laws for the dynamic pressure constrained case is documented in Section 6. The conclusions for this effort and recommendations for future research are given in Section 7. Appendices, which further detail various technical issues, appear at the end of the report. 


\section{Vehicle and Mission Description}

The aerospace plane concept in general involves winged vehicles, fueled by liquid hydrogen, that can depart and land horizontally from conventional runways, maintain hypersonic cruise in the upper atmosphere for long durations and accelerate to orbital velocity. All-body aircraft have also been considered as candidate vehicles. It is not clear at this time which vehicle type will be most suitable. The high ignition speed of hydrogen fuel is required for supersonic combustion and this fuel's greater energy content is advantageous, but it also has a higher specific volume than conventional hydrocarbon fuels. The resulting high fuel volume requirement may dictate a blended wing-body or an all-body design. Since these issues are yet to be resolved, this research effort will assume a winged vehicle. Many potential missions have been identified for such vehicles [6]. The particular mission of single-stage ascent-to-orbit which promises, by the use of airbreathing hypersonic propulsion and greatly reduced launch operations, order of magnitude reductions in the cost of placing payloads in low-Earth orbit is especially attractive. The National Aerospace Plane Program seeks to develop a hypersonic research aircraft, designated the $\mathrm{X}-30$, which is capable of demonstrating the feasibility of the technology required to achieve such operations. The research aircraft will be designed to realize four primary goals during flight tests [15]:

- To simulate cruise for extended durations at speeds between Mach 5 and Mach 10 and altitudes well above 100,000 feet.

- To demonstrate operation into and out of ordinary airports, including environmental acceptability.

- To demonstrate flight into orbit from a runway, powered by air-breathing engines and carrying several thousand pounds of payload.

- To establish rapid turnaround in space operations. (Considered by some even more important than maximizing the payload mass fraction to orbit.) 
Clearly the X-30, if successful, shall possess most if not all of the aerodynamic and propulsive characteristics of the general aerospace plane concept. Thus we shall consider a vehicle model representative of the $\mathrm{X}-30$ as suitable for investigating problems associated with the GN\&C of this family of vehicles. Unfortunately, the X-30 development program, currently in the technology demonstration phase, has been classified as secret by the U.S. Department of Defense. As a result, few details will be forthcoming in the open literature. This is not, however, a serious obstacle, since a number of hypersonic research vehicles of similar nature were designed in the 1970's and, accompanied by suitable windtunnel data, appear in the open literature.

A vehicle representative of the $\mathrm{X}-30$ has been selected for this project from the series of hypersonic vehicle configurations studied by NASA in that time period. This aircraft, for which three-dimensional windtunnel data are available over the Mach range 0.2 to 6.0 [1619], is a hypersonic research vehicle concept with a 70 degree swept delta planform. It features an airframe integrated SCRAMJET propulsion system. It was designed to be airlaunched from beneath the wing of a B-52 much the same as the earlier X-15. A photograph of a 0.021 scale model of this vehicle and the configuration's general dimensions, normalized to body length, are reproduced from reference [18] as Figures 1 and 2, respectively. Various configurations of this particular vehicle were tested. The configuration designated $\mathrm{B}_{1} \mathrm{~W}_{1 \mathrm{f}} \mathrm{V}_{\mathrm{l}} \mathrm{F}_{\mathrm{d}} \mathrm{E}$ was selected as the most appropriate for this study. Table 1 presents a summary of the selected configuration features.

Table 1 Summary of Vehicle Configuration Features

B 1 Body one, with a high profile nose reflecting a forward cockpit location

$W_{1 f}$ Wing one, $70^{\circ}$ swept delta planform with positive camber, forward location

$V_{t} \quad$ Vertical wing tip fins (as opposed to a center vertical tail), with $7.5^{\circ}$ toe-in

$F_{d} \quad$ Additional forward delta wing

E Underslung SCRAMJET engine modules 
ORIGINAL PAGE IS

OF POOR QUALITY

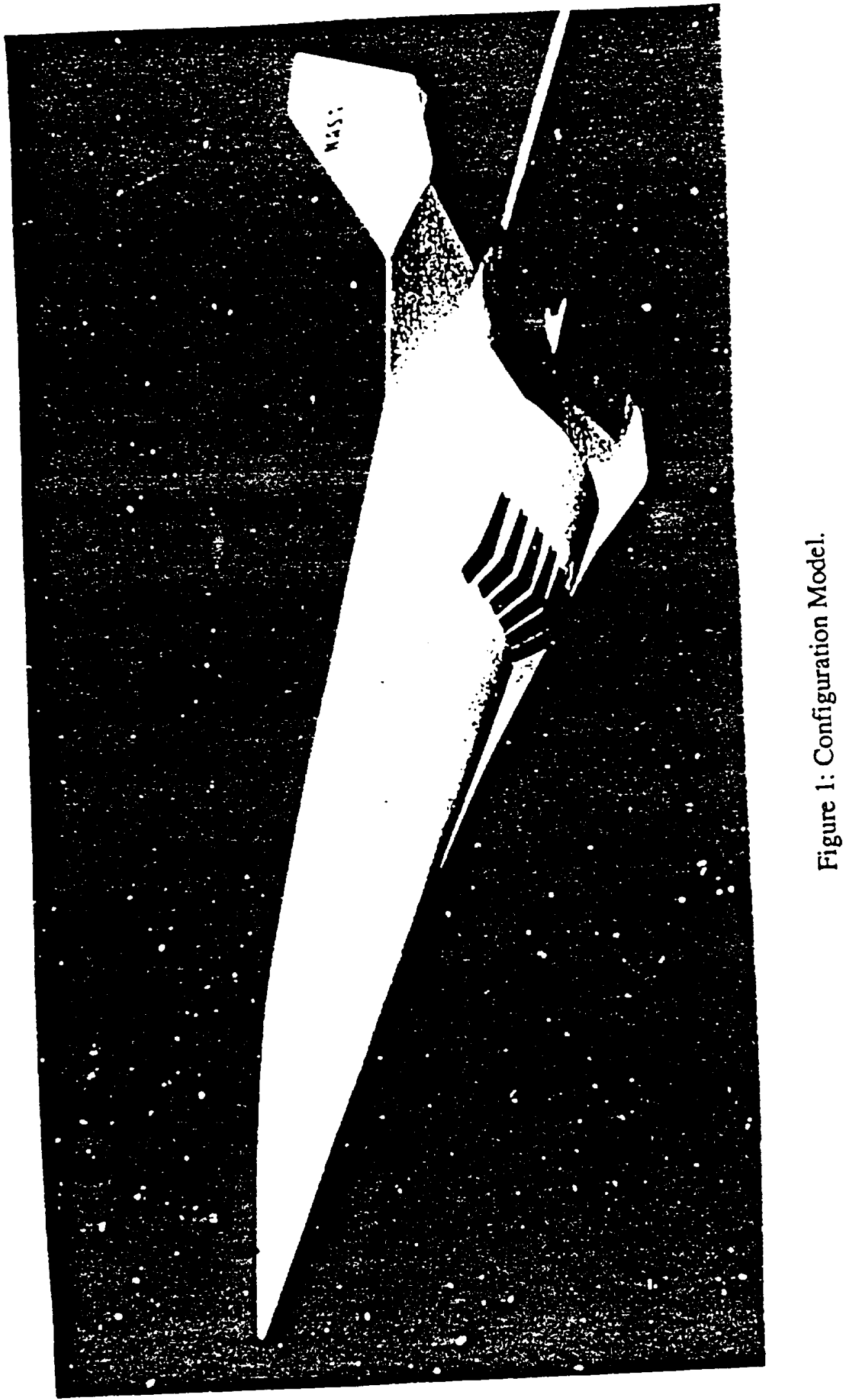


ORIGINAL PAGE IS

OF POOR QUALITY

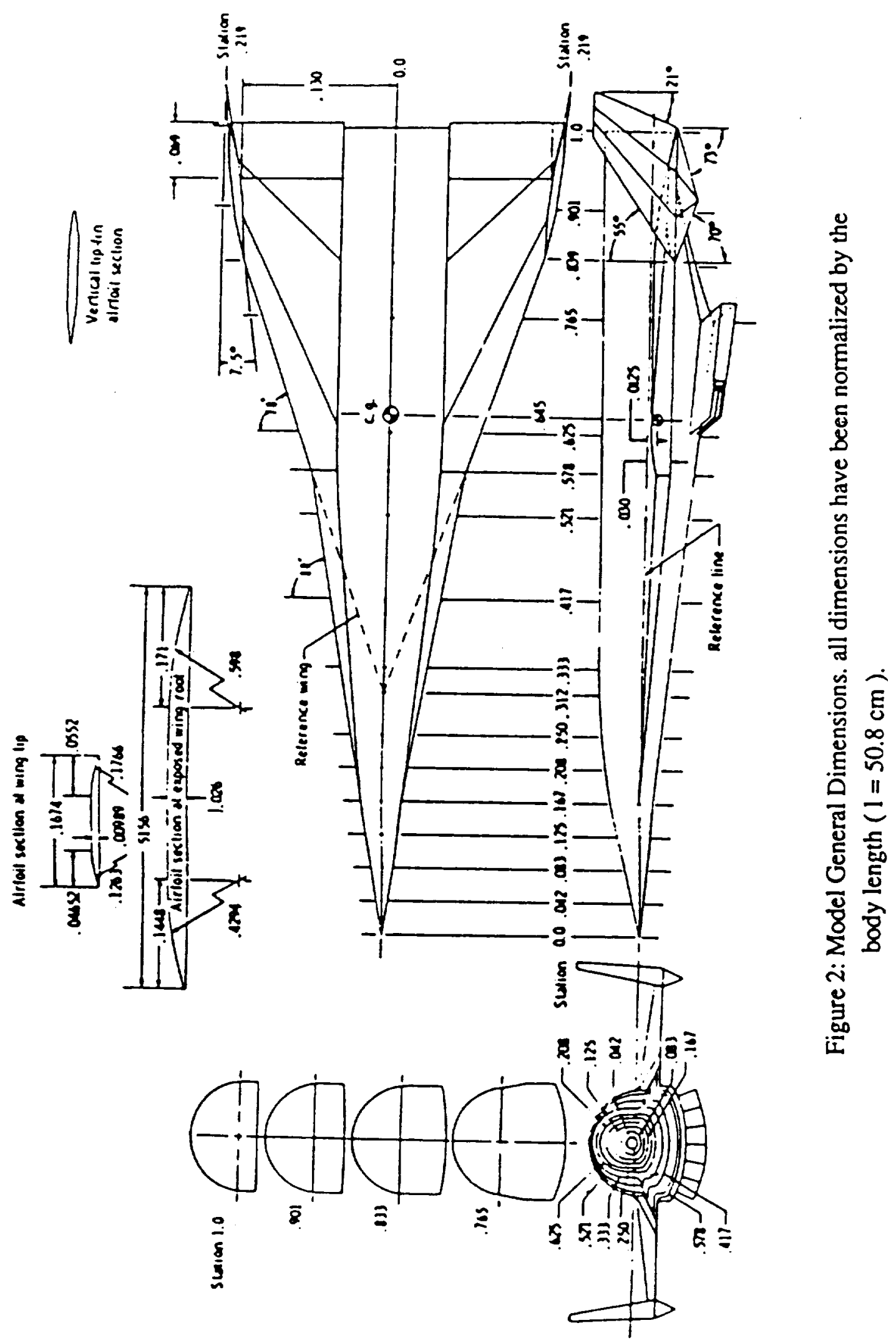


This design, based on a fixed geometry modularized SCRAMJET concept that is integrated closely with the basic airframe, is typical of the hypersonic air-breathing systems studied over the past two decades. The multiple engine modules are attached to a forebody precompression surface and exhaust over an aft body-nozzle surface. The inlets of the multiple rectangular SCRAMJET modules efficiently capture precompressed airflow contained between the vehicle and the forebody shock wave. The afterbody nozzle serves to increase SCRAMJET nozzle expansion area and allows the external SCRAMJET nacelle to be nearly stream aligned at the design Mach number for maximum installed thrust performance [20].

SCRAMJET engines, however, are incapable of operation below Mach 3 or 4 and suffer greatly reduced thrust capability at extreme altitudes. Aerothermodynamic constraints and the desire to achieve orbit will eventually force the vehicle to climb to such altitudes. Rocket propulsion is required for attitude control in space and re-entry. For these reasons the vehicle design must incorporate a multi-mode propulsion system. Candidate engine types include turbojets, ramjets, air-turbo-ramjets, SCRAMJETs, and rocket engines. One NASP Program objective is to achieve orbit using air-breathing propulsion alone, but many doubt the SCRAMJETs ability to power the vehicle all the way to Mach 25 [21]. Thus it is of interest to consider the use of a rocket engine prior to exiting the Earth's atmosphere. Which combination of engines is optimal and when to transition from one type to another are key questions to be answered. This report is focused on the hypersonic flight regime and addresses the question of SCRAMJET / rocket transition in a later section.

The above configuration, scaled to a length of 150 feet and weighing 200,000 pounds when fully fueled with liquid hydrogen, is used throughout the remainder of this report as a vehicle model representative of the $\mathrm{X}-30$ research aircraft [22]. The resulting reference area used for defining aerodynamic coefficients, (taken to be the projected area of the wing planform, including the part encompassed by the body) is 3780.0 square feet. In the next section, consideration is restricted to the hypersonic flight regime and a dual-mode propulsion system consisting of SCRAMJET and rocket engines is defined. Modeling of the vehicle's hypersonic aerodynamic characteristics and of the dual-mode propulsion system is discussed in detail. More about the sizing of the vehicle and its propulsion system is given in the section that follows. 


\section{Vehicle Modeling}

As detailed in the previous section, a hypersonic research airplane concept studied by NASA in the mid 1970's was selected as a nominal vehicle configuration. This design is based on a fixed geometry modularized SCRAMJET propulsion system that is closely integrated with the airframe. Consideration has been restricted to flight above Mach 5 and a dual-mode propulsion system (SCRAMJET / rocket) has been assumed. Vehicle sizing, discussed in the next section, results in a full scale vehicle of 150 feet total length and $200,000 \mathrm{lbs}$. gross take-off weight. For the purpose of trajectory optimization and simulation of derived guidance laws, a model reflecting the aerodynamic and propulsive characteristics of the vehicle is required. This model must be of a complexity commensurate with the expected accuracy of the performance analysis. In determining the nature of optimal flight profiles it is necessary that the models correctly predict the qualitative behavior of the aircraft, although to a limited degree, quantitative accuracy can be sacrificed for the sake of simplicity. In the study of hypersonic aircraft, a need currently exists for simple performance codes that use (to the extent possible) analytic aerodynamic and propulsion models and efficient optimization algorithms [7]. In this section the aerodynamic and propulsive models used to generate numerical results are presented. Included is the rationale for the models chosen and an indication of their shortcomings. Appendix A reviews the progress made in developing an analytic aerodynamic model for slender-bodied aircraft in the hypersonic flight regime.

\subsection{Aerodynamic Model}

A variety of windtunnel data are available for the selected vehicle configuration in the open literature over the Mach range 0.2 to 6.0 [19]. The aerodynamic reference area (s), taken to be the projected area of the wing planform, including the part encompassed by the body, is $3780 \mathrm{sq}$. $\mathrm{ft}$. The angle of attack along the optimal climb path will be shown to remain quite small, thus it is reasonable to assume a parabolic lift-drag polar of the form

$$
\mathrm{C}_{\mathrm{d}}=\mathrm{C}_{\mathrm{do}}+\mathrm{KC}_{\mathrm{l}}^{2}
$$


where $C_{D o}$ is given for trimmed flight at $M=6$ as 0.0215 and $K$ is estimated by a leastsquares curve fit to the data as 0.1626 [18]. The coefficient of the induced drag component, $\mathrm{K}$, is assumed independent of Mach number for $\mathrm{M}>5$ by means of the Mach Number Independence Principle [23, see also Appendix A]. Figures 3 and 4, produced from data available in [19], present the measured drag polar and the variation of lift coefficient with angle of attack for the trimmed vehicle at Mach 6.

This model, valid for the hypersonic flight regime, is deemed satisfactory for the present analysis. It cannot, however, account for important effects such as the variation in fuselage drag with changes in engine inlet geometry such as may occur in transitioning from ramjet to SCRAMJET propulsion. Nor does this model account for changes in drag due to vehicle trim requirements that change with center of gravity travel and with variations in the component of SCRAMJET thrust normal to the body longitudinal axis. The most serious shortcoming is the fact that the model is dependent on windtunnel data which is nonexistent when studying the trajectories of candidate vehicles in preliminary design. For these reasons, a simple analytic (to the extent possible) method for estimating the aerodynamic forces on a slender vehicle configuration in hypersonic flight is needed. A combination of Newtonian flow and blast wave theories yields such a model for some vehicle configurations in hypersonic flight and has been applied to the vehicle configuration detailed in this report. This work has not yet culminated in an aerodynamic performance prediction method suitable for use in trajectory optimization. Progress toward that end is reported in Appendix A. Methods suitable for modeling of aerodynamic characteristics in the subsonic and supersonic flight regimes are also under investigation.

\subsection{Propulsive Model}

Air-breathing propulsion systems now operate at flight Mach numbers up to 3 and at altitudes approaching 90,000 feet on a routine basis. There is a tremendous range of speed and altitude between these and orbital conditions over which air-breathing propulsion should be more efficient than rocket propulsion. This is due in large part to the fact that airbreathing systems draw oxygen from the atmosphere while rocket systems must carry their oxidizer along [24]. Aerospace plane technology under development today seeks to make possible vehicles capable of operating at sustained hypersonic speeds within the 


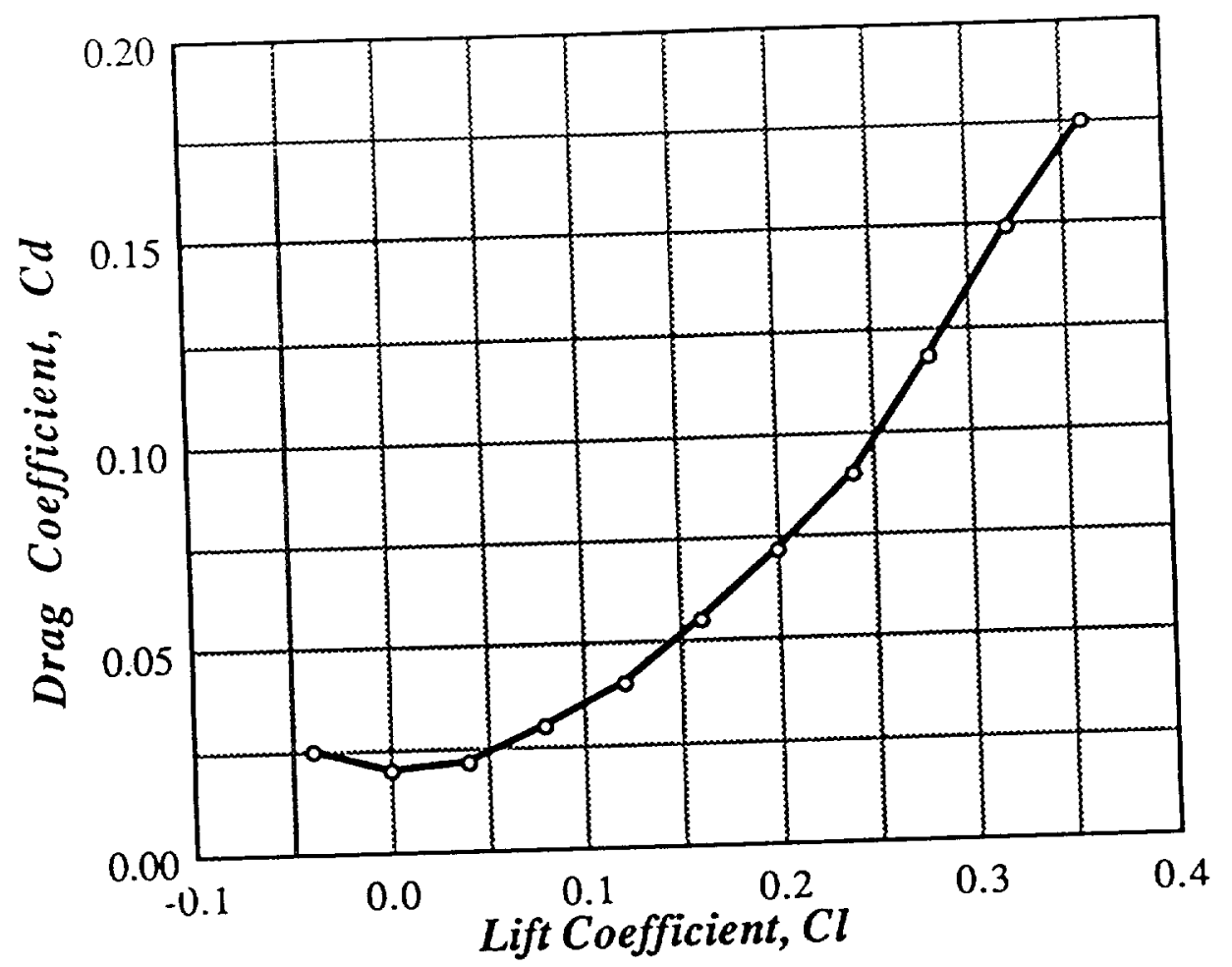

Figure 3. Drag Polar, experimental results for trimmed vehicle at Mach 6.

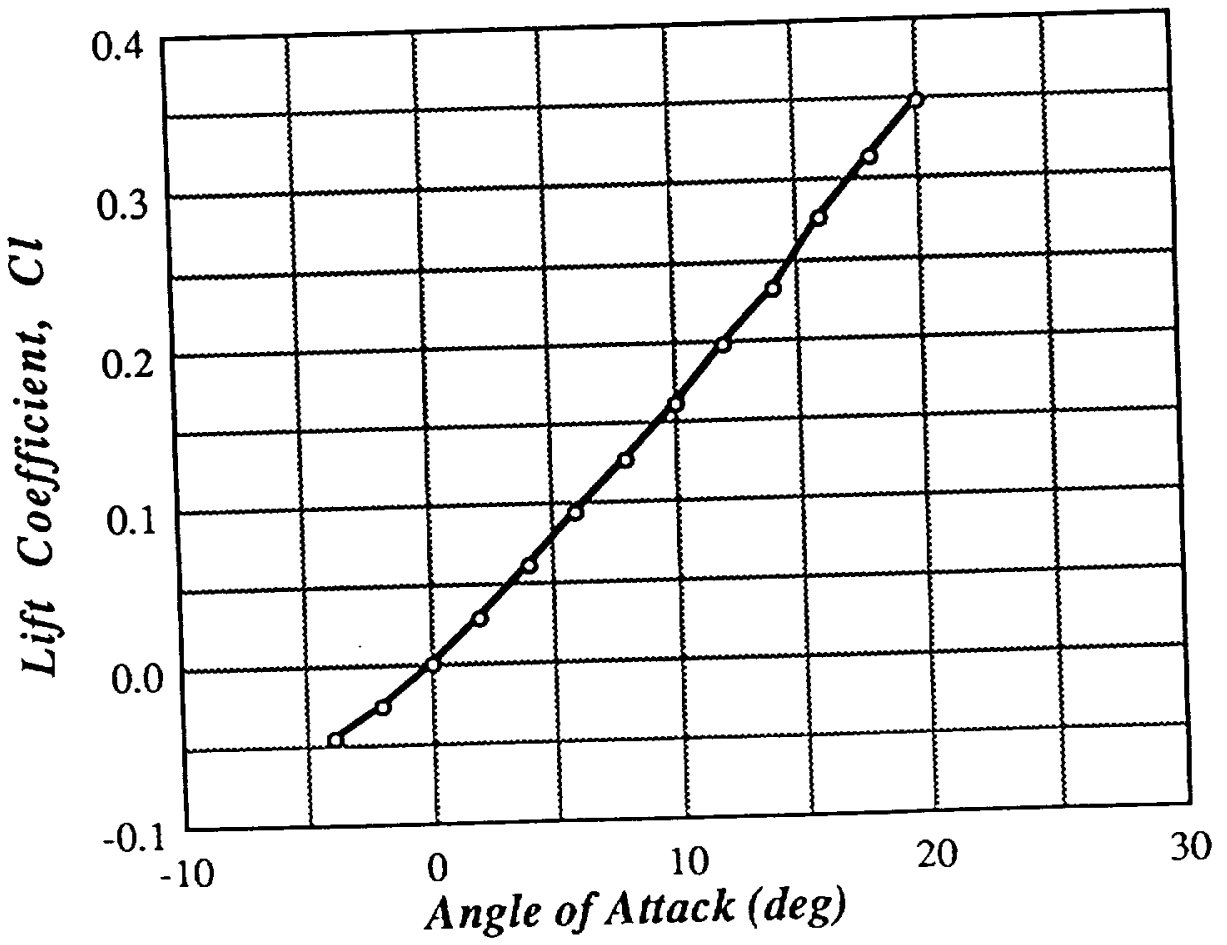

Figure 4. Measured variation of lift coefficient with angle of attack for trimmed vehicle at Mach 6 . 
atmosphere and/or operating as space launch vehicles for delivering payloads to orbit. Supersonic combustion ramjet (SCRAMJET) engine technology is the key to making such a vehicle concept a reality and when closely integrated with a host of various other advanced technologies may make possible a fully-reusable single-stage-to-orbit launch vehicle with a payload mass fraction an order of magnitude greater than that of current rocket systems [8].

Overall engine performance can be characterized by average specific impulse (Isp) which is defined as the number of units of thrust produced per unit of fuel weight flow rate. The units of Isp are seconds and the larger the value the more efficient the propulsion system. Rockets are limited typically to less than 500 seconds Isp, while a multi-mode aerospace plane propulsion system incorporating SCRAMJET engines is expected to average between 1,500 and 2,000 seconds Isp [8]. The potential performance gains are indicated in terms of specific impulse in Figure 5, which was reproduced from reference [6]. Clearly the ramjet and supersonic combustion ramjet (SCRAMJET), both airbreathers, can provide (as seen in Figure 5) efficient cruise propulsion for hypersonic vehicles. Note that the use of hydrogen rather than hydrocarbon (e.g. kerosene) fuels can improve engine performance at all flight speeds (see Figure 5). In particular, the liquid-hydrogen-fueled SCRAMJET offers the potential of Mach 7 performance comparable with that of hydrocarbon supersonic turbojet [6].

\subsubsection{SCRAMJET Model}

The supersonic combustion ramjet (SCRAMJET) has been well understood conceptually since the early 1960's but has been experimentally validated only in ground tests, and only up to Mach numbers of about 8 . To be attractive for orbital launch, its Mach number range must extend at least to 12 , and preferably beyond 15 [1]. The lack of appropriate unclassified experimental data, cycle analyses, and combustion analyses requires the use of a simple conceptual model for the purpose of vehicle trajectory optimization. What follows is a brief description of the model being used in this research effort and the philosophy behind it. 


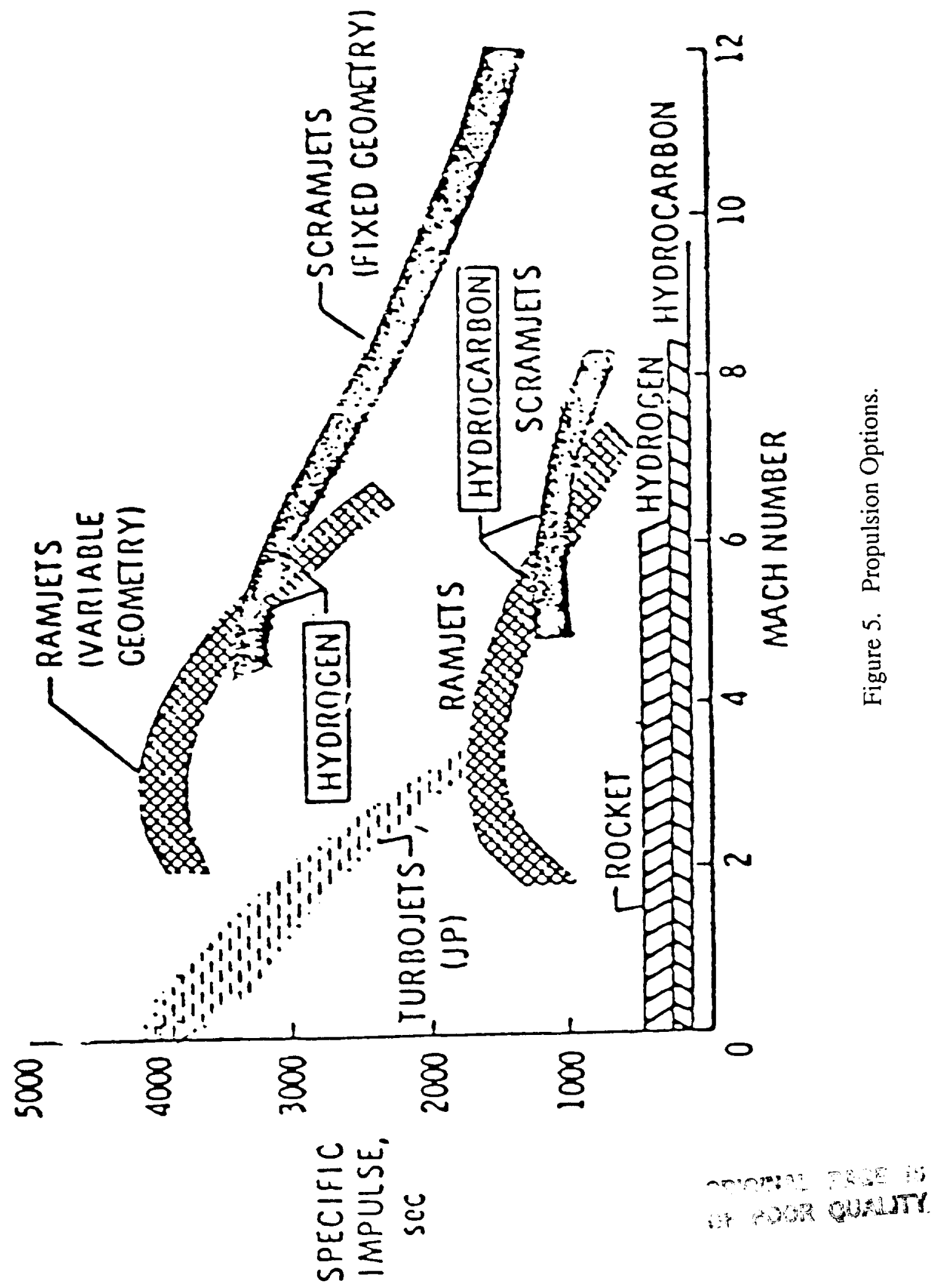


Conceptually, the SCRAMJET is as simple an airbreathing combustion device as one could imagine. In the case of the vehicle concept outlined in Section 2, the entire underside of the vehicle plays a role in the operation of the propulsion system. Figure 6 shows the basic configuration. Mechanically, the device can be thought of in terms of three elements. These are:

\section{Diffuser \\ 2. Combustor \\ 3. Expansion nozzle}

Hypersonic vehicle designers attempt to utilize the forward fuselage, strakes, and wings to provide the majority of the diffusion. The lower part of the three-dimensional oblique shock formed at the leading edges is tailored to the shape of the combustor inlet so that air enters at approximately Mach 3, but this depends on the flight speed. Combustion of hydrogen fuel takes place in the duct at supersonic speeds in order to minimize energy losses due to dissociation, which would be enormous if the more conventional subsonic ramjet cycle were to be used in high speed flight. Liquid hydrogen is the fuel of choice not only because of its high energy content, but because it can be made to burn in a supersonic flow due to its wide flammability limits and high flame speed. Finally, the combustion products are expanded through a nozzle, which, like the diffuser is designed into the contour of the lower fuselage.

As Figure 6 suggests, the propulsion system is mostly diffuser and nozzle. While these elements are fairly easy to model from the thermodynamic cycle point of view, the aerodynamics are quite complex, giving rise to a challenging design problem. Computational fluid dynamics (CFD) numerical techniques are being relied upon in conjunction with a new family of hypersonic test facilities to yield practical design solutions. Unfortunately, information on the current research is classified, so that realistic design data is not available for projects of this type.

The computational model used here to represent the SCRAMJET propulsion system was deliberately designed to be readily updated as new information becomes available. It directly accesses a standard atmosphere model (also easily adjustable to provide non- 


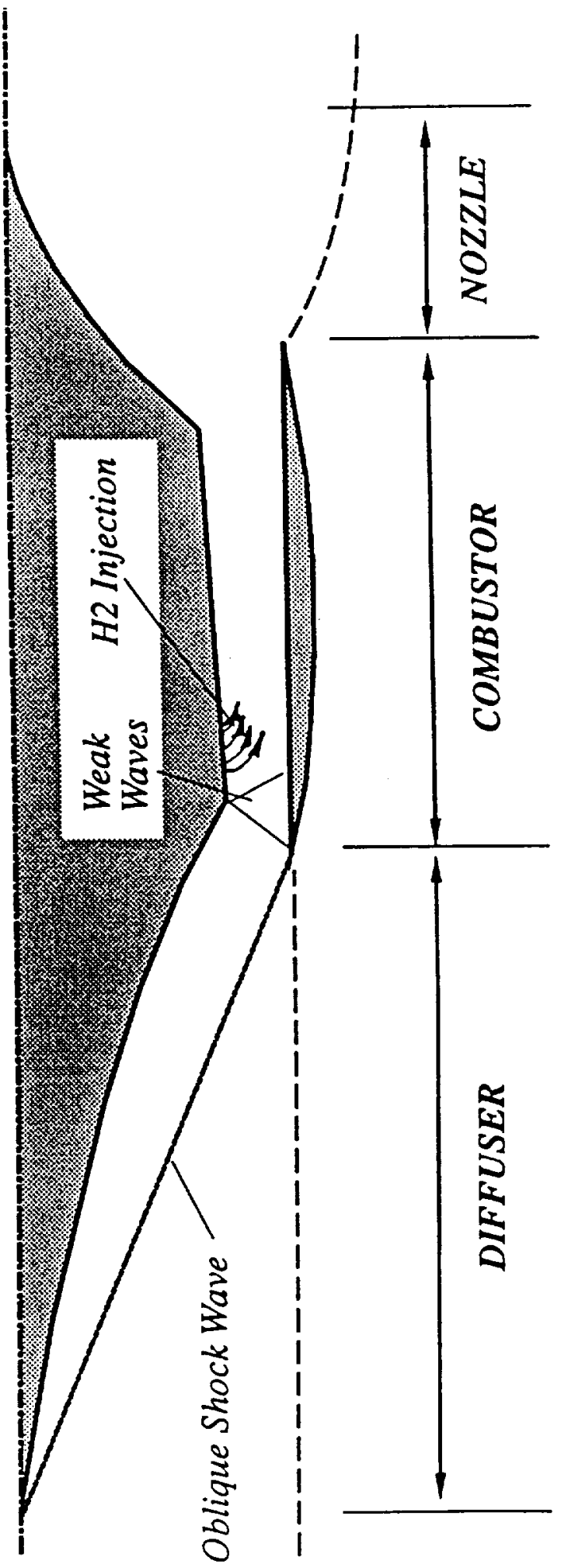

ह 
standard operating conditions), which simplifies its incorporation into a trajectory optimization program. The diffuser and nozzle performance is determined either with standard thermodynamic models or by means of optimal design curve fits such as those proposed by Billig [25]. Since information concerning recent progress in supersonic combustion was not available, a simple combustor model was incorporated. This is a straightforward Rayleigh line calculation. An iterative scheme is used to determine the nozzle entrance Mach number, by maintaining the mixture ratio at or below the stoichiometric value. No detailed combustion calculations with multi-species gases is attempted in the present version of the model although these could be readily incorporated as a more definitive model of practical SCRAMJET combustion comes into focus.

The propulsive drag estimate of Billig was incorporated to account in a simple way for some of the frictional losses. No attempt was made to incorporate vehicle integration effects in an interactive fashion. Experience with the aerodynamic simulation shows that very small vehicle attitude changes take place during equilibrium flight. Therefore in the present state of development, no vehicle attitude dependence has been included in the propulsion model. The flexibility of the algorithm will make such additions quite easy to make as the need for them is established. Fuel preheat due to its circulation as a coolant prior to combustion is currently ignored as is the addition of fuel into the combustor in excess of the stoichiometric ratio for the purpose of structural cooling. Another important effect not yet accounted for is the possibly large component of SCRAMJET thrust normal to the body longitudinal axis. This force, which contributes to overall vehicle lift and which can cause a large nose down pitching moment, may have a large impact on vehicle performance and should be properly modeled.

Figures 7-10 present calculated SCRAMJET performance as a function of Mach number at various altitudes in terms of the following quantities: fuel specific impulse, Isp, in units of seconds, thrust specific fuel consumption, TSFC, in units of pounds mass per hour per pound force, and net thrust, $T_{s}$, in pounds force. Results are for an engine module with a one square foot projected inlet area. Inlet area is the appropriate engine scaling parameter. The indicated performance is quite similar to that estimated by others $[26,27]$. Figure 10 presents the calculated fuel-to-air ratio, $f$, as a function of Mach number for several altitudes. This figure was included to illustrate the influence on thrust of constraining the fuel-to-air ratio to remain at or below its stoichiometric value. The resulting change in slope 
in the thrust curve that occurs as f reaches its stoichiometric value will later be shown to influence the unconstrained fuel-optimal climb path. The SCRAMJET computational algorithm also provides estimates of fuel flow rate, propulsion module thrust, propulsion drag, and the standard performance parameters. Additional references on SCRAMJET propulsion systems are included in the bibliography as [28-35].

\subsubsection{Rocket Model}

In addition to its SCRAMJET engines, the X-30 must carry rocket propulsion for attitude control in space and reentry. It is also of interest to consider the use of a rocket engine prior to exiting the Earth's atmosphere. The Pratt \& Witney RL10 rocket engine used on the Centuar upper stage is deemed suitable for this purpose $[21,36]$. The RL10 is rated at $15,000 \mathrm{lbf}$ thrust at $200,000 \mathrm{ft}$. with a nominal specific impulse of $444 \mathrm{sec}$. Propellants are liquid oxygen and liquid hydrogen with a nominal oxidizer-to-fuel ratio of 5:1. This engine, capable of multiple starts, has been tested in advanced versions that include variable thrust. The rocket model used in this study assumes the performance of the RL10 and makes a simple thrust correction below 200,000 ft. for losses due to atmospheric back pressure [37]. Thrust of the rocket is given by

$$
T_{r}=T_{\text {vacum }}-A_{E} p(h)
$$

where $A_{E}$ represents nozzle exit area and $p$ is atmospheric pressure given as a function of altitude. Figures 11 through 14 present predicted rocket performace as a function of altitude in terms of fuel specific impulse, thrust, thrust specific fuel consumption, and mass flow rate given a nozzle exit area of 1.0 square foot. 


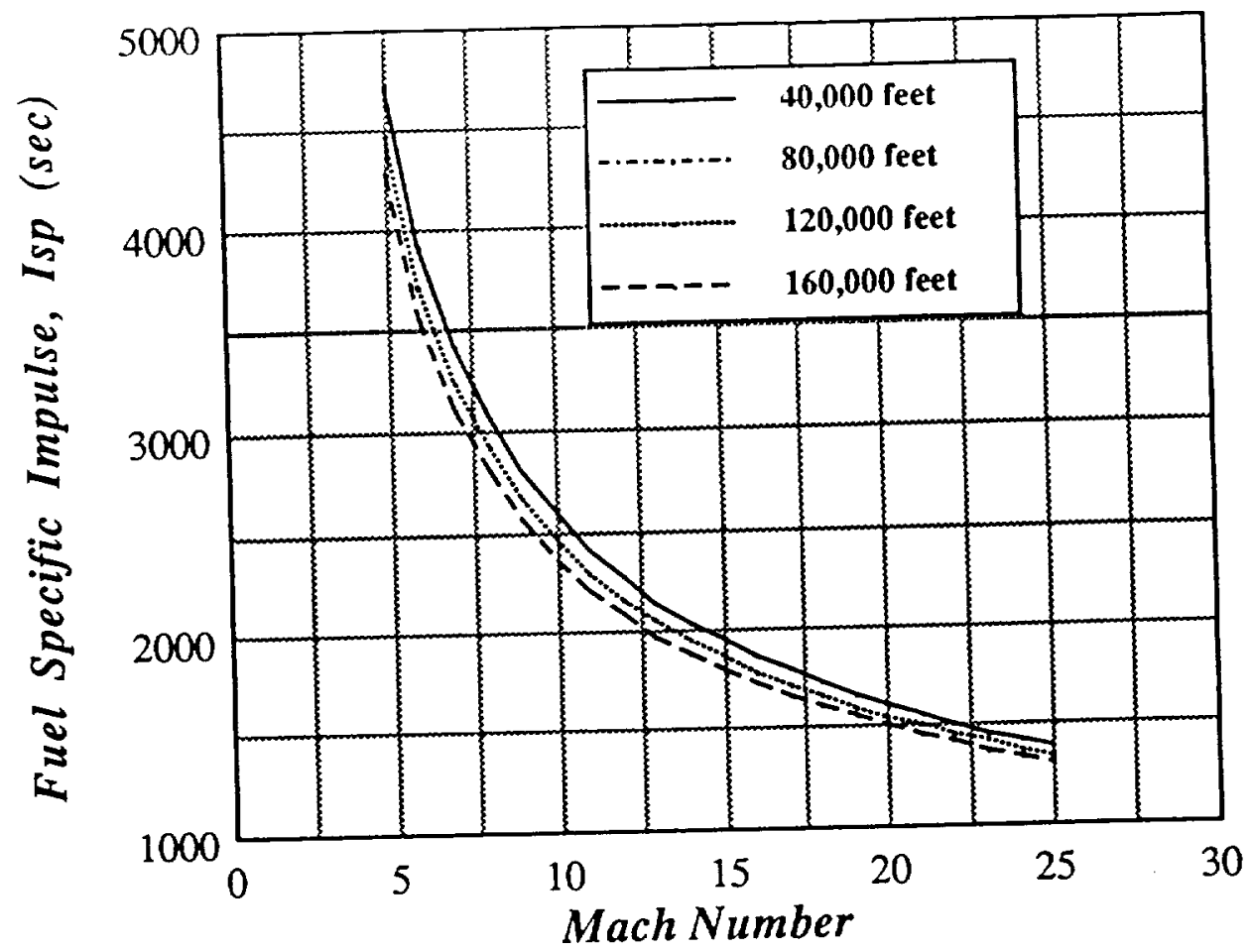

Figure 7. Predicted SCRAMJET Fuel Specific Impulse as a function of Mach number for various altitudes.

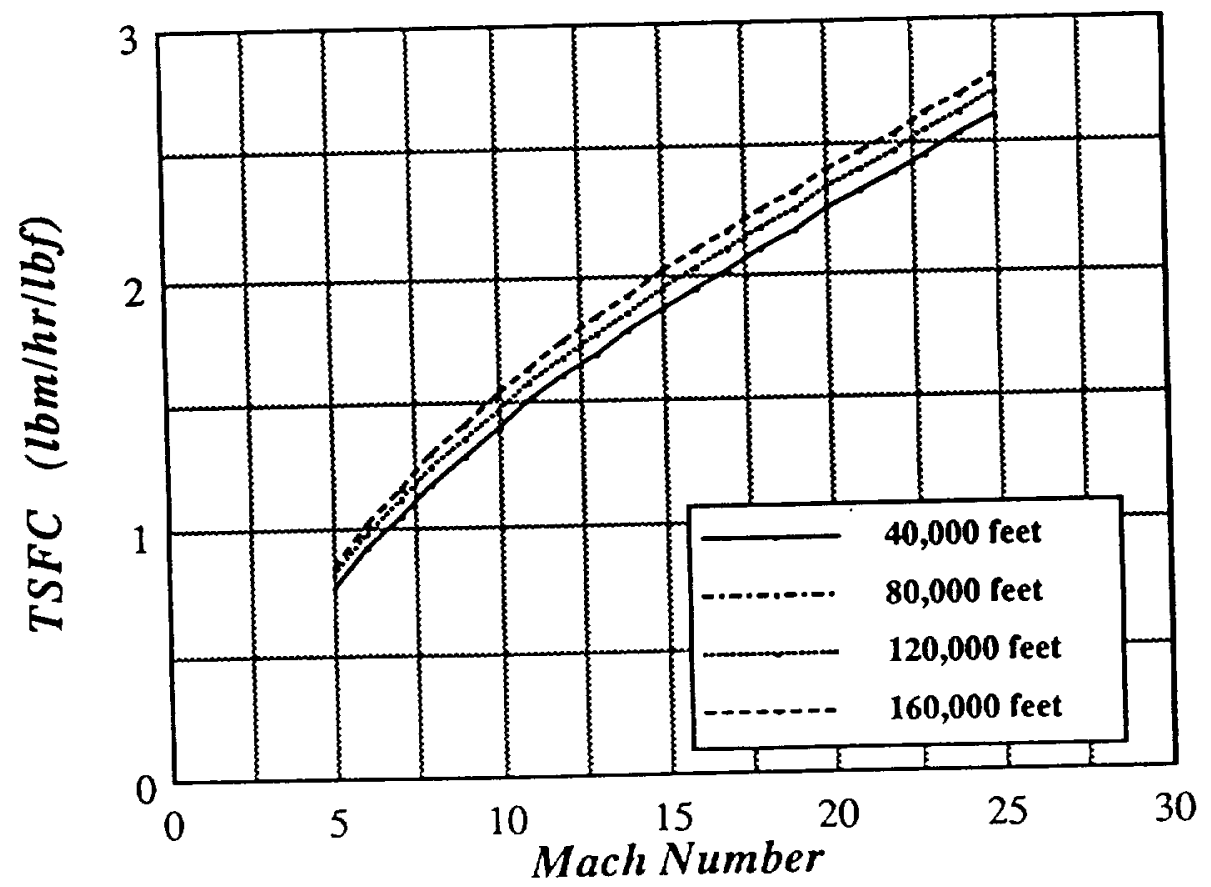

Figure 8. Predicted SCRAMJET Thrust Specific Fuel Consumption as a function of Mach number for various altitudes 


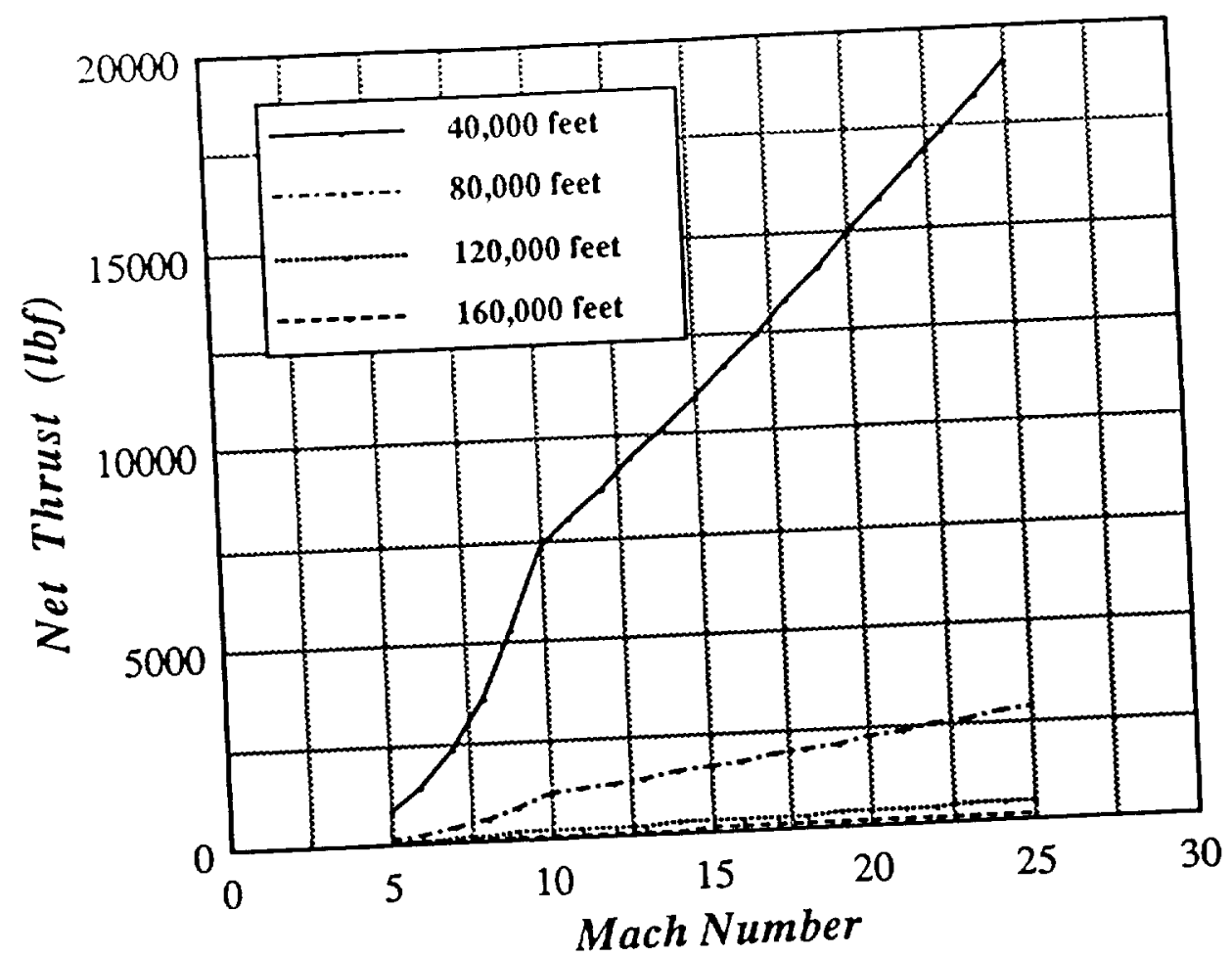

Figure 9. Predicted SCRAMJET Thrust as a function of Mach Number for various altitudes. Results are for a single engine module with a projected inlet area of 1 square foot.

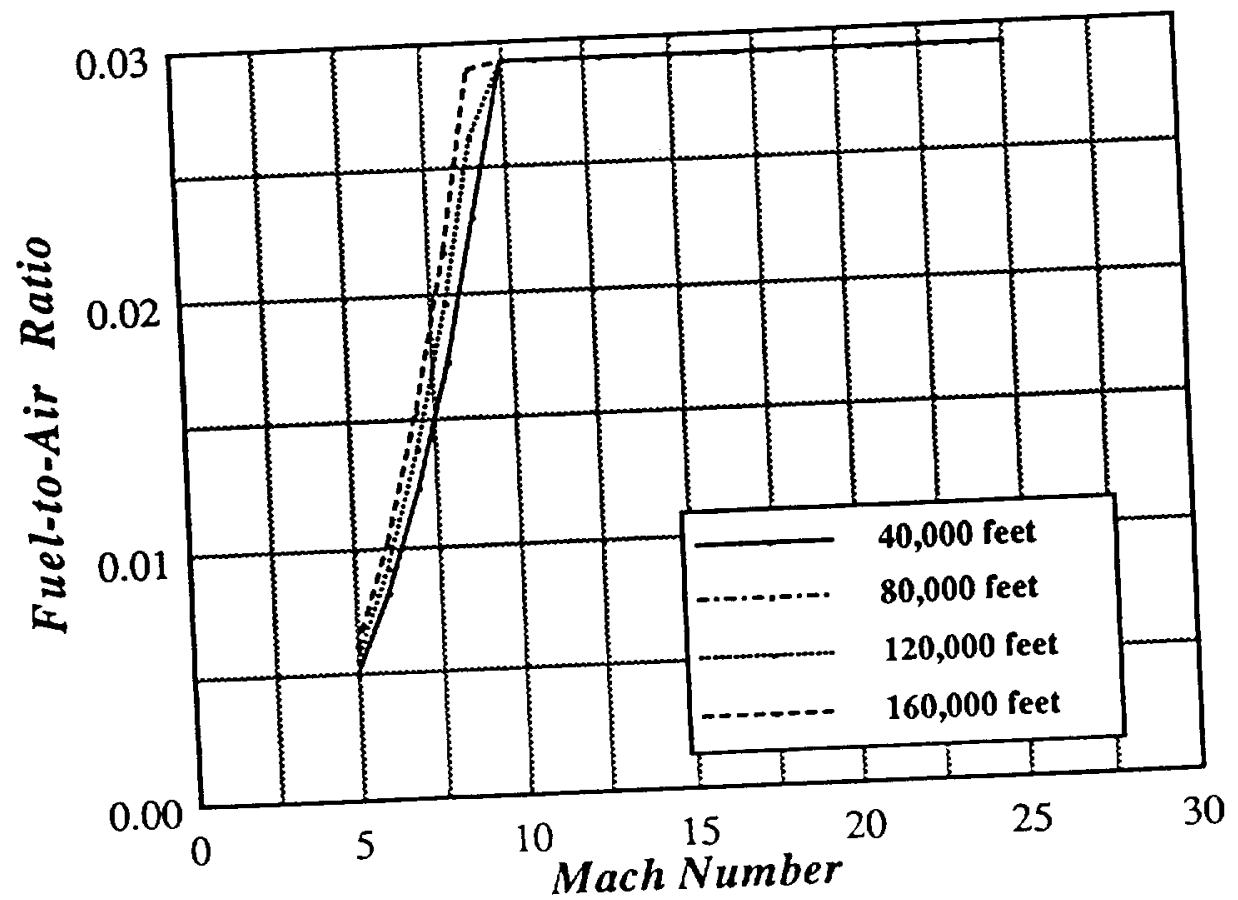

Figure 10. Predicted SCRAMJET Fuel-to-Air Ratio as a function of Mach number for various altitudes. 


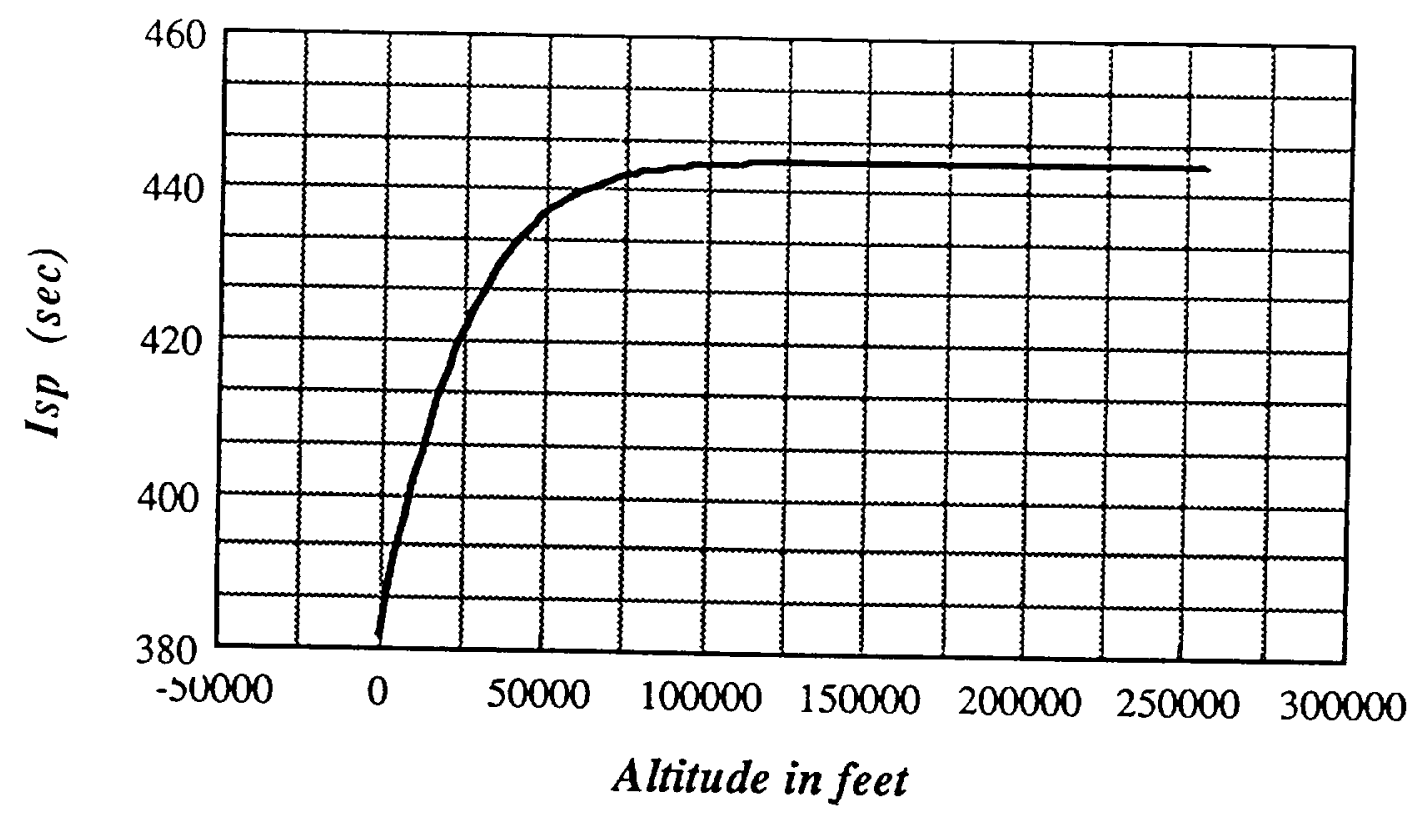

Figure 11. Predicted rocket performance, fuel specific impulse versus altitude, for a nozzle exit area of 1.0 square foot.

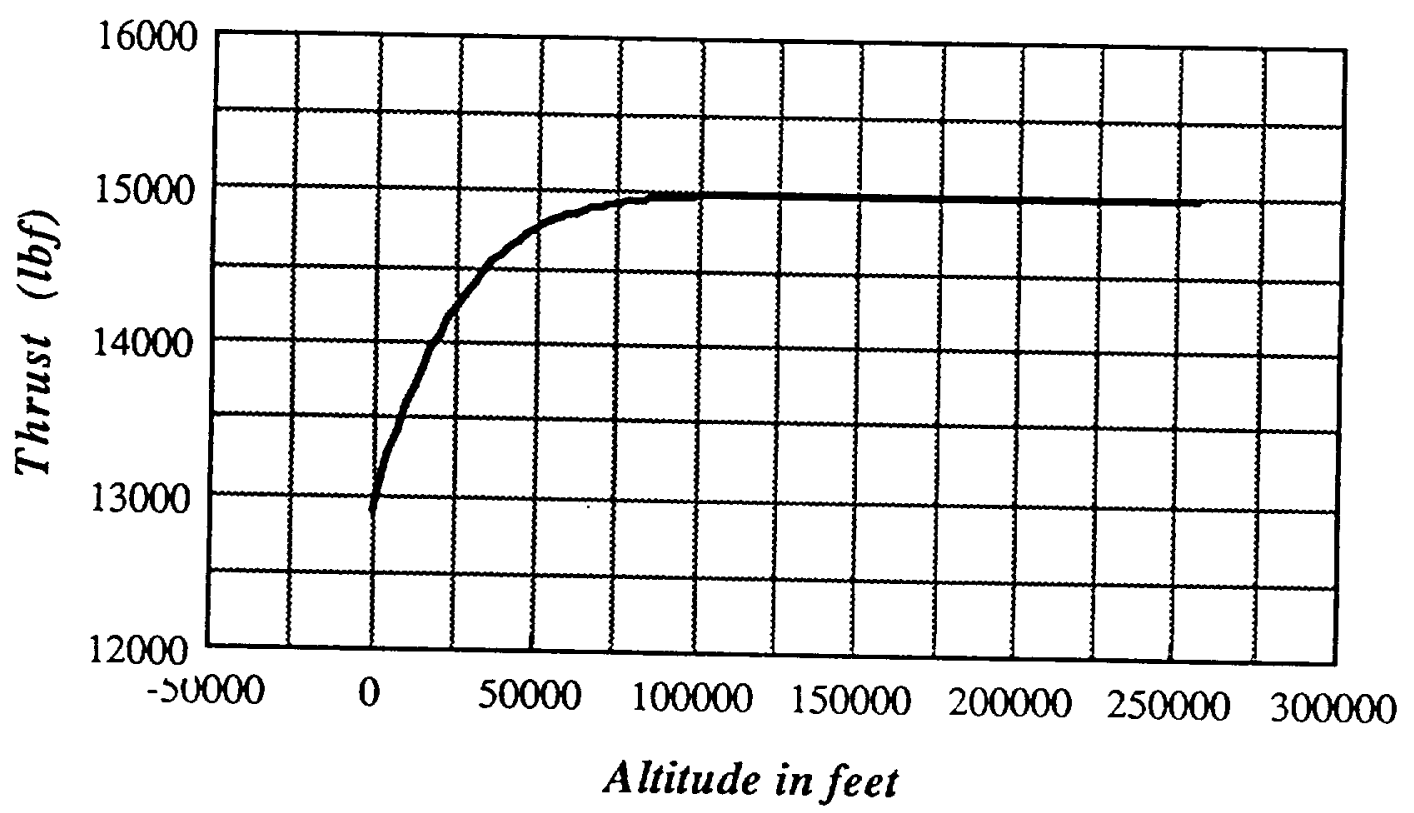

Figure 12. Predicted rocket performance, thrust versus altitude, for a nozzle exit area of 1.0 square foot. 


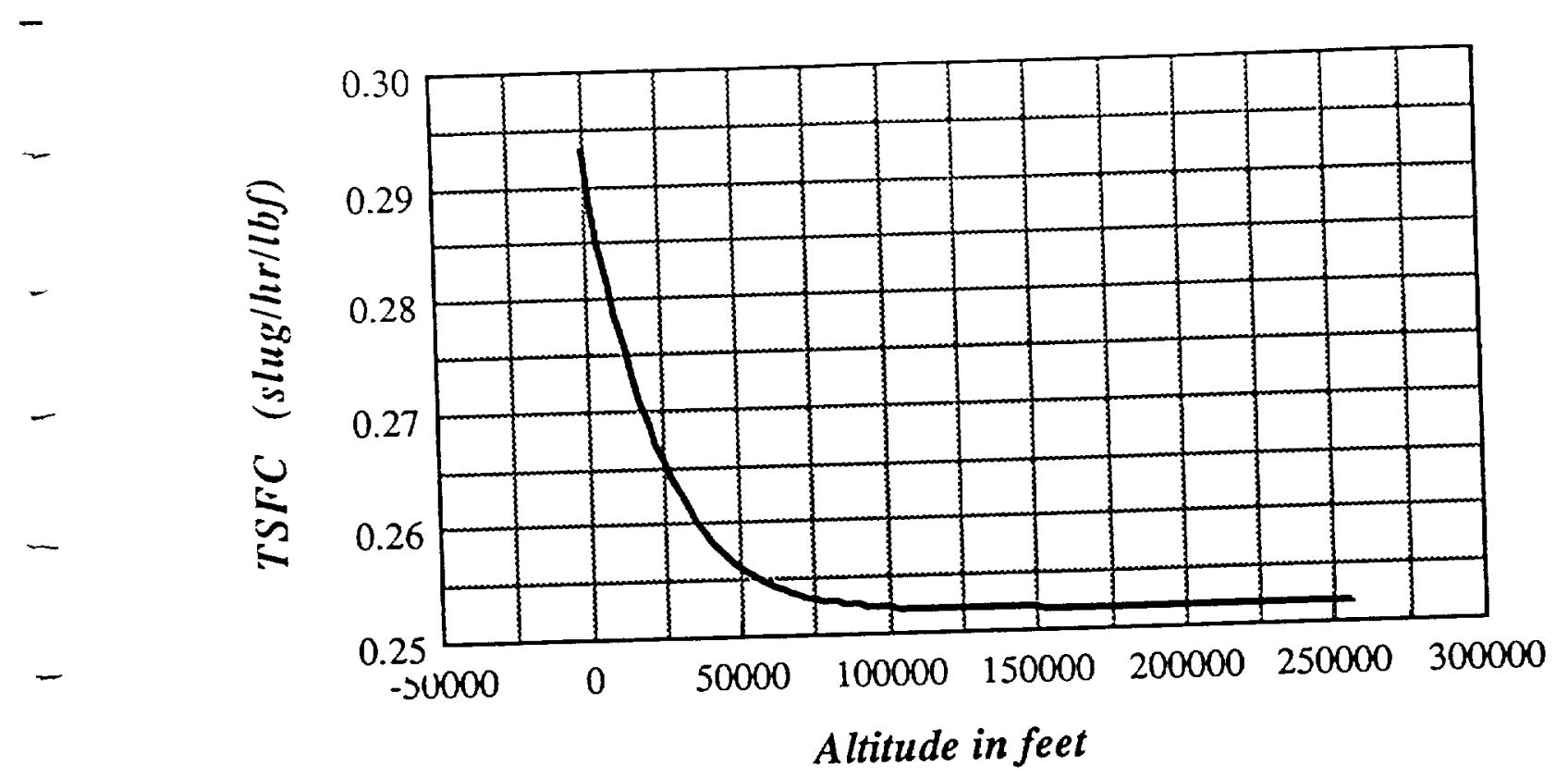

Figure 13. Predicted rocket performance, thrust specific fuel consumption versus altitude, for a nozzle exit area of 1.0 square feet

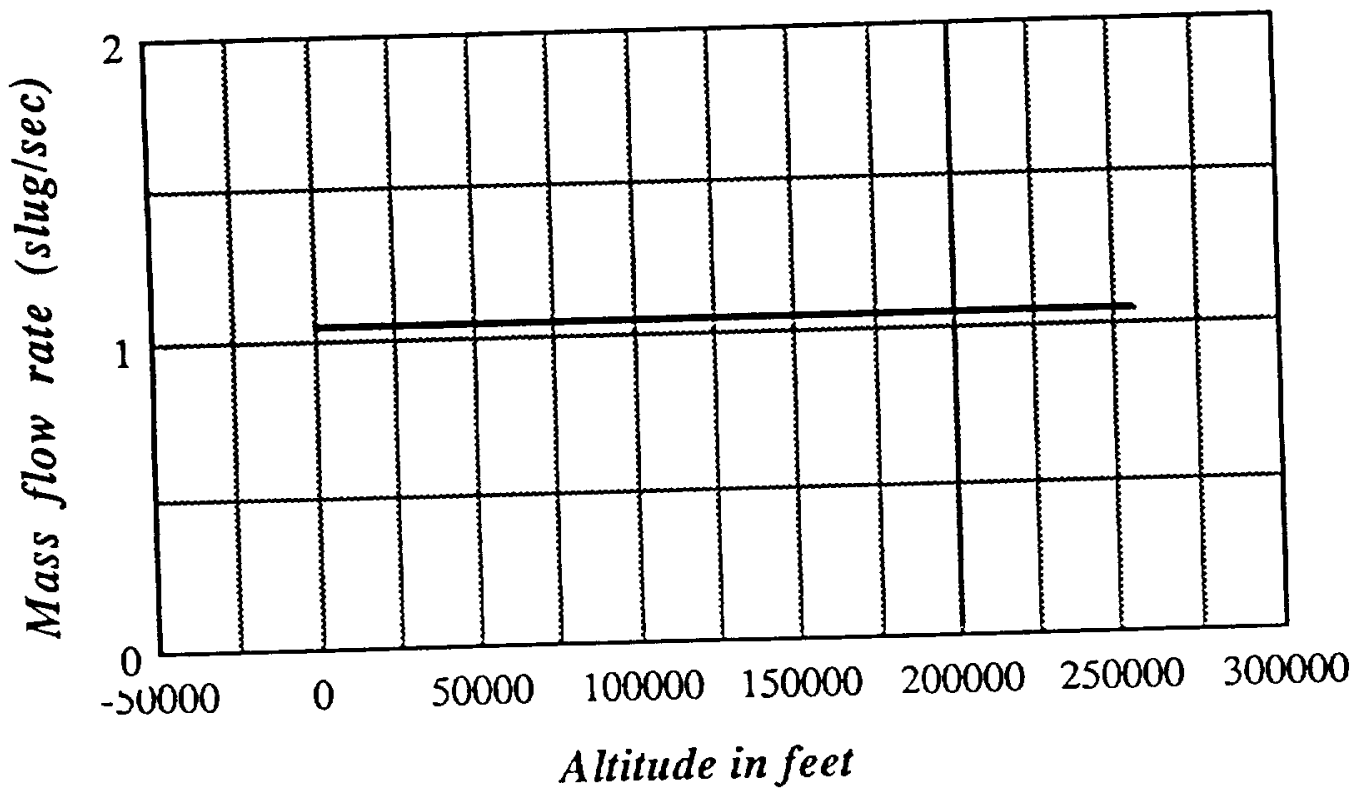

Figure 14. Predicted rocket performance, mass flow rate versus altitude, for a nozzle exit area of 1.0 square feet 


\section{Vehicle Sizing}

As previously detailed, a hypersonic research airplane concept studied by NASA in the mid-1970's was selected as a nominal vehicle configuration. This design is based on a fixed geometry modularized SCRAMJET propulsion system that is closely integrated with the airframe. Consideration has been restricted to flight above Mach 5 and a dual mode propulsion system (SCRAMJET / rocket) has been assumed. This vehicle has been sized to resemble the scale of the proposed X-30 research aircraft and results in a full scale vehicle of 150 feet total length and 200,000 lbs. gross take-off weight [38]. The resulting aerodynamic reference area (s), taken to be the projected area of the wing planform, including the part encompassed by the body, is 3780 square feet.

The hypersonic aerodynamic characteristics of this vehicle configuration were used to size the SCRAMJET engine inlet area while assuming no other type of propulsion system to be operating. Figure 15 presents the vehicle's calculated level flight envelope as a function of SCRAMJET engine inlet area assuming weight remains constant at the assumed take-off value of 200,000 pounds and that lift equals weight. The inner-most envelope corresponds to 360 square feet of inlet area (6 SCRAMJET modules at 60 square feet each) which provides sufficient thrust for cruise between Mach 8 and Mach 12. The inlet area required to cruise increases dramatically with Mach Number, approaching the requirement for 900 square feet as orbital velocity is approached. This case corresponds to the outermost envelope shown. This trend agrees with that indicated in reference [39]. Note that a maximum allowable dynamic pressure constraint ( $q=2000$ psf is shown in the figure) severely limits the accessible flight envelope and thus greatly constrains the ability to optimize the ascent trajectory. The inlet area required per engine module exceeds $100 \mathrm{sq}$. $\mathrm{ft}$. if the level flight envelope is to cover the Mach range from 5 to 20. This value is approximately 10 times the inlet area (note that inlet area refers to the cross sectional area of the combutor inlet) of the configuration shown in Fig. 2. Fuselage drag predictions based on the referenced windtunnel data have not been modified to reflect this change in vehicle frontal area and it has been assumed that the ram drag calculations made in computing SCRAMJET thrust account for the additional drag incurred. A more accurate analysis 
would necessarily have to correct the fuselage drag estimate for this change in configuration.

Figure 16 again presents the calculated level flight envelope as a function of inlet area given only SCRAMJET propulsion, but now it is assumed that lift equals weight minus centrifugal force. Weight is again held constant at its assumed take-off value of 200,000 pounds. This figure indicates that for the postulated vehicle configuation a narrow corridor is avaiable for ascent to low-Earth orbit using SCRAMJET propulsion alone. Figure 17 presents the equivalent SCRAMJET envelope but for weight assumed constant at 77,000 pounds. This weight corresponds to an estimate of the remaining vehicle mass upon achieving orbital velocity. Having accounted for the decreased vehicle weight we find that the afore mentioned corridor is widened.

Since rocket propulsion will be required for orbit circularization, on orbit maneuvers and initiation of reentry, it is of interest to examine the optimality of using this available rocket propulsion during ascent. Figure 18 presents the calculated level flight envelope for seperate SCRAMJET or rocket propulsion, and a combination of the two. The innermost envelope corresponds to a propulsion system consisting of 6 SCRAMJET engine modules with 100 square feet of projected inlet area per module. When compared to the curve corresponding to 6 SCRAMJET modules with 150 square feet of projected inlet area each, one sees that there is a level of total SCRAMJET inlet area (that is inlet area per module) between 100 and 150 below which orbit cannot be achieved. Increased engine inlet area comes with a tremendous drag penalty at the lower Mach numbers, thus it would appear that the use of rocket propulsion to agument SCRAMJET thrust would be advantageous to a point. A careful design trade-off in sizing the SCRAMJET and rocket engines, rather than simply sizing the rocket to perform its on-orbit duties, will produce the most efficient configuration. No such trade-off is attempted here. All of the numerical results that follow in this report were generated assuming 6 SCRAMJET modules with 150 square feet of projected inlet area per module and a rocket rated at 15,000 pounds thrust at 200,000 feet. 


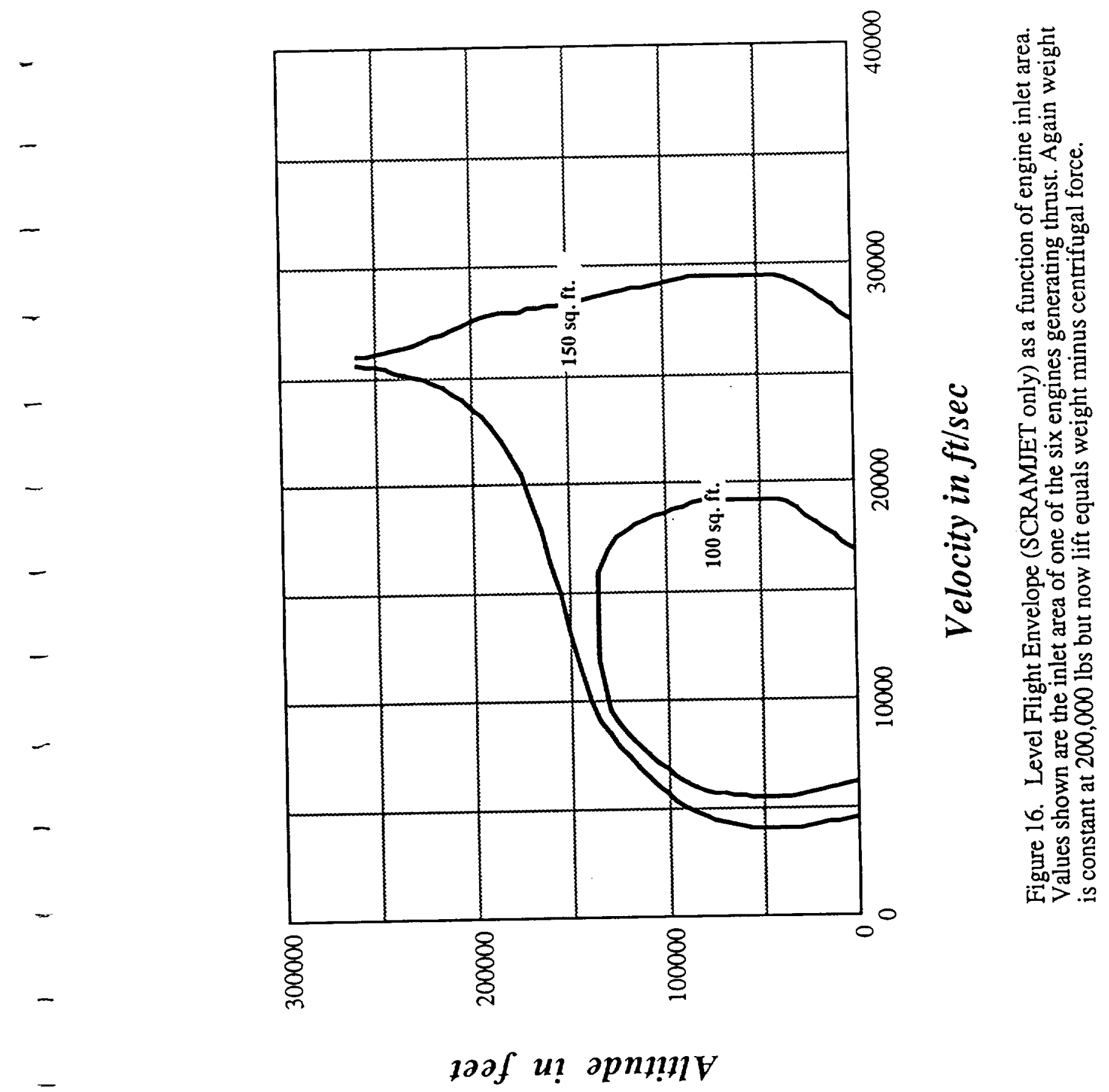




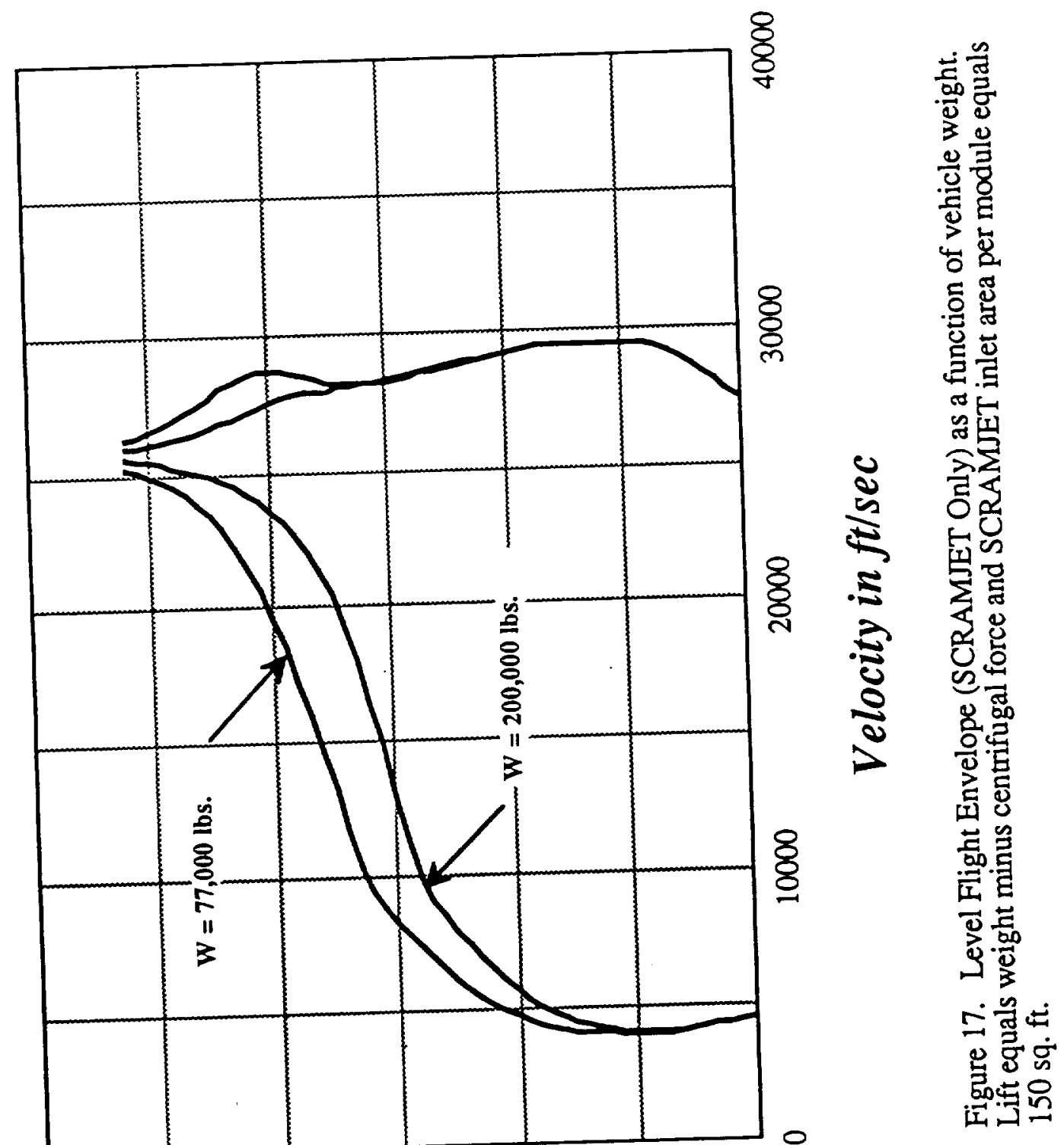

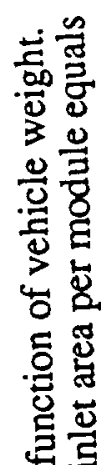

1ววf $u !$ opnI!1 $\mathrm{V}$ 


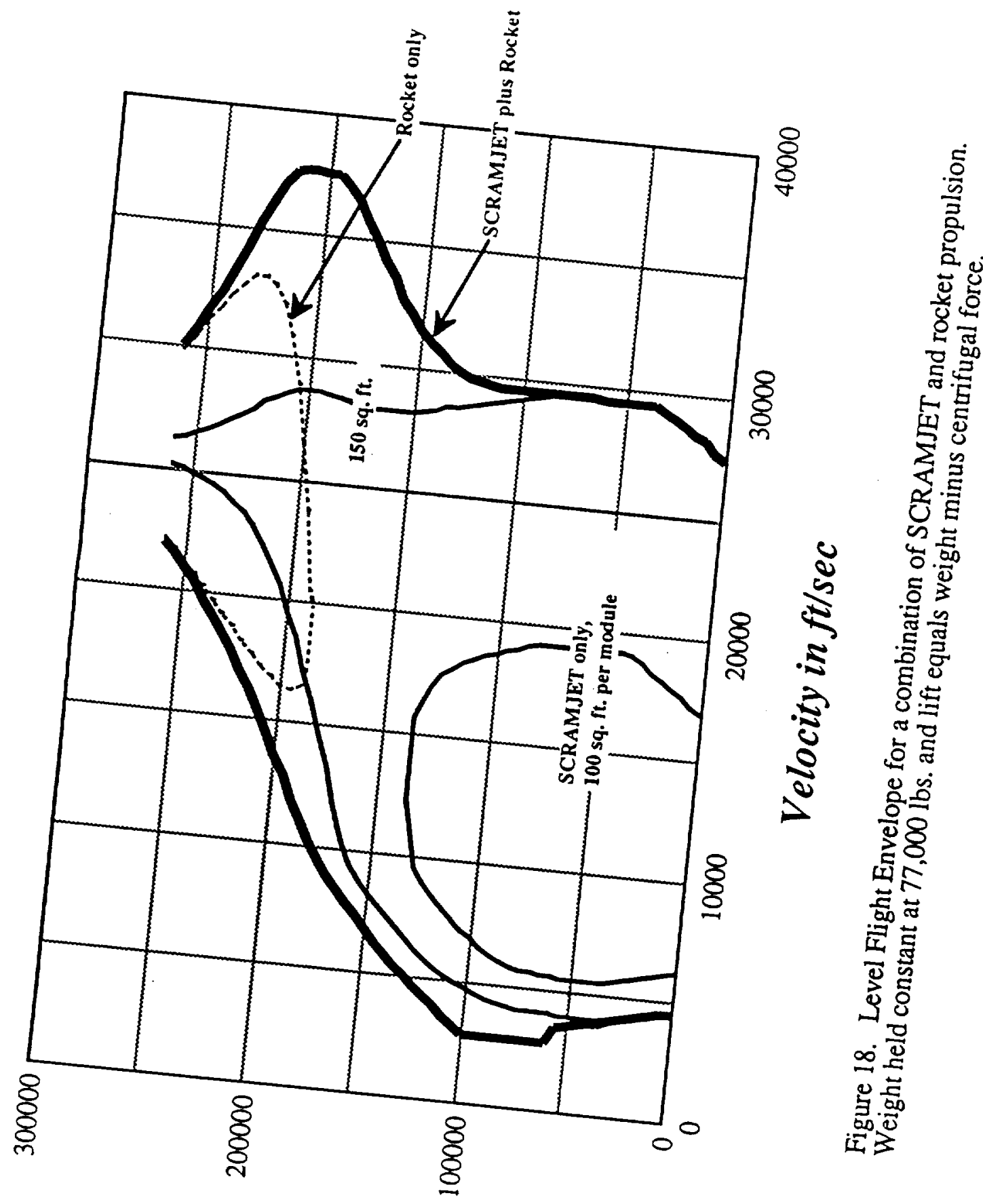

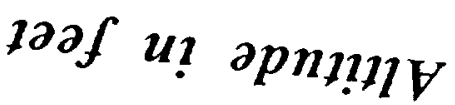




\section{Trajectory Optimization and Guidance Law Development}

For the remainder of this report we shall consider only one of the many tasks that will be required of the aerospace plane's guidance and control system, that of ascent to lowearth orbit. Such missions are routinely performed by rocket powered expendable, or in the case of the space shuttle, partly reusable vehicles. The NASP technology program emphasizes the goal of full reusability because of its pronounced impact on cost and operational flexibility. In fact, analyses of aerospace plane operations and support costs have shown that the greatest cost savings come from reduced turnaround time [8]. Advanced guidance and control systems are a key component to establishing rapid airlinelike launch operations. In order to reduce the cost of designing flight profiles, reduce the time required to respond to a changed payload or mission requirement and to improve the vehicle performance, on-board real-time optimal trajectory calculations will be required. The software must anticipate all possible mission requirements in order to avoid the need for mission dependent software modifications that lead to extensive preflight software testing requirements. Application of the necessary conditions for optimality in solving the trajectory optimization problem in general leads to a two-point boundary-value problem (TPBVP) that is difficult to solve. While some success in designing a reliable iterative algorithm to solve a TPBVP in an on-board computer has been achieved for orbit transfer [10], the diverse mission requirements of a general purpose aerospace plane will likely require that structured methods for order reduction be employed.

Energy state approximations and singular perturbation methods have proven to be useful in deriving on-board trajectory optimization algorithms. These methods also contribute considerable insight into the nature of the optimal profiles and their relation to vehicle aerodynamic and propulsion characteristics. Most of the studies performed thus far have been devoted to fighter aircraft performance optimization in the context of minimum time intercept $[14,40,41]$. These studies culminated in a series of piloted simulation evaluations at NASA Langley [42] and flight test demonstrations at NASA Dryden [43]. The 
techniques that were used are currently being applied to optimal orbit transfer maneuvers in the upper atmosphere [44].

Many of the modeling approximations employed for analysis of subsonic and supersonic aircraft optimal trajectories are not valid for a vehicle with hypersonic cruise and orbital capabilities. These can include the assumption of a flat Earth, constant vehicle weight, and a constant gravitational field. The problem of optimal airbreathing ascent to orbit is further complicated by the presence of severe trajectory constraints and the requirement for multiple modes of propulsion. SCRAMJET engines are incapable of operation below Mach 3 or 4. Alternate forms of propulsion are needed for take-off and landing, acceleration to supersonic speeds, and flight at the limits of the sensible atmosphere. The scope of this report is limited to flight above Mach 5; thus we shall not consider the required propulsion system transition in the supersonic regime. We shall, however, consider a transition in the hypersonic flight regime. As the vehicle accelerates toward orbital velocity it must continually gain altitude in order to avoid excessive aerodynamic heating. SCRAMJET performance degrades as altitude increases. The vehicle must convert to rocket propulsion as it leaves the atmosphere but the transition from SCRAMJET to rocket propulsion may well be advantageous prior to achieving orbital velocity. This transition to rocket propulsion during acceleration to orbital velocity must occur even if heating and other constraints are ignored since the fuel efficiency of a SCRAMJET degrades with increasing Mach number and will eventually fall below that of the rocket. The optimal point of propulsion system transition is identified in this report.

\subsection{Problem Formulation}

A good question to ask at this point is in what sense is the trajectory to be optimized? For the mission under consideration, namely ascent to orbit, one suitable goal is to minimize the total energy expended to achieve orbit. Since optimal space trajectories are well understood [45], we need only consider that portion of the flight within the sensible atmosphere, taken to be $\mathrm{h}<259,000$ feet. The minimum-fuel climb path is the selected goal for this analysis. 


\subsubsection{Dynamic Model}

Consider the equations of motion governing three-dimensional atmospheric flight of a point mass over a spherical rotating earth that are given below $[46,47]$. This set of equations is of an adequate complexity for our most detailed performance analysis. A stationary atmosphere and an inverse squared gravity field are assumed.

$\dot{\mathrm{r}}=\mathrm{V} \sin \gamma$

$\dot{\theta}=\frac{V \cos \gamma \cos \psi}{r \cos \phi}$

$\dot{\phi}=\frac{V \cos \gamma \sin \psi}{r}$

$\dot{V}=\frac{\left(\eta T \cos \varepsilon_{t}-D\right)}{m}-\frac{\mu \sin \gamma}{r^{2}}+\omega{ }^{2} r \cos \phi(\sin \gamma \cos \phi-\cos \gamma \sin \psi \sin \phi)$

$\dot{\gamma}=\frac{\left(\eta \mathrm{T} \sin \varepsilon_{\mathrm{t}}+\mathrm{L}\right) \cos \sigma}{\mathrm{mV}}-\frac{\mu \cos \gamma}{\mathrm{Vr}^{2}}+\frac{\mathrm{V} \cos \gamma}{\mathrm{r}}+2 \omega \cos \psi \cos \phi+$

$$
\frac{\omega^{2} \mathrm{r} \cos \phi}{\mathrm{V}}(\cos \gamma \cos \phi+\sin \gamma \sin \psi \sin \phi)
$$

$\dot{\psi}=\frac{\left(\eta T \sin \varepsilon_{1}+L\right) \sin \sigma}{m V \cos \gamma}-\frac{V \cos \gamma \cos \psi \tan \phi}{\mathrm{r}}+2 \omega(\tan \gamma \sin \psi \cos \phi-\sin \phi)-$ $\omega^{2} \mathrm{r} \cos \psi \sin \phi \cos \phi$

$$
\mathrm{V} \cos \gamma
$$


$\dot{\mathrm{m}}=-\mathrm{f}(\mathrm{r}, \mathrm{V}, \eta)$

The dots on the left-hand sides of equations (3-9) denote differentiation with respect to time. The state variables are: radius from the center of the Earth, $r$, longitude and latitude, $\theta$ and $\phi$ respectively, the flight velocity, $V$, flight path angle and heading angle, $\gamma$ and $\psi$ respectively, and vehicle mass, $m$. The control variables are engine throttle, $\eta$, vehicle lift, $\mathrm{L}$, and bank angle, $\sigma$. Figures 19 and 20 define the coordinate system and illustrate the geometric relationships between these variables.

Lift and drag are defined as:

$$
\begin{aligned}
& \mathrm{L}=\text { Lift }=1 / 2 \rho V^{2} C_{l} s \quad \text { where } C_{l}=C_{l}\left(\alpha, M_{\infty}\right) \\
& D=\text { Drag }=1 / 2 \rho V^{2} C_{d} s \quad \text { where } C_{d}=C_{d}\left(\alpha, M_{\infty}\right)
\end{aligned}
$$

The lift and drag coefficients, $C_{l}$ and $C_{d}$, are assumed to exhibit known functional relations to the vehicle angle of attack, $\alpha$, and the free stream Mach number, $M_{\infty}$. Furthermore, it is assumed that drag can be expressed in a conventional parabolic form as follows,

$$
\mathrm{D}=\mathrm{qsC}_{\mathrm{do}}+\mathrm{KL}^{2} / \mathrm{qs} \quad \mathrm{q}=\mathrm{pV}^{2} / 2
$$

where the symbol $q$ represents dynamic pressure, $s$ an aerodynamic reference area, $C_{d o}$ the zero-lift drag coefficient, and $\mathrm{K}$ the coefficient of the induced drag component. Altitude $(\mathrm{h})$ above mean sea level $\left(r_{0}\right)$ is given by,

$$
h=r-r_{0}
$$




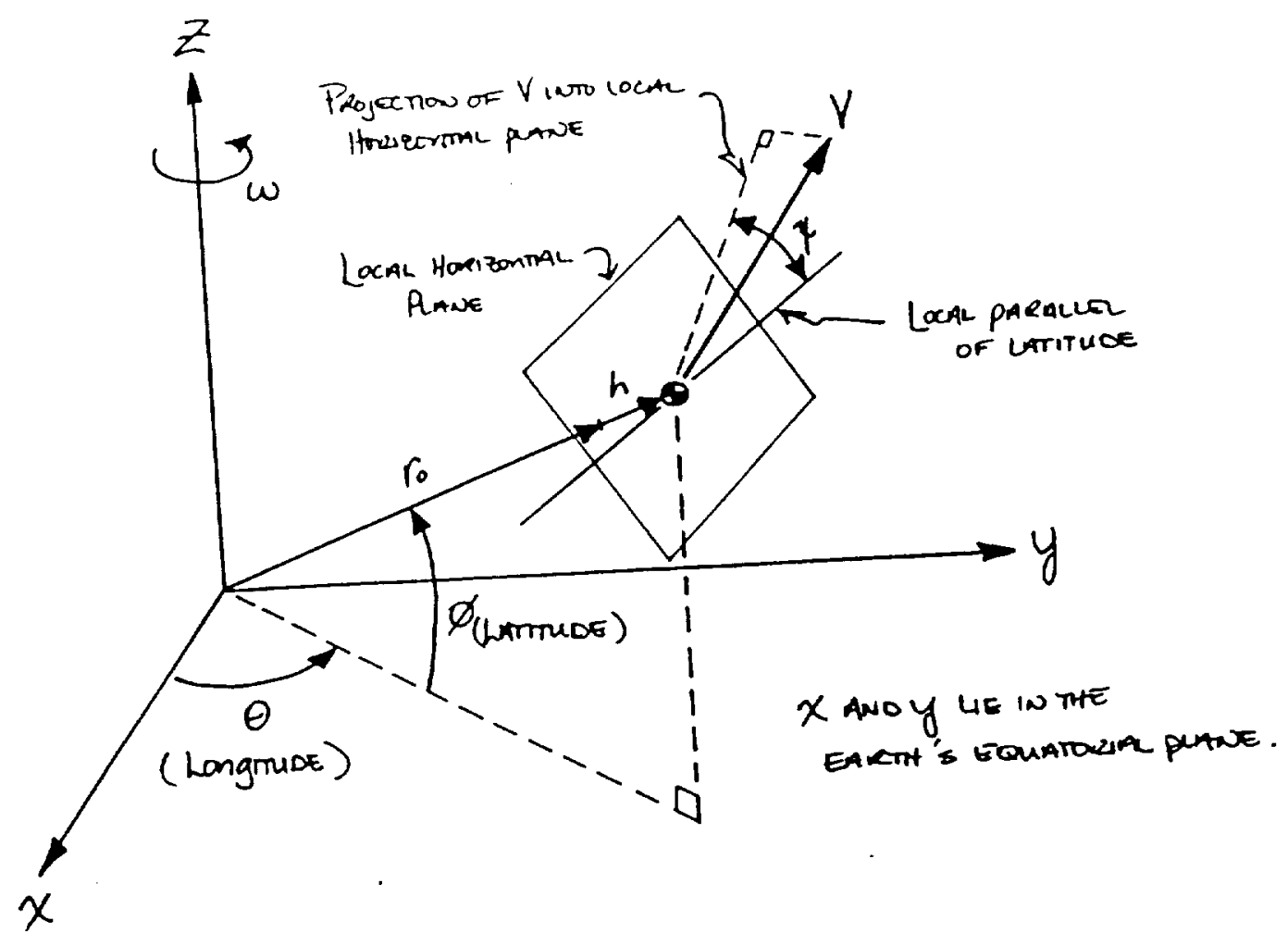

Figure 19. Earth Centered Coordinate System

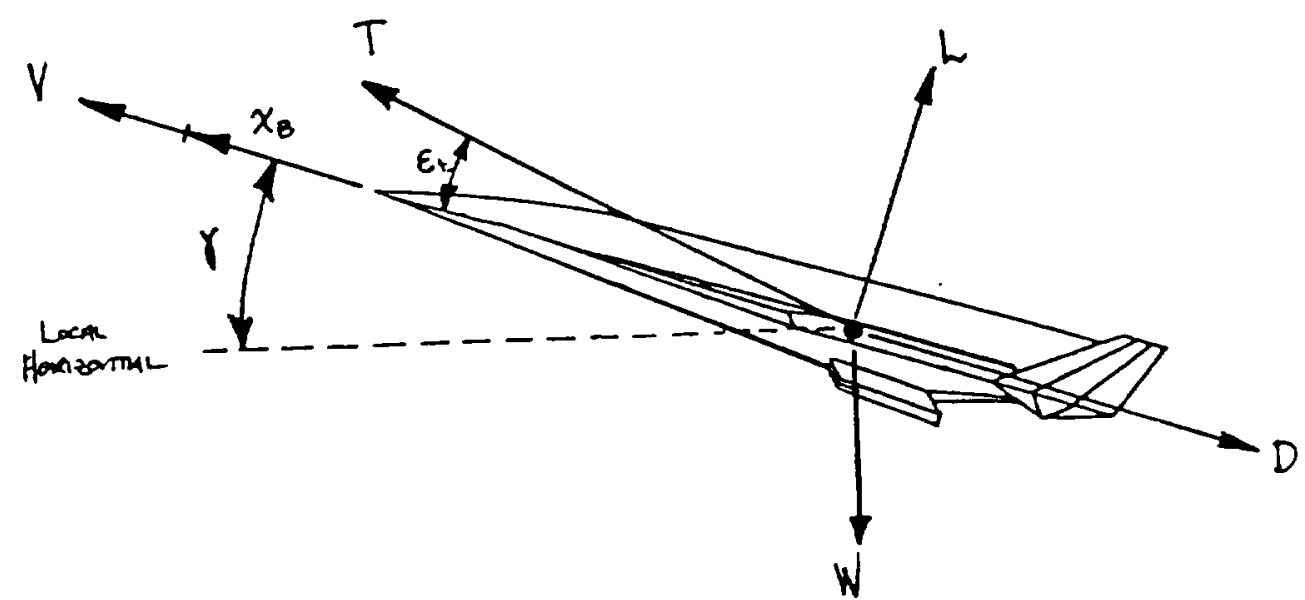

Figure 20. Aerodynamic and Propulsive Force Diagram 
Atmospheric density is represented by the symbol $\rho$ and varies in a known fashion with altitude. The constant $\mu$ represents the product of the Earth's mass and the universal gravitational constant. The reference point for zero gravitational potential is taken at infinity. The maximum thrust available, $T$, depends on the types of propulsion units being employed and on those parameters which influence the generation of thrust for each engine type. These may include Mach number, altitude, angle of attack, sideslip angle, $\beta$, and Q1a measure of fuel preheat occurring when the fuel is circulated as a coolant prior to combustion. In this set of equations it is assumed that the resulting thrust vector need not be aligned with the velocity vector, thus making an angle $\varepsilon$ with the longitudinal body axis as shown in Fig. 11. This vector is constrained, however, to lie in the vehicle's plane of symmetry. The variable $c$ shall represent specific fuel consumption (lbm/sec/lbf) which varies with the type of propulsion system, the Mach number, atmospheric density, and the throttle setting, $\eta$. Throttle setting shall be constrained in accordance with the type of propulsion system being employed. The options range from continuous variation over a specified range such as $0<\eta<1$ (typical for a turbojet), discrete variation, as in turning on or off one or more individual ramjet or SCRAMJET modules, or a fixed setting, as may occur with a rocket.

\subsubsection{Constraints}

Various constraints must be imposed on this dynamic model in order to simulate realistic flight. These constraints are required in order to maintain the vehicle's structural integrity, to prevent excessive heat loads or skin temperatures, to prevent aerodynamic stall or loss of control, to provide a suitable environment for human passengers, and to remain within particular engine operating conditions. A maximum dynamic pressure limit is considered in this report. Experience has shown, however, that if a dynamic pressure constraint is imposed then the freedom of control for optimizing performance is largely eliminated [48]. This is clearly indicated in Figure 9 of Section 4, page 23, where approximately $75 \%$ of the available flight envelope is eliminated by a maximum dynamic pressure constraint of 2000 psf. It is of interest to relax this constraint initially in order to gain insight into the nature of the optimal profiles, but a high dynamic pressure environment results in severe structural loads and unacceptable aerodynamic heating. Thus, it must be imposed as the analysis 
proceeds. Other constraints which will need to be incorporated into the analysis at a later date include acceleration limits, lift limits, and aerodynamic heating limits. A suitable model for aerodynamic heating has been identified in reference [49].

\subsubsection{Simplifying Assumptions}

With suitable models defined for the prediction of aerodynamic and propulsive forces and the vehicle's dynamic response, it is possible to simulate, using the above equations, the vehicle's flight given a control history, or to seek a control history which will optimize a performance index of interest. Rather than seeking solutions to trajectory optimization problems using the complex dynamic model described above, we wish to identify a model of reduced order that will yield an acceptable approximate solution. This is due to the fact that the resulting nonlinear optimal control problem proves to be very difficult to solve. Both direct and indirect methods of solution are available, but each proves to be computationally intensive. Furthermore, none of these solution methods leads to a guidance law in feedback form. For these reasons such methods are not suitable for on-board realtime implementation and we must turn to an approximate solution method. The energy state approximation has proven to be most useful in this regard [11], however, many of the other simplifying assumptions normally employed for model order reduction when considering transport or fighter aircraft may not be valid for a vehicle with hypersonic capabilities. These assumptions typically include [46]:

(1) Non-rotating Earth.

(2) Flat Earth.

(3) Constant acceleration due to gravity.

(4) Constant mass.

(5) Flight constrained to a vertical plane.

(6) Flight path angle small.

(7) Angle of attack small. 
(8) Thrust aligned with the velocity vector.

(9) Introduction of specific energy as a state variable

By adopting equations (3-9) it has already been assumed, without justification, that we have:

(10) Spherical Earth.

(11) Stationary atmosphere.

(12) Gravity field proportional to the inverse of the radius squared.

(13) The vehicle may be modeled as a point mass.

(14) Thrust vector constrained to lie in the plane of symmetry.

(15) The location of the vehicle center of mass is fixed (affects trim and thus the calculation of drag and elevon settings).

Let us examine each of the assumptions (1-9) individually, determine which may be employed for a hypersonic vehicle, and, where possible, determine the magnitude of the enror introduced by doing so. Consider first those assumptions which lend themselves to analytical investigation, such as (1-3).

Non-rotating Earth. Consider those terms in the given equations of motion that involve the rotational velocity of the earth, $\omega$. This rotation gives rise to two forces, or accelerations. The first, known as Coriolis acceleration, gives rise to terms involving $2 \omega \mathrm{V}$, which have an important impact on high speed, long range flight. The second, termed transport acceleration, gives rise to terms in $\omega^{2}$ r. Since for the earth, $\omega$ is small (approximately $7.27 \times 10^{-5} \mathrm{rad} / \mathrm{sec}$ ), the latter terms are most often neglected. The former is often retained for accuracy when computing the trajectory of a vehicle with near orbital capability. What is the maximum error that can be introduced into our analysis by neglecting either of these terms? For a given radial distance $r$, the transport acceleration depends on the latitude of the vehicle. The acceleration is zero at the poles and a maximum of value $\omega^{2} r$ when the vehicle is in the equatorial plane. The magnitude of this quantity is of 
the order $10^{-3} \mathrm{~g}_{0}$, where $\mathrm{g}_{\mathrm{o}}$ is the acceleration due to gravity at the Earth's surface. The Coriolis acceleration depends on the magnitude and the direction of the vehicle's velocity with respect to the Earth. It is zero when the flight path is parallel to the polar axis and a maximum when $\mathrm{V}$ is perpendicular to this axis. The magnitude of this value is given by $2 \omega \mathrm{V}$ and is of the order $10^{-1} \mathrm{~g}_{\mathrm{o}}$ at orbital velocities. Since we are primarily concerned with flight in the sensible atmosphere for which velocities must remain sub-orbital due to aerodynamic heating constraints, we shall set $\omega$ equal to zero in the above equations. This is the assumption of a non-rotating Earth [46].

Flat Earth. Further simplification results if the non-rotating Earth model discussed above is assumed flat. Ignoring the term $\mathrm{V}^{2} / \mathrm{gr}$ in the equations of motion amounts to ignoring the centrifugal force that contributes to the lift vector. As orbital velocity is approached the lift required is reduced to zero. Consequently the induced drag is also reduced to zero. This is an important effect which must be accounted for. Thus, we shall not make the assumption of a flat Earth.

Constant Acceleration due to Gravity. For flight within the atmosphere, the altitude of the vehicle remains small in comparison to the radius of the Earth. We may express the variation of acceleration due to gravity with altitude as $g=g_{0} r_{o}^{2} / r^{2}$ where $r=$ $r_{0}+h$. If we neglect $h$ as small compared to $r_{0}$, then $g$ remains constant with increasing altitude. For an altitude of 300,000 feet, $h / r_{0}=1.435 \times 10^{-2}$ and thus we induce an error less than that of neglecting the Coriolis acceleration. However, since including the variation of $g$ with altitude is a trivial matter, the gravity field shall be taken as proportional to the inverse of the radial distance, $r$, squared.

Constant Mass. This approximation is clearly invalid for a vehicle designed to achieve orbit. It is easily estimated assuming SCRAMJET propulsion and using an average specific impulse of 1500 seconds that half or more of the gross take-off weight for a vehicle with even a modest payload capability must be fuel weight [50].

Flight Constrained to a Vertical Plane. We shall find this approximation (bank angle of zero) to be most useful in getting started. However, it is expected that SCRAMJET 
performance will require that the trajectory follow the line of highest practical dynamic pressure, about $2000 \mathrm{psf}$. This is to ensure a high mass flow through the engine inlets. As a result the vehicle will be operating near minimum drag and with very low lift coefficients. As orbital velocity is approached, less and less lift will be required to support the vehicle. This may produce the need to fly at negative lift coefficients or to perform roll maneuvers to remain in the desired Mach number-altitude corridor [39]. It shall also be of interest to consider abort and plane change maneuvers later in this analysis. In either case we will require a three dimensional dynamic model. Throughout the remainder of this report, however, it shall be assumed that flight is constrained to a vertical plane. This assumption, when applied to the set of equations resulting from the assumption of a non-rotating Earth, provides for a decoupling of the altitude, velocity, mass, and flight path angle dynamics from those of longitude, latitude, and heading.

Angle of Attack and/or Flight Path Angle Small. It is expected, for the reasons cited above in the discussion of flight constrained to a vertical plane, that the vehicle angle of attack will remain small along the optimal trajectory. In fact, SCRAMJET performance may prove to be very sensitive to angle of attack at some Mach numbers and thus require strict constraints on vehicle angle of attack. The validity of these assumptions will have to be evaluated numerically.

Thrust Aligned with the Velocity Vector. This will be assumed until such time that a model for the normal component of SCRAMJET thrust is available. At very high Mach numbers, a large percentage of the vehicle afterbody will be used to expand the exhaust flow, reduce pressure drag, and increase the gross thrust. Since the pressures in these areas are above the free stream pressure, they could add lift and increase the lift-drag ratio and, consequently, cruise performance. In addition to making a large contribution to vehicle performance, propulsive lift may produce a nose-down pitching moment that must be trimmed out causing an aerodynamic penalty of increased trim drag. These effects may prove to be very important and should be considered in later analysis of this problem [39].

Introduction of Specific Energy as a State Variable. This approximation will be employed from the start and offers order reduction through the definition of a new state variable $[11,51]$. Its validity is tested in comparing the resulting approximate solution 
to exact numerical results. Experience in actual flights and comparison between various solutions for fighter aircraft have shown that the improvement in performance is minimal when the exact optimal solution is compared with suboptimal solutions obtained using this approximation.

\section{Simplified Dynamic Model}

Implementing the previously cited assumptions numbered $1,5,8$, and 9 in equations (39) results in a four state model in: radial distance from the center of the earth ( $r$ ), mass specific energy (E), flight path angle $(\gamma)$ and vehicle mass $(m)$. This set of equations is valid for atmospheric flight in a vertical plane of a point mass over a spherical non-rotating earth. The equations are:

$$
\begin{aligned}
& \dot{\mathrm{r}}=\mathrm{V} \sin \gamma \\
& \dot{\mathrm{E}}=\frac{\mathrm{V}(\mathrm{T}-\mathrm{D})}{\mathrm{m}} \\
& \dot{\gamma}=\frac{\mathrm{L}}{\mathrm{mV}}-\frac{\mu \cos \gamma}{\mathrm{Vr}^{2}}+\frac{\mathrm{V} \cos \gamma}{\mathrm{r}} \\
& \dot{\mathrm{m}}=-\mathrm{f}(\mathrm{r}, \mathrm{V}, \eta)
\end{aligned}
$$

It is assumed that the atmosphere is stationary and that the thrust vector is directed along the path (i.e. $\varepsilon_{\mathrm{t}}=0$ ). In equation (15), mass specific energy, $E$, is employed as a state variable in place of velocity, $V$, where 


$$
E=\frac{V^{2}}{2}-\frac{\mu}{r}
$$

hence $V$ is to be taken as

$$
V=[2(E+\mu / r)]^{1 / 2}
$$

wherever it appears in this analysis unless otherwise noted. A dual-mode propulsion system is assumed. This system consists of a bank of SCRAMJET engine modules assumed to operate continuously at stoichiometric conditions above Mach 10 and a rocket engine that can be turned on or off as desired. The total fuel flow rate ( $f$ ) and $T$ are represented by

$$
\begin{aligned}
& T=T_{s}+\eta T_{r} \quad ; \quad \eta \in[0,1] \\
& f=c_{s} T_{s}+\eta c_{r} T_{r}
\end{aligned}
$$

where thrust specific fuel consumption is represented by $c_{s}$ for the SCRAMJET and $c_{r}$ for the rocket. The control variables are now rocket engine throttle $(\eta)$ and vehicle lift $(L)$. The objective shall be to minimize the fuel consumed in an unconstrained energy climb. The performance index is given by,

$$
J=-m\left(t_{f}\right) \quad ; \quad t_{f} \text { free }
$$

\subsection{Application of Singular Perturbation Theory}

The task is to determine the controls, $\eta$ and $L$, so that equation (22) is minimized. Even though the order of the dynamic model has been reduced from seven to four, a 
TPBVP, that proves difficult to solve, again results from applying the necessary conditions for optimality. As stated earlier, this approach is computationally intensive and not well suited to on-board real-time implementation. For this reason we seek further model order reduction through the application of singular perturbation theory. This technique is detailed in references [12-14]. Regarding energy and mass as slow variables and altitude and flight path angle as fast, we introduce the perturbation parameter $\varepsilon$, nominally one, in equations $(14-17)$.

$$
\begin{aligned}
& \dot{\mathrm{E}}=\frac{V(T-D)}{\mathrm{m}} \\
& \dot{\mathrm{m}}=-\mathrm{f}(\mathrm{r}, \mathrm{V}, \eta) \\
& \dot{\varepsilon \gamma}=\frac{\mathrm{L}}{\mathrm{mV}}-\frac{\mu \cos \gamma}{\mathrm{Vr}^{2}}+\frac{\mathrm{V} \cos \gamma}{\mathrm{r}} \\
& \dot{\mathrm{E}}=\mathrm{V} \sin \gamma
\end{aligned}
$$

The first-order necessary conditions for optimality are:

$$
\begin{gathered}
\mathrm{H}=\lambda_{\mathrm{E}} \dot{\mathrm{E}}+\lambda_{\mathrm{m}} \dot{\mathrm{m}}+\lambda_{\mathrm{r}} \mathrm{V} \sin \gamma+\lambda_{\gamma} \dot{\gamma}=0 \\
\dot{\lambda}_{\mathrm{E}}=-\frac{\partial \mathrm{H}}{\partial \mathrm{E}} \quad \dot{\lambda}_{\mathrm{m}}=-\frac{\partial \mathrm{H}}{\partial \mathrm{m}} \\
\lambda_{\mathrm{m}}\left(\mathrm{t}_{\mathrm{f}}\right)=-1.0 \\
\dot{\varepsilon \lambda_{\gamma}=-\frac{\partial \mathrm{H}}{\partial \gamma}} \quad \dot{\lambda}_{\mathrm{r}}=-\frac{\partial \mathrm{H}}{\partial \mathrm{r}}
\end{gathered}
$$




$$
\frac{\partial \mathrm{H}}{\partial \mathrm{L}}=0
$$

The analysis proceeds by examining the necessary conditions for optimality on two separate time scales, $t$ and $\tau=v \varepsilon$, for $\varepsilon=0$. The results are termed the reduced and boundary layer solutions, respectively.

\subsubsection{Reduced Solution}

\section{The Unconstrained Case}

Setting $\varepsilon=0$ in (23-26) reduces the order of the dynamic system to two. Altitude and flight path angle dynamics are assumed fast in comparison to energy and mass dynamics, and altitude now takes on the role of a control variable. The differential equations (25) and (26) are reduced to algebraic equations which yield the following relations:

$$
\begin{aligned}
& \gamma_{0}=0 \\
& L_{0}=m\left(\frac{\mu}{r^{2}}-\frac{V^{2}}{r}\right)
\end{aligned}
$$

The subscripted zeros denote reduced solution values in this context and are omitted where not deemed necessary for clarity. Thus, in the reduced solution, flight path angle is assumed to remain zero along the optimal path and lift is completely determined as weight minus centrifugal force. The reduced solution Hamiltonian is given by

$$
\mathrm{H}_{\mathrm{o}}=\lambda_{\mathrm{E}} \dot{\mathrm{E}}+\lambda_{\mathrm{m}} \dot{\mathrm{m}}=0
$$

where

$$
\lambda_{\mathrm{m}}\left(\mathrm{t}_{\mathrm{f}}\right)=-1.0
$$

We can use the fact that $H_{o}$ is a constant of motion to express one of the unknown costates in terms of the other as follows, 


$$
\lambda_{E}=\lambda_{m}\left[\frac{f m}{V(T-D)}\right]
$$

Note that when considering an energy climb (i.e. a positive energy rate) $\lambda_{E}$ must always have the same sign as $\lambda_{m}$. Furthermore since $\lambda_{m}$ represents the variation in the performance index, $\mathrm{J}$, with respect to mass, it cannot change sign (i.e. it is not possible for a reduction in vehicle mass due to fuel expenditure along the climb path to increase the final mass of the vehicle). Given that $J=-m\left(t_{f}\right), \lambda_{m}$, and thus $\lambda_{E}$, must remain negative. Using this sign information it can be shown (see Appendix $B$ ) that maximization of the function $\mathrm{dE} / \mathrm{dt}$ divided by $\mathrm{dm} / \mathrm{dt}$ with respect to the controls is equivalent to minimizing the reduced solution Hamiltonian, (34), with respect to the controls. Thus, satisfaction of the minimum principle is reduced to the following operation,

$$
h_{0}, \eta_{0}=\left.\arg \max _{h, \eta} \frac{V(T-D)}{f}\right|_{\substack{E=\text { constant } \\ T>D}}
$$

In addition to being relieved of having to solve a TPBVP, the two-dimensional search for values of rocket throttle setting and altitude that maximize the function identified in equation (37) while holding energy constant and constraining thrust to remain greater than drag can be performed off-line and stored as a function of energy level and mass. The use of equation (33) in such an off-line calculation requires that an estimate of vehicle mass be generated as a function of energy level. A means for doing so is reported on page 44 of this report. It turns out that with the current vehicle model being employed, the reduced solution is relatively insensitive to mass variation. This is due to the fact that the vehicle tends to operate at very low lift coefficients. As a result, when a change in mass effects the required lift through equation (33), the change in drag, which effects the maximization in (37), is only slight since the lift curve slope with respect to drag is very shallow at low values of lift coefficient (see Figure A3).

Note that rocket throttle setting, $\eta$, appears linearly in the Hamiltonian. This indicates a bang-bang control solution for rocket throttle setting and the possibility that singular arcs 
exist. It is shown in the next sub-section of this report that intermediate values of rocket throttle setting, as would occur along an optimizing singular arc, are in fact non-optimal. Furhermore, in the sub-section that follows, an analytic rocket throttle switching function is derived that eliminates the need to perform the maximization indicated in equation (37) with respect to throttle setting. Thus, equation (37) reduces to the form:

$$
h_{o}=\left.\arg \max _{h} \frac{V(T-D)}{f}\right|_{\substack{T>D \\ \eta=\eta^{*}}}
$$

\section{Examination of Possible Singular Arcs}

Because throttle setting appears linearly in the Hamiltonian, the necessary condition that the partial derivative of $\mathrm{H}_{\mathrm{o}}$ with respect to throttle setting be zero yields no control solution directly. Instead we must examine the sign of this partial derivative in order to determine the optimal throttle setting. The optimal control necessarily occurs at one control boundary or the other and may switch from one to the other any number of times. There is also the possibility that singular arcs exists. That is, we may find that the afore mentioned partial derivative is zero over a finite duration of time, hence no sign can be determined. In such a case intermediate values of throttle setting may occur and it must be determined whether such values are optimal. The switching condition $(S)$ is determined as follows [52]:

$$
\frac{\partial H_{0}}{\partial \eta}=\frac{\partial}{\partial \eta}\left[\lambda_{E} \frac{V(T-D)}{m}+\lambda_{m}(-f)\right]=T_{r}\left[\lambda_{E} \frac{V}{m}-\lambda_{m} c_{r}\right] \text {, where } T_{r} \geq 0
$$

The sign of the partial derivative of $\mathrm{H}_{0}$ with respect to $\eta$ is determined by the sign of the last bracketed term in equation (39), which is termed the switching function, $\mathrm{S}$.

$$
\frac{\partial H_{o}}{\partial \eta} \Rightarrow S=\lambda_{E}\left(\frac{V}{m}\right)-\lambda_{m} c_{r}
$$


The rocket control solution can be summarized as follows:

$$
\begin{array}{rc}
\eta=1 & \text { when } \quad S<0 \\
0 \geq \eta \leq 1 & \text { if } S \equiv 0 \text { for a finite duration of time } \\
\eta & =0 \quad \text { when } \quad S>0
\end{array}
$$

Next we would like to show that singular arcs, although they do exist in this problem, are not optimizing. By definition, the case in which an extremal arc $\left(\mathrm{H}_{\mathbf{u}}=0\right)$ occurs along which the matrix $\mathrm{H}_{\mathrm{uu}}$ is singular is termed a singular arc. Such arcs satisfy the necessary condition on convexity, but not the strengthened condition; that is, $\mathrm{H}_{\mathrm{uu}}$ is only positive semi-definite. Additional tests are usually required to determine if a singular arc is optimizing or not $[52,53]$. In this case it can simply be shown that $\mathrm{H}_{\mathrm{uu}}$ is not positive semidefinite. Thus, singular arcs are non-optimizing. We proceed as follows.

Consider again the reduced solution Hamiltonian:

$$
\mathrm{H}_{\mathrm{o}}=\lambda_{\mathrm{E}} \dot{\mathrm{E}}+\lambda_{\mathrm{m}} \dot{\mathrm{m}}=0
$$

Let us examine $H_{u u}$ where $u^{T}=[V, \eta]$ ]. Note that in equations (14-17), velocity, $V$, was eliminated through equation (19) when implementing the energy state approximation. An equivalent formulation of the reduced solution reported above is obtained by eliminating radial distance, $r$, and retaining velocity, $V$. In that case, $V$ rather than $h$, acts as a controllike variable in the reduced solution. It is much more convenient to use the latter formulation for this argument. In this context, the subscript notation, $\mathrm{H}_{\mathrm{uu}}$, denotes partial differentiation as follows:

$$
H_{x y}=\frac{\partial}{\partial x}\left(\frac{\partial H}{\partial y}\right)^{T}
$$

Expanding $\mathrm{H}_{\mathrm{uu}}$ into its matrix representation yields,

$$
H_{u}=\left[\begin{array}{ll}
H_{v v} & H_{\eta v} \\
H_{V \eta} & H_{\eta \eta}
\end{array}\right]
$$


Recall that it is necessary for optimality that $\mathrm{H}_{\mathrm{uu}}$ be at least positive semidefinite. The determinant of the symmetric portion of $\mathrm{H}_{\mathrm{uu}}$ must be greater than or equal to zero for positive semidefiniteness. In the case of a singular arc, $\mathrm{H}_{\eta \eta} \equiv 0$ by definition. Thus, the only terms that contribute to the determinant of the symmetric portion of $\mathrm{H}_{\mathrm{uu}}$ are the offdiagonal terms of the matrix shown in equation (44).

$$
\operatorname{det} \mathrm{H}_{\mathrm{u}_{\text {symmetric }}}=-\left[\left(\mathrm{H}_{\eta \mathrm{V}}\right)^{2}+2 \mathrm{H}_{\eta \mathrm{V}} \mathrm{H}_{\mathrm{V} \eta}+\left(\mathrm{H}_{\mathrm{V} V}\right)^{2}\right]
$$

Carrying out the indicated partial differentiation, and ignoring the weak dependence of $T_{r}$ and $C_{r}$ on $h$ yields:

$$
\begin{aligned}
& \frac{\partial H_{o}}{\partial \eta}=\frac{\lambda_{E} V T_{r}}{m}-\lambda_{m} c_{r} T_{T} \\
& \frac{\partial^{2} H_{o}}{\partial \eta \partial V}=\frac{\lambda_{E} T_{r}}{m}
\end{aligned}
$$

It is straightforward to show that

$$
\frac{\partial^{2} H_{o}}{\partial V \partial \eta}=\frac{\partial^{2} H_{o}}{\partial \eta \partial V}
$$

Equation (47) also holds if the dependence of $T_{r}$ and $C_{r}$ on $h$ is accounted for. Thus

$$
\operatorname{det} \mathrm{H}_{\mathbf{u}_{\text {symmetric }}}=-\left(4 \mathrm{H}_{\mathrm{V} \eta}\right)^{2}
$$

which is negative for non-zero values of $\mathrm{H}_{V_{\eta}}$. The expression for $\mathrm{H}_{V_{\eta}}$ is as follows, where the approximation results from neglecting the weak dependence of $T_{r}$ and $C_{r}$ on $h$ 


$$
H_{V \eta}=\lambda_{m}\left[\left(\frac{f}{V(T-D)}-c_{r V}\right) T_{r}+\left(\frac{f}{(T-D)}-c_{r}\right) T_{r V}\right] \cong \frac{\lambda_{E} T_{r}}{m}
$$

In general the bracketed term of (49) is non zero. It follows from the condition $\mathrm{H}_{\mathrm{o}}=0$ that if $\lambda_{E}=0$ then $\lambda_{m}=0$, and visa versa, which is a trivial case. Thus $H_{u u}$ is not positive semidefinite, and intermediate values of rocket throttle setting are not optimal $[54,55]$.

\section{Analytic Form for Throttle Switching Condition}

Consider again the switching condition given in equation (40),

$$
S=\lambda_{E}\left(\frac{V}{m}\right)-\lambda_{m} c_{r}
$$

Using equation (36) we may eliminate the costate $\lambda_{E}$ from equation (50) to yield,

$$
S=\lambda_{m}\left[\frac{f}{T-D}-c_{r}\right]
$$

As stated previously, $\lambda_{m}$ represents the variation in the performance index, $J$, with respect to mass, and $\lambda_{m}\left(t_{f}\right)=-1$. Clearly, since $J=-m\left(t_{f}\right), \lambda_{m}$ cannot change sign (i.e. it is not possible for a reduction in vehicle mass as fuel is expended along the climb path to increase the final mass of the vehicle). Therefore $\lambda_{m}$ must always be less than zero. The sign of $\mathrm{H}_{\eta}$ is thus determined by the bracketed term in equation (51). Substituting for thrust, $T$, and fuel flow rate, $\mathrm{f}$, using equations (20) and (21), and taking into account (41), yields the following analytic form for the rocket throttle switching condition, 


$$
\begin{aligned}
& \eta=0 \text { if }\left[\frac{c_{r}-c_{s}}{c_{r}}\right] T_{S}>D \\
& \eta=1 \text { if }\left[\frac{c_{r}-c_{s}}{c_{r}}\right] T_{S}<D
\end{aligned}
$$

\section{Estimation of States and Costates in the Reduced Solution}

In the above solution, the variables $L_{0}$ and $\gamma_{0}$ are first eliminated before the maximization in (38) is performed. An alternative viewpoint is to adjoin the constraints (that the right hand sides of (25) and (26) are zero) to the Hamiltonian. That is

$$
\mathrm{H}_{\mathrm{o}}=\lambda_{\mathrm{E}_{0}} \dot{\mathrm{E}}+\lambda_{\mathrm{m}_{0}} \dot{\mathrm{m}}+\lambda_{\mathrm{r}_{0}} \mathrm{~V} \sin \gamma+\lambda_{\gamma_{0}} \dot{\gamma}
$$

Associated with this formulation are the additional necessary conditions (see 30 and 31):

which result in

$$
\frac{\partial \mathrm{H}_{\mathrm{o}}}{\partial \gamma}=\frac{\partial \mathrm{H}_{\mathrm{o}}}{\partial \mathrm{L}}=0
$$

$$
\begin{aligned}
& \lambda_{\mathrm{r}_{0}}=0 \\
& \lambda_{\gamma_{0}}=\lambda_{\mathrm{E}_{0}}\left[\frac{2 \mathrm{KV} \mathrm{V}^{2}}{\mathrm{qs}}\right]
\end{aligned}
$$

Using (34) we may solve for $\lambda_{E_{0}}$ in terms of $\lambda_{m_{0}}$

$$
\lambda_{E_{0}}=\lambda_{m_{0}}\left[\frac{f m}{V(T-D)}\right]
$$

where $\lambda_{\text {mo }}$ satisfies (see equation 28 ) 


$$
\dot{\lambda}_{\mathrm{m}}=-\frac{\partial \mathrm{H}_{\mathrm{o}}}{\partial \mathrm{m}}=\lambda_{\mathrm{E}_{\mathrm{o}}}\left(\frac{\mathrm{V}}{\mathrm{m}^{2}}\right)\left[\mathrm{T}-\mathrm{D}+\frac{2 \mathrm{KL}_{\mathrm{o}}^{2}}{\mathrm{qs}}\right]
$$

An approximate integral of (58) can be obtained by noting that the term $\mathrm{KL}_{\mathrm{o}}{ }^{2} / \mathrm{qs}$ in (58) represents the induced drag component (see equation 12), which for small angles of attack is small in comparison to the zero lift drag component. Ignoring this term and using (57) to eliminate $\lambda_{E},(58)$ becomes

$$
\frac{\dot{\lambda}_{\mathrm{m}}}{\lambda_{\mathrm{m}}}=-\frac{\dot{\mathrm{m}}}{\mathrm{m}} \quad \text { where again as in }(35) \quad \lambda_{\mathrm{m}}\left(\mathrm{t}_{\mathrm{f}}\right)=-1.0
$$

Integrating both sides leads to the approximation

$$
\lambda_{\mathrm{m}_{0}} \cong-\frac{\mathrm{m}\left(\mathrm{t}_{\mathrm{f}}\right)}{\mathrm{m}}
$$

Note that expressions (56), (57), and (60) each depend on knowledge of $m\left(t_{f}\right)$. An estimate of $m\left(t_{f}\right)$ can be formed by integration of $\mathrm{dm} / \mathrm{dt}$ over $\mathrm{dE} / \mathrm{dt}$ along the reduced solution as follows:

$$
\Delta m=\int_{E_{0}}^{E_{f}}\left(\frac{d m}{d E}\right) d E=\int_{E_{0}}^{E_{f}}\left(\frac{\dot{m}}{\dot{E}}\right) d E
$$

then

$$
m\left(t_{f}\right)=m\left(t_{o}\right)-\Delta m
$$

In a similar fashion it is possible to estimate the time required to gain energy,

$$
\Delta T=\int_{E_{0}}^{\mathrm{F}_{1}} \frac{\mathrm{dt}}{\mathrm{dE}} \mathrm{dE}=\int_{\mathrm{E}_{0}}^{\mathrm{E}_{\mathrm{T}}} \frac{\mathrm{dE}}{\dot{\mathrm{E}}}
$$


It is also of interest to form an estimate of the flight path angle along the reduced solution. This may be accomplished by first solving for the flight path angle, $\gamma$, in equation (14).

$$
\dot{\mathrm{h}}=\mathrm{V} \sin \gamma \quad \Rightarrow \quad \gamma=\sin ^{-1}\left(\frac{\dot{\mathrm{h}}}{\mathrm{V}}\right)
$$

Using the chain rule for differentiation one can write,

$$
\dot{\mathrm{h}}=\frac{\mathrm{dh}}{\mathrm{dE}} \dot{\mathrm{E}}
$$

where $\mathrm{dh} / \mathrm{dE}$ can be approximated by considering two closely spaced energy levels

$$
\frac{\mathrm{dh}}{\mathrm{dE}} \equiv \frac{\mathrm{h}_{2}^{*}-\mathrm{h}_{1}^{*}}{\Delta \mathrm{E}}
$$

In equation (66) the superscripted asterisks denotes the reduced solution optimal altitude for a given energy level. Using equations (65) and (66) in (64), we find that the flight path angle at a given energy level, $E_{2}$, can be estimated as follows,

$$
\gamma_{2} \cong \sin ^{-1}\left[\frac{h_{2}^{*}-h_{1}^{*}}{\Delta E} \frac{\dot{E}_{2}}{V_{2}^{*}}\right]
$$

\section{Inclusion of a Maximum Dynamic Pressure Constraint}

Recall the definition given for dynamic pressure, $q$, and velocity, $V$ :

$$
q=1 / 2 \rho V^{2}=q(E, r) \quad \text { where } V=[2(E+\mu / r)]^{1 / 2}
$$

We wish to constrain $q$ so that $q \leq q_{\max }$. Thus we wish to enforce a constraint on a function of the state and control variables ( $E$ and $r$ [i.e., h], respectively) all along the reduced solution. That is 
Minimize J, subject to the constraint, $\quad C(E, h)=q-q_{\max } \leq 0$

In order to proceed, we adjoin this constraint to the reduced solution Hamiltonian with a Lagrange multiplier as follows [52],

$$
\mathrm{H}_{\mathrm{o}}=\lambda_{\mathrm{E}} \dot{\mathrm{E}}+\lambda_{\mathrm{m}} \dot{\mathrm{m}}+\lambda_{\mathrm{q}} \mathrm{C}=0 ; \lambda_{\mathrm{m}}\left(\mathrm{t}_{\mathrm{f}}\right)=-1.0
$$

where

$$
\begin{array}{ll}
\lambda_{\mathrm{q}}=0, & \mathrm{C}<0 \\
\lambda_{\mathrm{q}} \geq 0, & \mathrm{C}=0
\end{array}
$$

This constraint does not affect the throttle switching condition in (52), but it does limit the search space over which the maximization of equation (38) takes place:

$$
h_{0}=\left.\arg \max _{h} \frac{V(T-D)}{f}\right|_{\substack{E \\ T>D \\ \eta=\eta^{*} \\ q \leq q \max }}
$$

The constraint multiplier $\lambda_{q}$ can be evaluated from the following condition,

$$
\frac{\partial H_{o}}{\partial h}=0 \Rightarrow \lambda_{q}=-\frac{\frac{\partial H_{o}}{\partial h}}{\frac{\partial q}{\partial h}}
$$

\section{Reduced Solution: Numerical Results}

Reduced solution trajectories for the vehicle modeled in Sections 2-4 were generated by carrying out the maximization process indicated in equations (38) and (72) over the energy range corresponding to $\mathrm{V}=5000 \mathrm{ft} / \mathrm{sec}$ at $\mathrm{h}=0$ to $\mathrm{V}=25,000 \mathrm{ft} / \mathrm{sec}$ at $\mathrm{h}=200,000 \mathrm{ft}$. The unconstrained case and the case for which dynamic pressure is constrained to be less than 2000 p sf are presented in Figure 21. 
The unconstrained case begins at an altitude of 36,000 feet and initially exhibits an acceleration at constant altitude. This is followed by a rapid transition in altitude at approximately Mach 10 due in part to the modeling of atmospheric properties (an isothermal layer is assumed from $h=36,000 \mathrm{ft}$ to $82,000 \mathrm{ft}$ ) and in part to a transition in SCRAMJET performance. Below Mach 10 optimum engine performance is achieved at a fuel-to-air ratio less than stoichiometric whereas above Mach 10 the calculated fuel-to-air ratio exceeds its stoichiometric value. It is assumed that no additional thrust is available at a higher ratio, thus the fuel-to-air ratio is constrained in the engine model to remain less than or equal to its stoichiometric value. This is indicated graphically in Figure 10 on page 22 . From this point in the trajectory a slow steady climb ensues until the altitude exceeds $82,000 \mathrm{ft}$, at which point a constant altitude acceleration is again optimal (since the assumed isothermal layer terminates). Note that at a velocity of approximately $21,000 \mathrm{ft} / \mathrm{sec}$ the rocket engine is deemed advantageous and is tumed on.

At a velocity of $22,000 \mathrm{ft} / \mathrm{sec}$. Operational efficiency dictates a switch in operating altitude. This is indicated in Figure 21 by a vertical line, and represents a constant energy instantaneous altitude transition. This altitude discontinuity also occurs in the q constrained case and in the case of SCRAMJET propulsion alone (no rocket) as illustrated in Figure 22. The mechanism causing this discontinuity is depicted in Figure 23 where the function to be maximized with respect to altitude[see equations (38) and (72)] is plotted as a function of altitude for three particular energy levels. Note that these curves terminate on the left at approximately 135,000 feet - this corresponds to the dynamic pressure constraint boundary. As the energy level increases, the local maximum at approximately 200,000 feet overcomes the functional maximum previously occurring at the constraint boundary. The shape of the curves presented in Figure 23 results from the interplay of the modeled thrust, drag, and fuel flow dependencies on altitude and velocity. The addition of rocket thrust shifts this altitude discontinuity to a lower energy level. The basic result is that at a sufficiently high Mach number, degrading engine performance and increasing drag causes a switch in the optimum operating conditions from high thrust/high drag to low thrust/low drag. Given an initial weight of $200,000 \mathrm{lbs}$, the weight of fuel expended along the unconstrained path shown in Figure 21 is estimated as $115,875 \mathrm{lbs}$. (58\% of the take-off gross weight). The time of flight was estimated as 659 seconds. The most efficient cruise point was identified at approximately Mach 10 at 36,000 feet. The percent of take-off gross 
weight expended in achieving the same energy for the SCRAMJET only case was estimated as $67 \%$. The time required in this case was estimated as 2388 seconds.

Optimal SCRAMJET performance demands flight at an altitude for which the highest mass flow of air is available. Consideration of the airframe, however, dictates that dynamic pressure be limited, the highest practical value being approximately 2000 psf. Maximization of (72) subject to $q \leq 2000 \mathrm{psf}$ results in the constrained trajectory presented in Figure 21 . Here the vehicle follows the $q$ constraint all the way up to $\mathrm{V}=21,500 \mathrm{ft} / \mathrm{sec}$, (the rocket being advantageous above a velocity of $19,500 \mathrm{ft} / \mathrm{sec}$ ) at which point climb out of the atmosphere is initiated for the same reason as cited for the unconstrained case. Note that temperature limits on the nose and wing leading edges (not enforced) may force climb out of the atmosphere at a lower Mach number. Total fuel expended along the constrained path for the same initial weight is estimated as $120,536 \mathrm{lbs}$. ( $60 \%$ of the take-off gross weight). This corresponds to 2 percent more of the total gross weight being expended in achieving the specified final energy over the unconstrained case. It should be noted that the time required to achieve the final energy, estimated as 1372 seconds, is much greater along the constrained path. The difference in time between the constrained and unconstrained case is 714 seconds. This difference results because less excess thrust is available to accelerate the vehicle in the constrained case. The most efficient cruise point was again identified at approximately Mach 10 but now on the q constraint boundary at about 95,000 feet.

Figures 24 and 25 present the required lift coefficient and the corresponding angle of attack required to maintain lift equals weight minus centrifugal force for the q constrained case as a function of energy level. As expected, the vehicle operates at very low values of lift coefficient. The corresponding angle of attack remains less than four degrees over the entire trajectory. Figures 26 and 27 depict the thrust to drag ratios computed along the unconstrained and $q$ constrained cases. Of note is the fact that the average thrust to drag ratio of the entire energy change is 2.28 in both cases. The average thrust to drag ratio for the SCRAMJET only case is 1.62 . The estimated flight path angle history required to follow the $q$ constrained reduced solution climb path is presented in Figure 28. The spike at energy level 36 is generated by the altitude discontinuity discussed earlier, and even in this region, the flight path angle is estimated to remain less than three degrees. Clearly the assumption of a small flight path angle and small angle of attack is valid for the reduced solution climb path. 


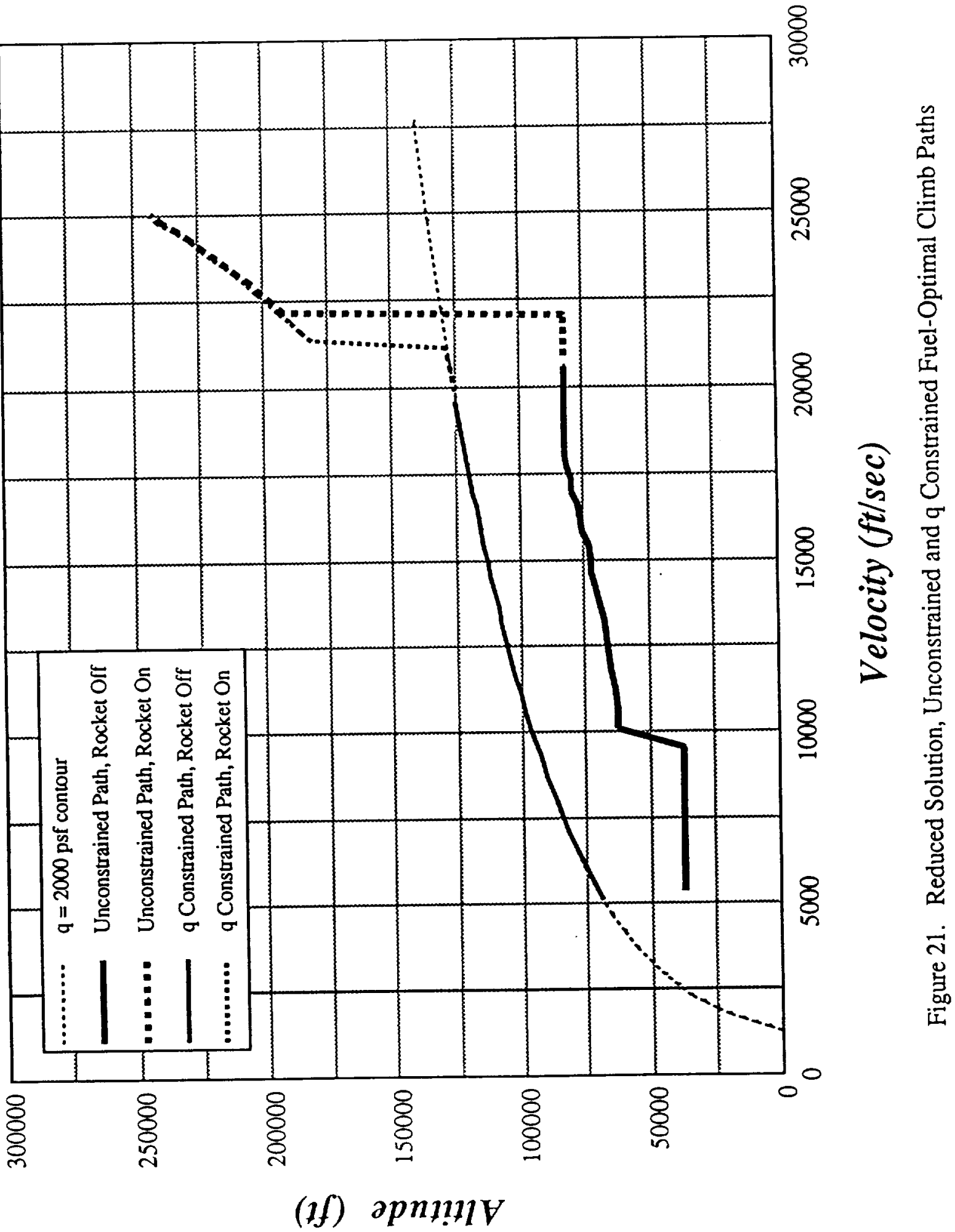




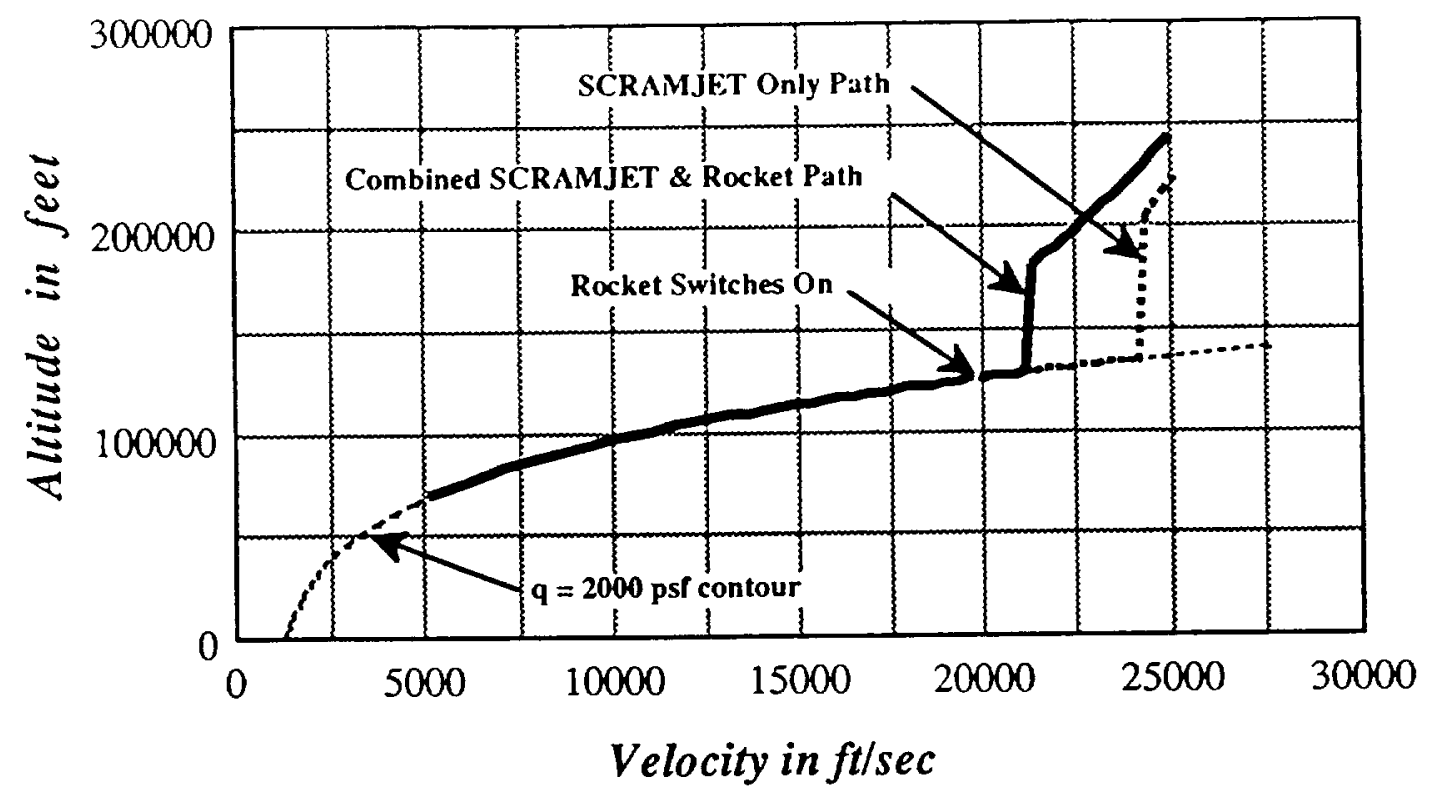

Figure 22. Reduced Solution, $q$ constrained fuel-optimal ascent trajectories.

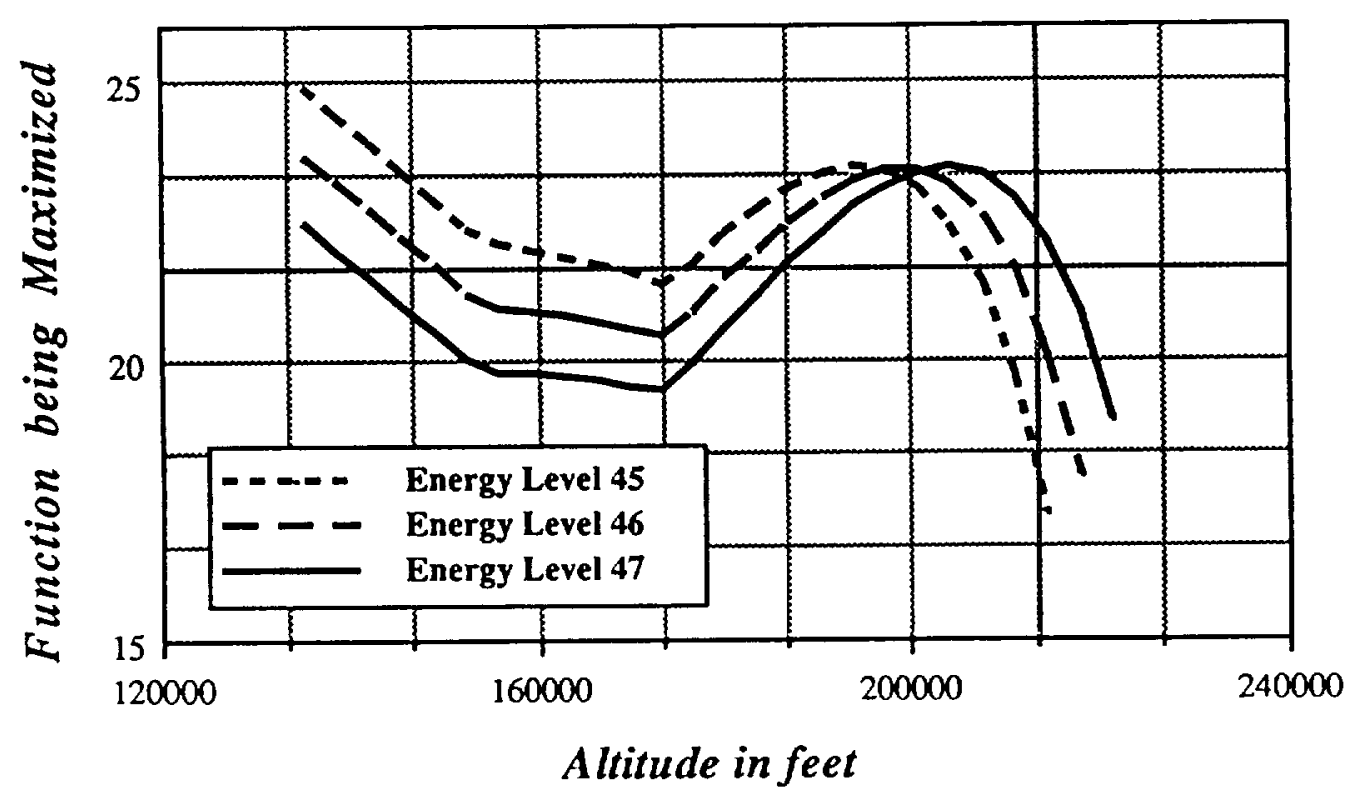

Figure 23. Reduced Solution, mechanism by which altitude discontinuity occurs for the q constrained case, SCRAMJET only. 


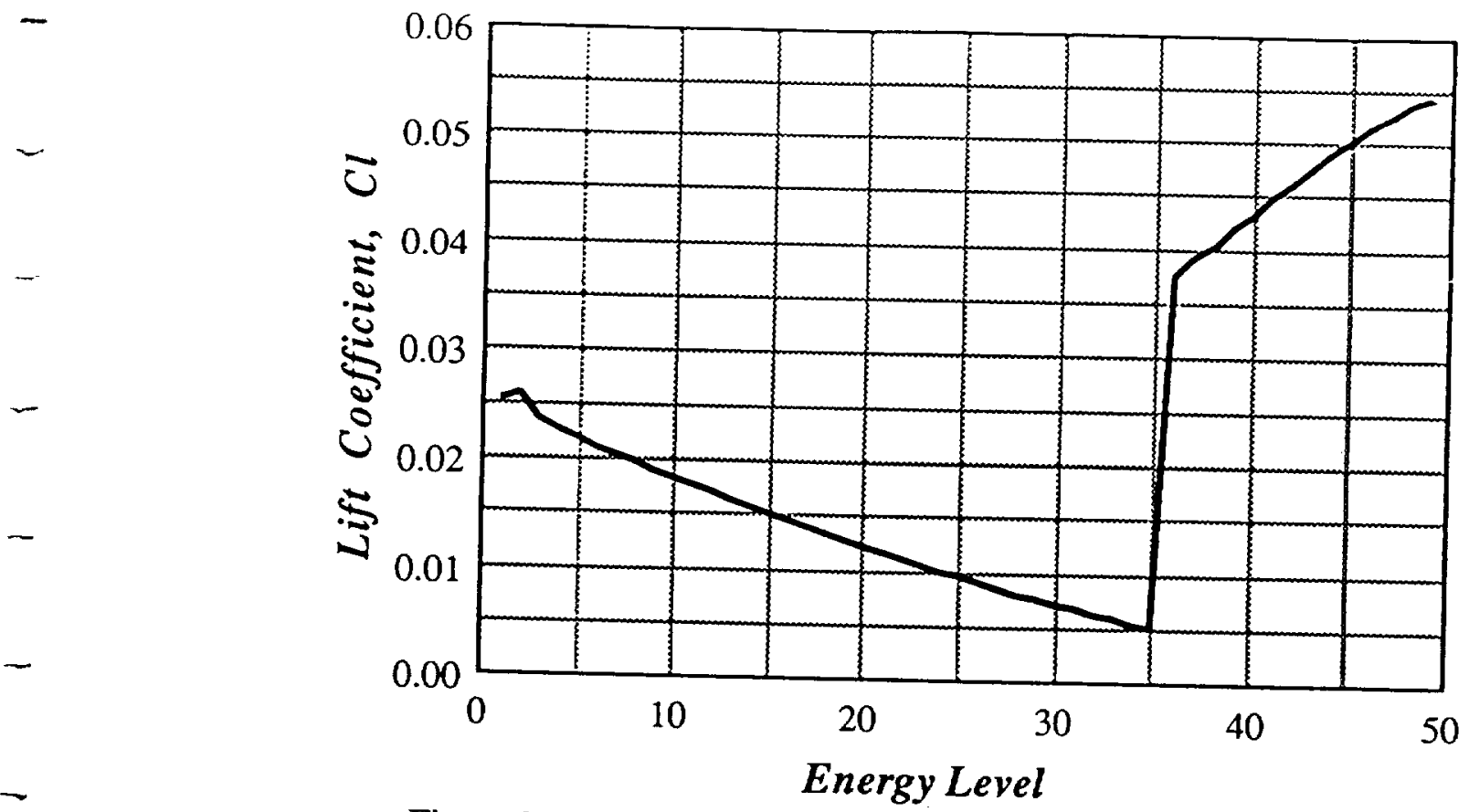
Figure 24. Reduced Solution, required lift coefficient computed along
the q constrained reduced solution.

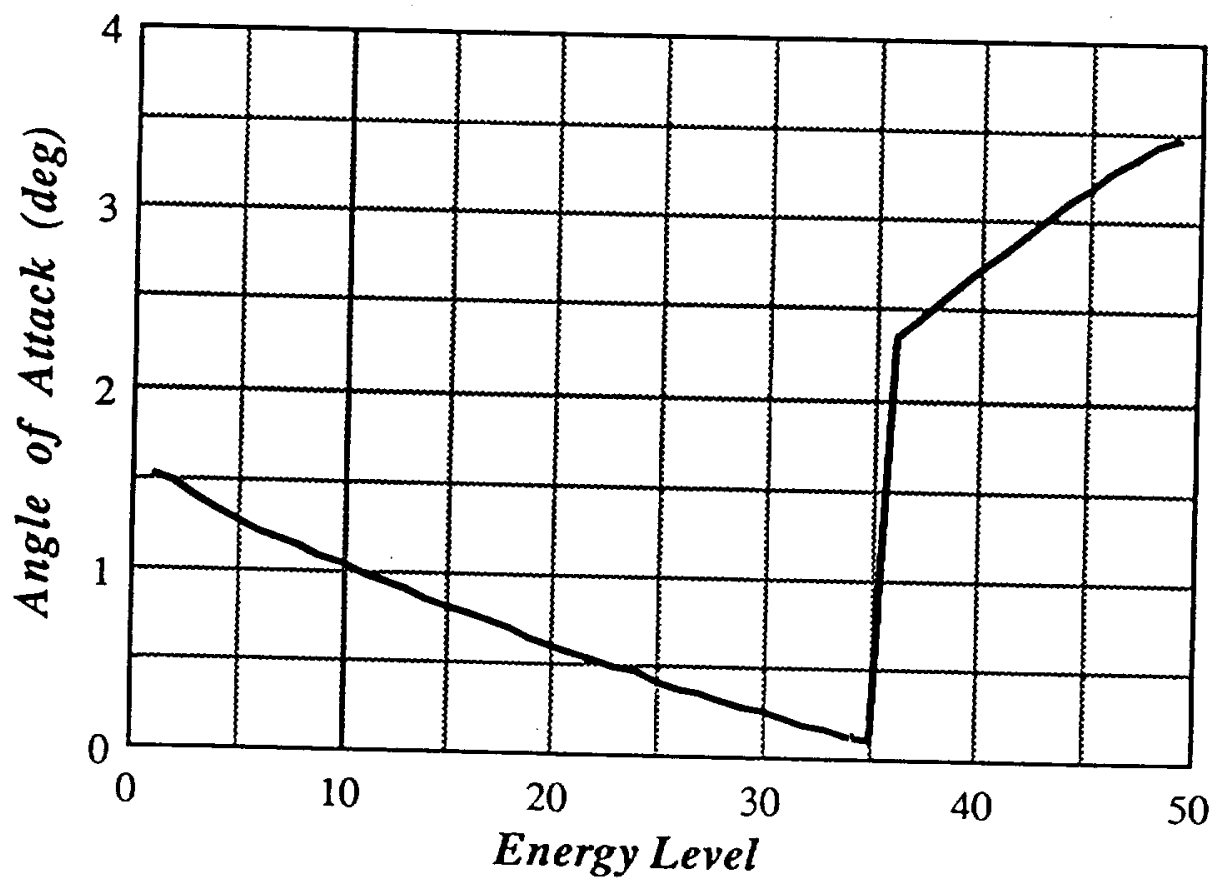

Figure 25. Reduced Solution, required angle of attack computed along the $q$ constrained reduced solution. 


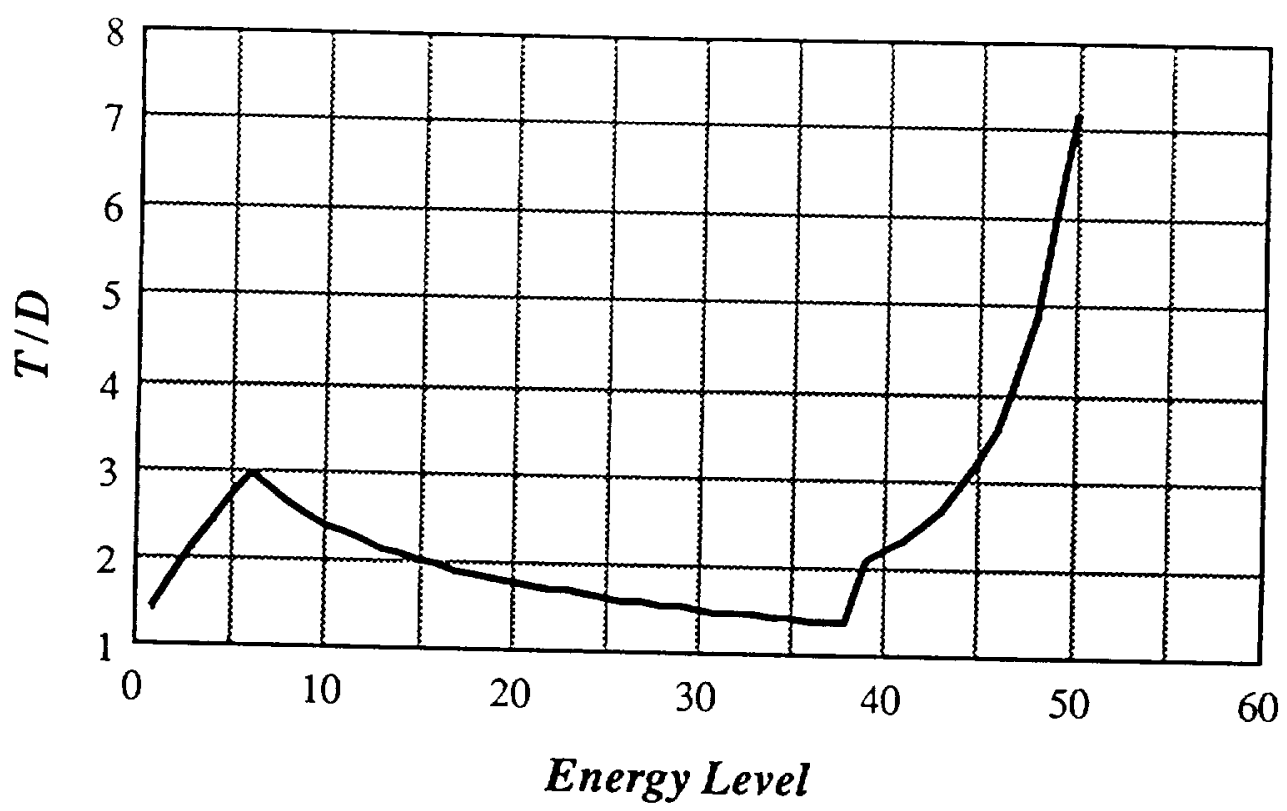

Figure 26. Reduced Solution, Thrust to Drag ratio computed along the unconstrained reduced solution.

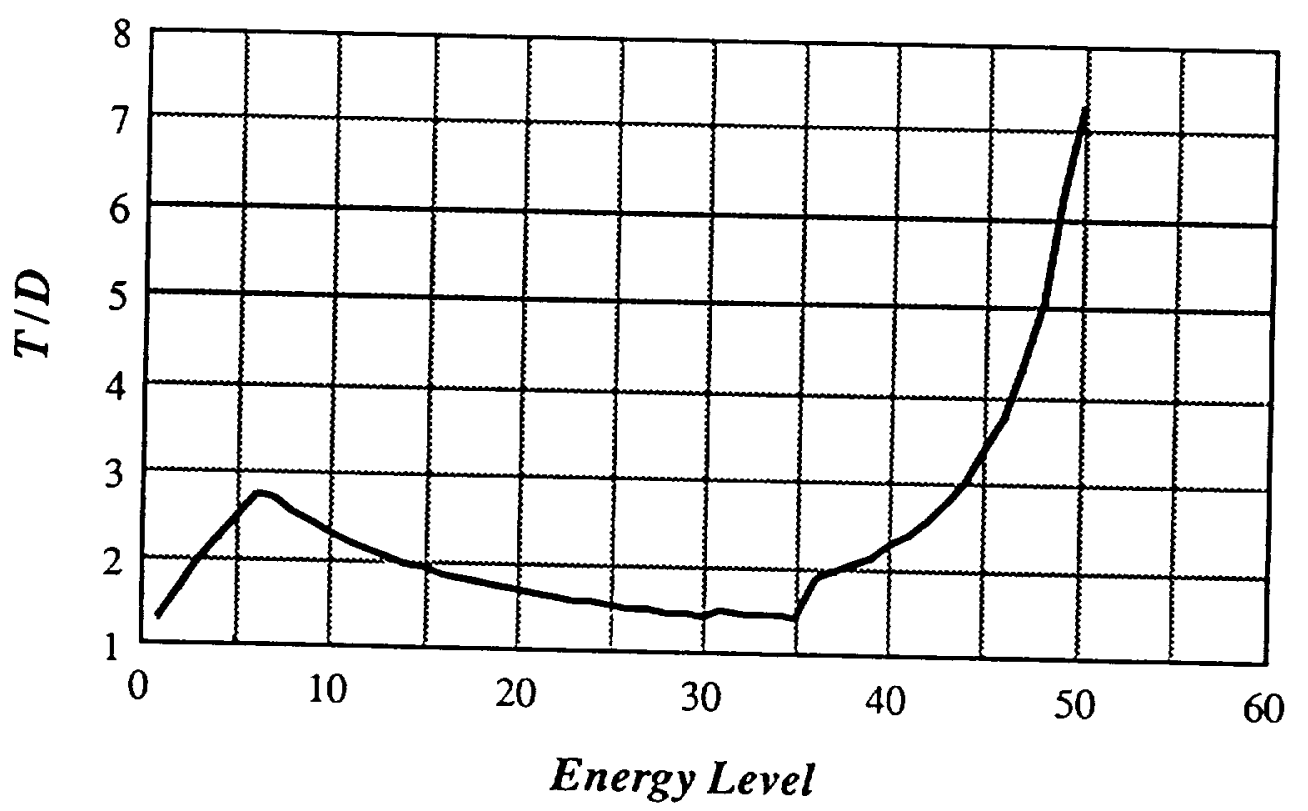
Figure 27 . Reduced Solution, Thrust to Drag ratio computed along the
q constrained reduced solution. 


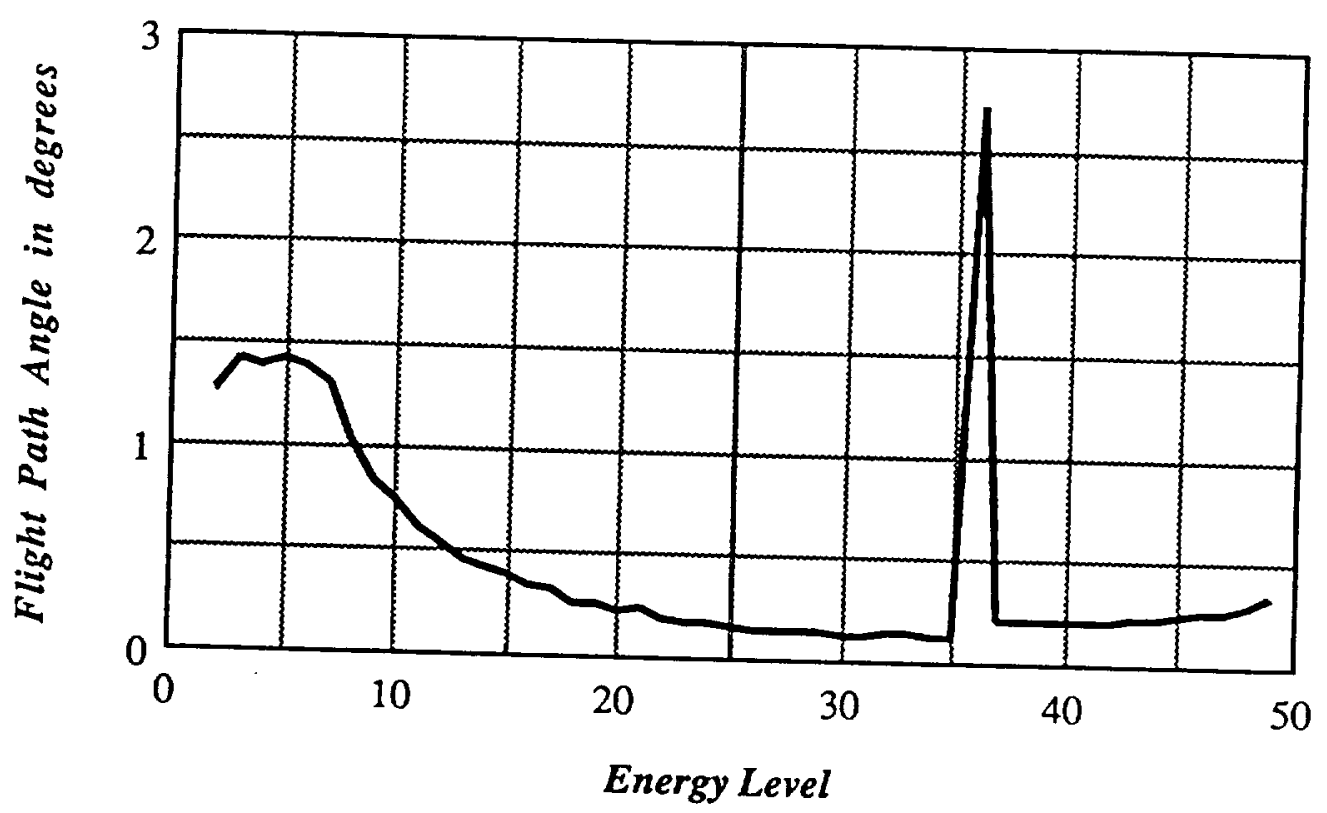

Figure 28. Reduced Solution, History of Flight Path Angle estimated along q constrained path, SCRAMJET/rocket case

\subsubsection{Boundary Layer Analysis}

\section{The Unconstrained Case}

The unconstrained boundary layer solution associated with equations (23-26) is obtained by introducing the time transformation $\tau=t / \varepsilon$ and again setting $\varepsilon=0$. The necessary conditions for optimality [56] become (where the prime notation denotes differentiation with respect to $\tau$ ):

$$
\begin{gathered}
\mathrm{H}_{\mathrm{BL}}=\lambda_{\mathrm{E}_{\mathrm{o}}} \mathrm{E}^{\prime}+\lambda_{\mathrm{m}_{\mathrm{o}}} \mathrm{m}^{\prime}+\lambda_{\mathrm{r}} \mathrm{V} \sin \gamma+\lambda_{\gamma} \gamma^{\prime}=0 \\
\mathrm{E}^{\prime}=0 \Rightarrow \mathrm{E}(\tau)=\mathrm{E}_{\mathrm{o}} \quad \lambda_{\mathrm{E}}=0 \Rightarrow \lambda_{\mathrm{E}}(\tau)=\lambda_{\mathrm{EO}_{\mathrm{o}}}
\end{gathered}
$$




$$
\begin{aligned}
\mathrm{m}^{\prime}=0 \Rightarrow \mathrm{m}(\tau) & =\mathrm{m}_{\mathrm{o}} \quad \lambda_{\mathrm{m}}^{\prime}=0 \Rightarrow \lambda_{\mathrm{m}}(\tau)=\lambda_{\mathrm{mo}} \\
\lambda_{\gamma}=-\frac{\partial \mathrm{H}_{\mathrm{BL}}}{\partial \gamma} \quad \lambda_{\mathrm{r}}=-\frac{\partial \mathrm{H}_{\mathrm{BL}}}{\partial \mathrm{r}} & \\
\mathrm{r}^{\prime} & =\mathrm{V} \sin \gamma \\
\gamma^{\prime} & =\frac{\mathrm{L}}{\mathrm{mV}}-\frac{\mu \cos \gamma}{\mathrm{Vr}^{2}}+\frac{\mathrm{V} \cos \gamma}{\mathrm{r}} \\
\frac{\partial \mathrm{H}_{\mathrm{BL}}}{\partial \mathrm{L}} & =0
\end{aligned}
$$

Evaluation of the partial derivative in (80) results in the following expression,

$$
\frac{\partial \mathrm{H}_{\mathrm{BL}}}{\partial \mathrm{L}}=\lambda_{\mathrm{E}_{\mathrm{o}}} \frac{\mathrm{V}}{\mathrm{m}}\left(\frac{-\mathrm{K}}{\mathrm{qs}}\right) 2 \mathrm{~L}+\lambda_{\gamma}\left(\frac{1}{\mathrm{mV}}\right)
$$

Substituting equation (81) into equation (80) and solving for $\mathrm{L}$ yields the following expression for optimal lift $\left(\mathrm{L}^{*}\right)$,

$$
\mathrm{L}^{*}=\lambda_{\gamma}\left[\mathrm{qs} / 2 \mathrm{KV}^{2} \lambda_{\mathrm{E}_{\mathrm{o}}}\right]
$$

The control solution for rocket throttle setting, $\eta$, remains the same as in the reduced solution (see equations 52).

The evaluation of $\lambda_{y}$ needed in equation (82) unfortunately requires the solution of a two-point boundary-value problem (TPBVP). The computational load associated with a TPBVP is to be avoided, and as a result we seek approximations to $\lambda_{\gamma}$ that yield an acceptable guidance law. 


\section{SP1 Control Solution}

When close to the reduced solution, it may be possible to use the expression for $\lambda_{\text {po }}$ given by equation (56) as an approximation to $\lambda_{\gamma}$ Substitution of this zeroth-order approximation into (82) results in the following simple feedback law,

$$
L^{*}=\left(\rho / \rho_{0}\right) L_{0}
$$

where $L_{0}$ is given by equation (33) and $\rho_{0}$ represents atmospheric density at $h=h_{0}$. Note that this solution is independent of the reduced solution costates and the final mass. For convenience this control law is referred to as the SP1 solution in the figures that follow. A numerical simulation using this control law to track a constant energy condition reveals, however, that this solution is unstable. This is clearly illustrated in Figures 29 and 30 where an altitude of 36,000 feet and zero flight path angle are to be tracked given an initial perturbation in altitude of 14,000 feet. This instability occurs for much smaller perturbations as well. Clearly we must seek a more suitable approximation for $\lambda_{\gamma}$.

\section{SP2 Control Solution}

Expansion of the boundary layer necessary conditions to first order about the reduced solution, which is an equilibrium point of these equations, results in the following linear perturbation equations [57-60]:

$$
\left[\begin{array}{c}
\delta r^{\prime} \\
\delta \gamma^{\prime} \\
\delta \lambda_{\mathrm{r}}^{\prime} \\
\delta \lambda_{\gamma^{\prime}}
\end{array}\right]=\left[\begin{array}{cccc}
0 & \mathrm{v}_{0} & 0 & 0 \\
\mathrm{~A}_{21} & 0 & 0 & \mathrm{~A}_{24} \\
\mathrm{~A}_{31} & 0 & 0 & -\mathrm{A}_{21} \\
0 & \mathrm{~A}_{42} & -\mathrm{V}_{0} & 0
\end{array}\right]\left[\begin{array}{c}
\delta \mathrm{r} \\
\delta \gamma \\
\delta \lambda_{\mathrm{r}} \\
\delta \lambda_{\gamma}
\end{array}\right]
$$




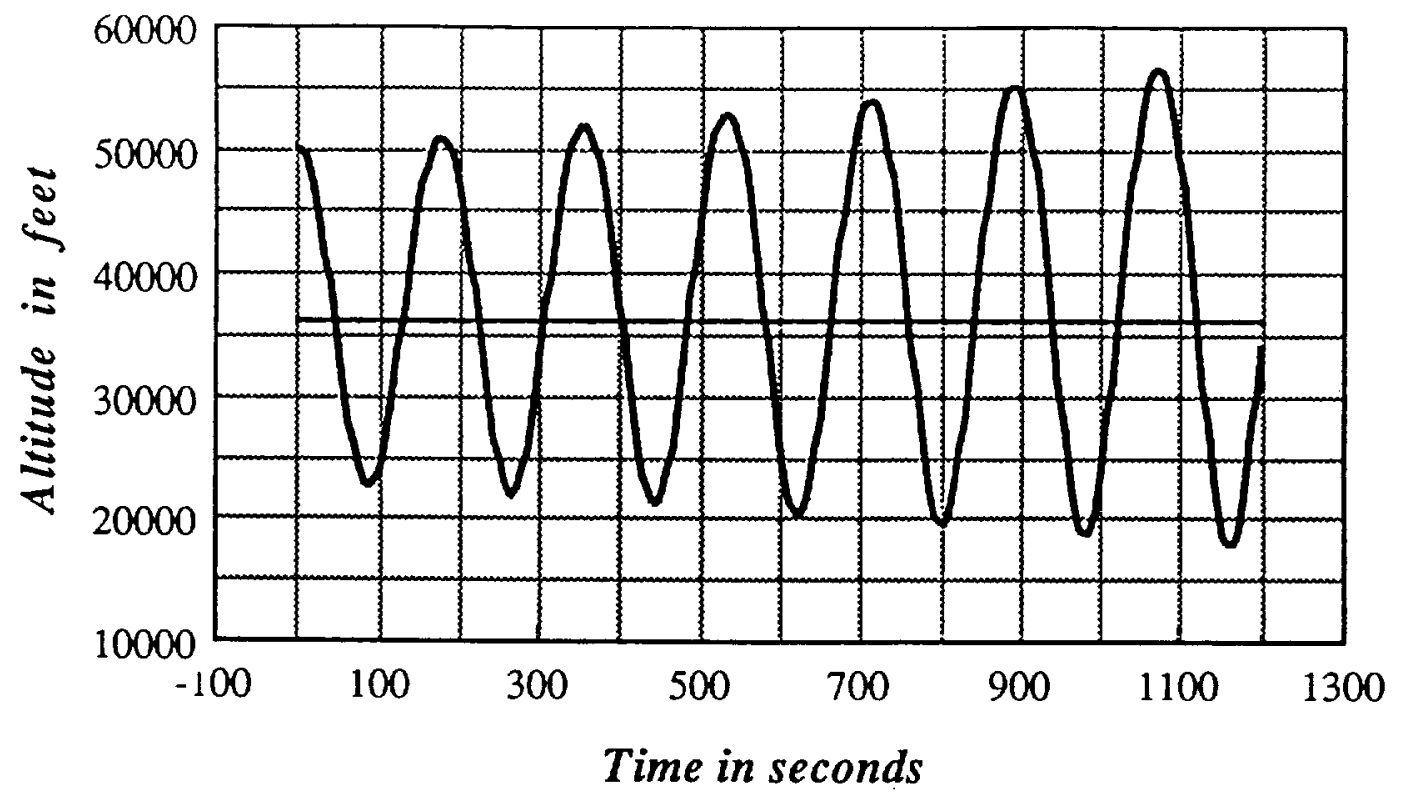

Figure 29. SP1 Controller Tracking Constant Energy Reduced Solution, Altitude versus Time

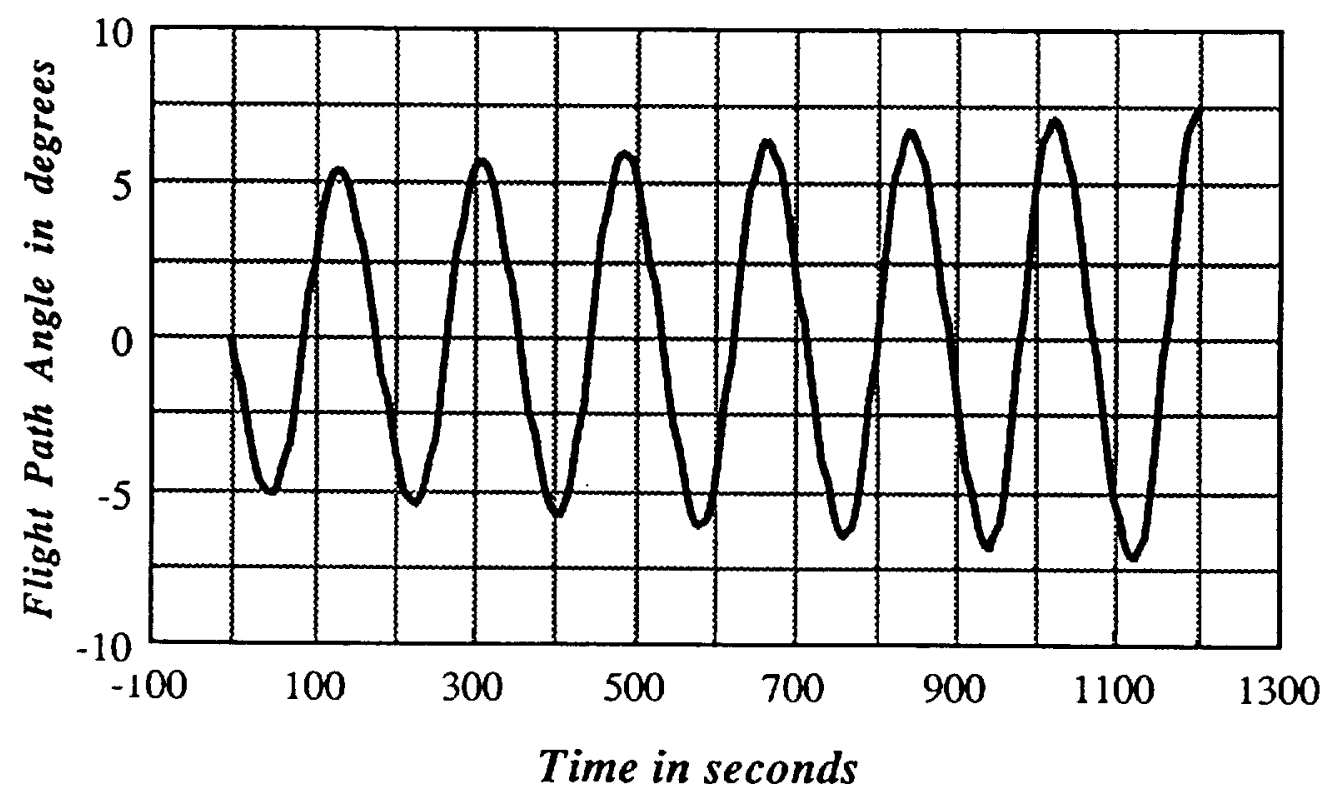

Figure 30. SP1 Controller Tracking Constant Energy Reduced Solution, Flight Path Angle versus Time 
The square matrix in equation (84) is a Hamiltonian matrix, and all of its terms but $A_{31}$ can readily be evaluated analytically.

$$
\begin{aligned}
& A_{21}=\left[\frac{L_{0}}{m \rho_{0} V_{0}}\right] \frac{\partial \rho}{\partial r_{0}}-\frac{V_{0}}{r_{0}^{2}} \\
& A_{24}=\frac{q_{0} s}{2 K m V_{0}^{3} \lambda_{E_{0}}} \\
& A_{31}=-\left.\frac{\partial^{2} \mathrm{H}_{B L}^{*}}{\partial r^{2}}\right|_{0} \\
& A_{42}=-\lambda_{\gamma_{0}}\left[\frac{\mu}{V_{0} r_{0}^{2}}-\frac{V_{0}}{r_{0}}\right]
\end{aligned}
$$

where $\mathrm{H}^{*} \mathrm{BL}$ is defined as in equation (74), but after elimination of the lift control using (82). The rocket throttle control is completely determined by the switching condition given in equation (52) and thus may be treated as a known constant. The right hand side of equation (87) can be evaluated numerically as follows. Consider a Taylor's series expansion of (74) to second order

$$
\mathrm{H}_{\mathrm{BL}}^{*}\left(\mathrm{~h}_{\mathrm{o}}+\delta \mathrm{h}\right)=\mathrm{H}_{\mathrm{BL}}^{*}+\frac{\partial \mathrm{H}_{\mathrm{BL}}^{*}}{\partial \mathrm{h}} \delta \mathrm{h}+\frac{\partial^{2} \mathrm{H}_{\mathrm{BL}}^{*}}{\partial \mathrm{h}^{2}} \frac{\delta h^{2}}{2}+\ldots
$$

Taking into account the fact that $\mathrm{H}^{*}{ }_{\mathrm{BL}}$ is zero along the optimal path and simplifying the notation we have

$$
\mathrm{H}_{\mathrm{BL}}^{*}=\mathrm{H}_{\mathrm{h}} \delta \mathrm{h}+\mathrm{H}_{\mathrm{hh}} \delta \mathrm{h}^{2} R
$$

Define forward and backward perturbations with respect to $h$ about $h_{0}$, denoted + and respectively, as 


$$
\begin{aligned}
& \mathrm{H}^{+}=\mathrm{H}_{\mathrm{h}} \delta \mathrm{h}+\mathrm{H}_{\mathrm{hh}} \delta \mathrm{h}^{2} / 2 \\
& \mathrm{H}^{-}=-\mathrm{H}_{\mathrm{h}} \delta \mathrm{h}+\mathrm{H}_{\mathrm{hh}} \delta \mathrm{h}^{2} / 2
\end{aligned}
$$

Adding together $\mathrm{H}^{+}$and $\mathrm{H}^{-}$and then solving for the desired quantity we arrive at the following numerical estimate

$$
\frac{\partial^{2} \mathrm{H}_{\mathrm{BL}}^{*}}{\partial \mathrm{r}^{2}}=\mathrm{H}_{\mathrm{hh}}=\left(\mathrm{H}^{+}+\mathrm{H}^{-}\right) / \delta \mathrm{h}^{2}
$$

A well-known property of Hamiltonian Matrices is that their eigenvalues are arranged symmetrically about the imaginary axis. Since we require that the boundary layer dynamics be stable forward in time, the state vector in (84) is expressed as,

$$
\begin{aligned}
& \vec{x}=k_{1} \vec{a}+k_{2} \vec{b} \\
& \vec{x}^{T}=\left[\delta r, \delta \gamma, \delta \lambda_{r}, \delta \lambda_{\gamma}\right]
\end{aligned}
$$

where

and $\vec{a}$ and $\vec{b}$ are the real and imaginary parts of the eigenvectors associated with the stable eigenvalues. At any given time the state perturbations away from the reduced solution, $\delta r$ and $\delta \gamma$, are known. The scalars $k_{1}$ and $k_{2}$ are unknown, as are the perturbations $\delta \lambda_{r}$ and $\delta \lambda_{y}$. Thus, equation (94) constitutes a system of four scalar equations in four unknowns. Use of equation (94) to pick the free values of $\delta \lambda_{r}$ and $\delta \lambda_{y}$ allows the suppression of the unstable modes. That is, we allow only initial conditions that lie in the subspace spanned by the eigenvectors associated with the stable eigenvalues. To solve for $k_{1}$ and $k_{2}$, partition equation (94) into upper, (U), and lower, (L), systems as follows,

$$
\left[\begin{array}{l}
\vec{x}_{U} \\
\vec{x}_{L}
\end{array}\right]=k_{1}\left[\begin{array}{l}
\vec{a}_{U} \\
\vec{a}_{L}
\end{array}\right]+k_{2}\left[\begin{array}{l}
\vec{b}_{U} \\
\vec{b}_{L}
\end{array}\right]
$$

Now since $\delta r$ and $\delta \gamma$ are known, we can solve the upper system for $k_{1}$ and $k_{2}$ as follows, 


$$
\overrightarrow{\mathrm{x}}_{U}=\left[\begin{array}{l}
\delta r \\
\delta \gamma
\end{array}\right]=\mathrm{k}_{1} \overrightarrow{\mathrm{a}}_{U}+\mathrm{k}_{2} \overrightarrow{\mathrm{b}}_{U}
$$

or

$$
\left[\begin{array}{l}
\mathrm{k}_{1} \\
\mathrm{k}_{2}
\end{array}\right]=\left[\overrightarrow{\mathrm{a}}_{\mathrm{U}} \overrightarrow{\mathrm{b}}_{\mathrm{U}}\right]^{-1}\left[\begin{array}{l}
\delta \mathrm{r} \\
\delta \gamma
\end{array}\right]
$$

With $\mathrm{k}_{1}$ and $\mathrm{k}_{2}$ known, it is a simple matter to calculate $\delta \lambda_{\mathrm{I}}$ and $\delta \lambda_{\gamma}$ using the lower system,

$$
\vec{x}_{L}=\left[\begin{array}{l}
\delta \lambda_{r} \\
\delta \lambda_{\gamma}
\end{array}\right]=\left[\begin{array}{ll}
\vec{a}_{L} & \vec{b}_{L}
\end{array}\right]\left[\begin{array}{l}
k_{1} \\
k_{2}
\end{array}\right]
$$

Now $L^{*}$ can be evaluated using equation (82) where

$$
\lambda_{\gamma}=\lambda_{\gamma_{0}}+\delta \lambda_{\gamma}
$$

That is,

$$
\mathrm{L}^{*}=\left(\lambda_{\gamma_{0}}+\delta \lambda_{\gamma}\right)\left[\mathrm{qs} / 2 \mathrm{KV}^{2} \lambda_{\mathrm{E}_{o}}\right]
$$

For convenience, this control law will be referred to as SP2 in the figures that follow.

For the vehicle model being considered, the stable eigenvalues of (84) at the starting energy level are $(-0.2573 \pm \mathrm{j} 0.2599)$. These eigenvalues corresponds to a natural frequency $\left(\omega_{n}\right)$, damped natural frequency $\left(\omega_{d}\right)$, time period $(T)$, damping ratio $(\zeta)$ and $2 \%$ settling time $\left(\mathrm{T}_{\mathrm{s}}\right)$ of:

$$
\begin{array}{ll}
\omega_{\mathrm{n}}=.3657 \mathrm{rad} / \mathrm{sec} & \mathrm{T}=24.18 \mathrm{sec} \\
\omega_{\mathrm{d}}=.2599 \mathrm{rad} / \mathrm{sec} & \mathrm{T}_{\mathrm{s}}(2 \%)=15.54 \mathrm{sec}
\end{array}
$$


Figures 31 and 32 present altitude and flight path angle time histories generated using the control law (SP2) presented as equation (101). Excellent behavior, as predicted by (102), is evident in tracking the same constant energy conditions as in the previous Figures 27 and 28. Difficulties arise, however, in that very large lift values (i.e. proportional to the altitude perturbation, which at 14,000 feet is fairly large) are commanded. If a lift limit is enforced, the performance of the SP2 controller is severely degraded. This condition is depicted in Figures 33 and 34 which again present altitude and flight path angle time histories for the same initial conditions, but with the magnitude of the SP2 commanded lift constrained so that $\mathrm{L} / \mathrm{W} \leq 2.0$. The corresponding lift control time histories for the unconstrained and control constrained cases are presented as Figures 35 and 36, respectively. For the given altitude perturbation, the lift limited SP2 controller generates a 20,000 foot undershoot. Clearly the lift limited SP2 control law is only suitable for small perturbations about the reduced solution. Configured as a launch vehicle, it is indeed reasonable to assume that the aerospace plane would not deviate far from the reduced solution climb path during ascent to orbit. However, two major stumbling blocks remain. Number one is the requirement that the vehicle traverse the large interior altitude discontinuity present in the unconstrained (and the dynamic pressure constrained) reduced solution climb path (see Figure 21 on page 56 ). This discontinuity introduces a very large altitude perturbation and would have to be traversed using an alternate controller. It has been shown that such problems can be addressed by constructing interior boundary layers $[61,62]$, but again we are left with having to solve a TPBVP on line. Introduction of an aerodynamic heating constraint may eliminate the altitude discontinuity from the reduced solution, but will still introduce a rapid change in slope in the climb path at the juncture of the path with the constraint boundary.

The second major stumbling block is the requirement for mission abort capability. Initiation of an abort maneuver may radically change the reduced solution and as a result the vehicle state may initially lie far from the reduced solution path. Again the approach of linearizing the boundary layer necessary conditions about the reduced solution may produce an unsuitable controller.

In review, the SP1 and SP2 lift control solutions presented above were attempts to form a suitable approximation to the costate $\lambda_{\gamma}$ that is needed in equation (82) in order to alleviate having to solve a TPBVP. Note that control dependence on the final mass, 


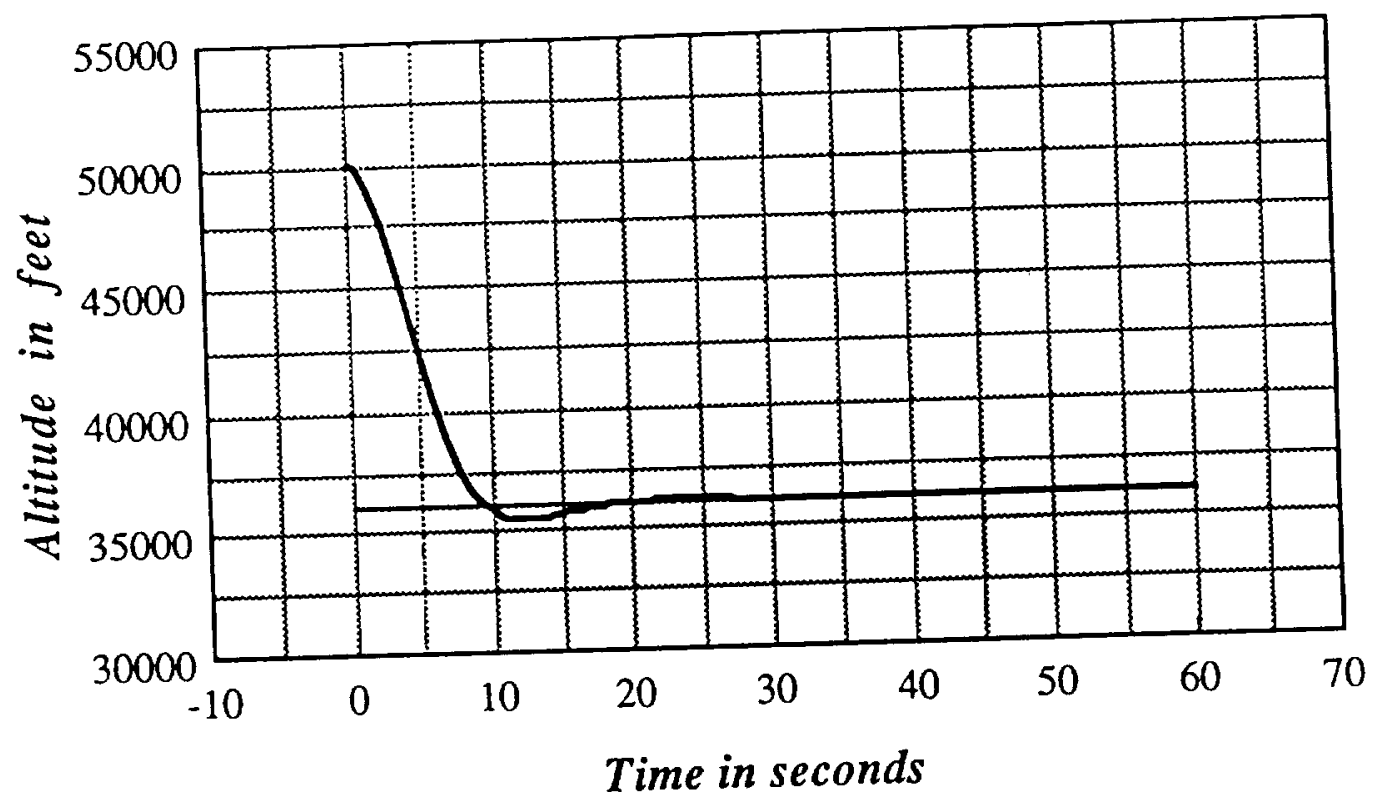

Figure 31. SP2 Controller Tracking Constant Energy Reduced Solution, Altitude versus Time

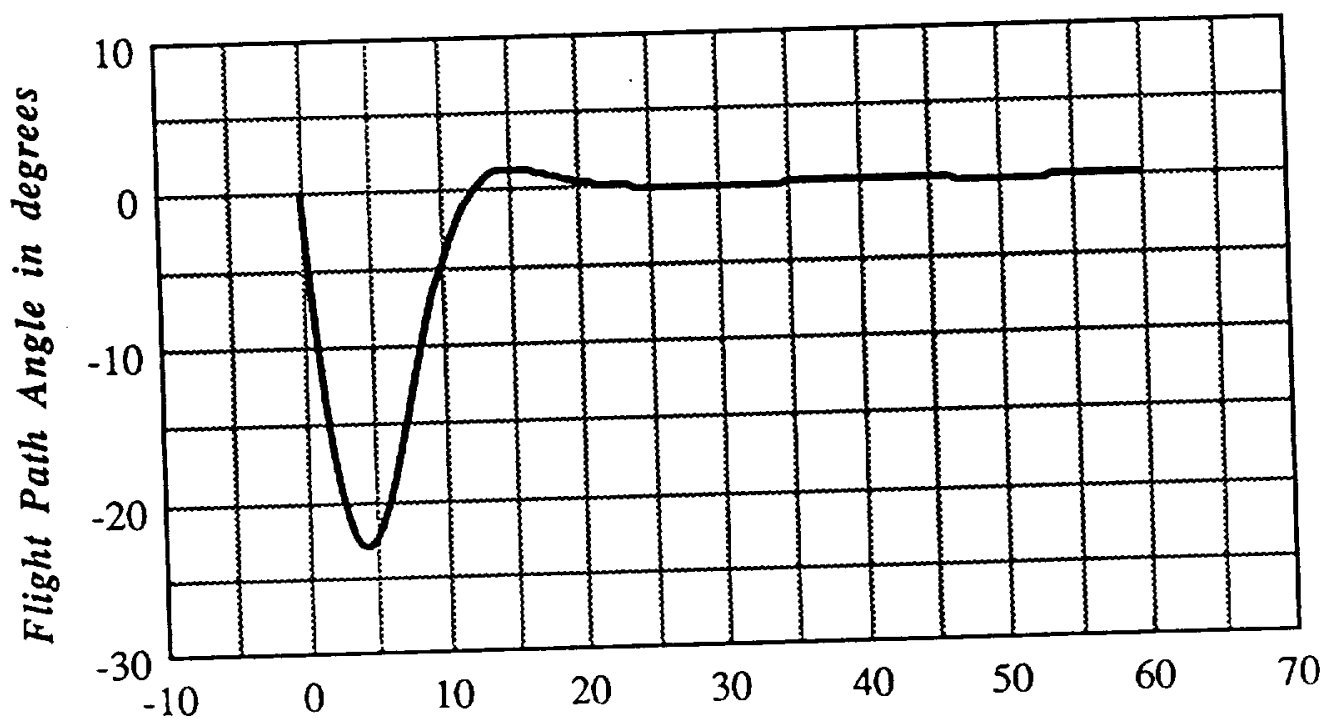

Time in seconds

Figure 32. SP2 Controller Tracking Constant Energy Reduced Solution, Flight Path Angle versus Time 


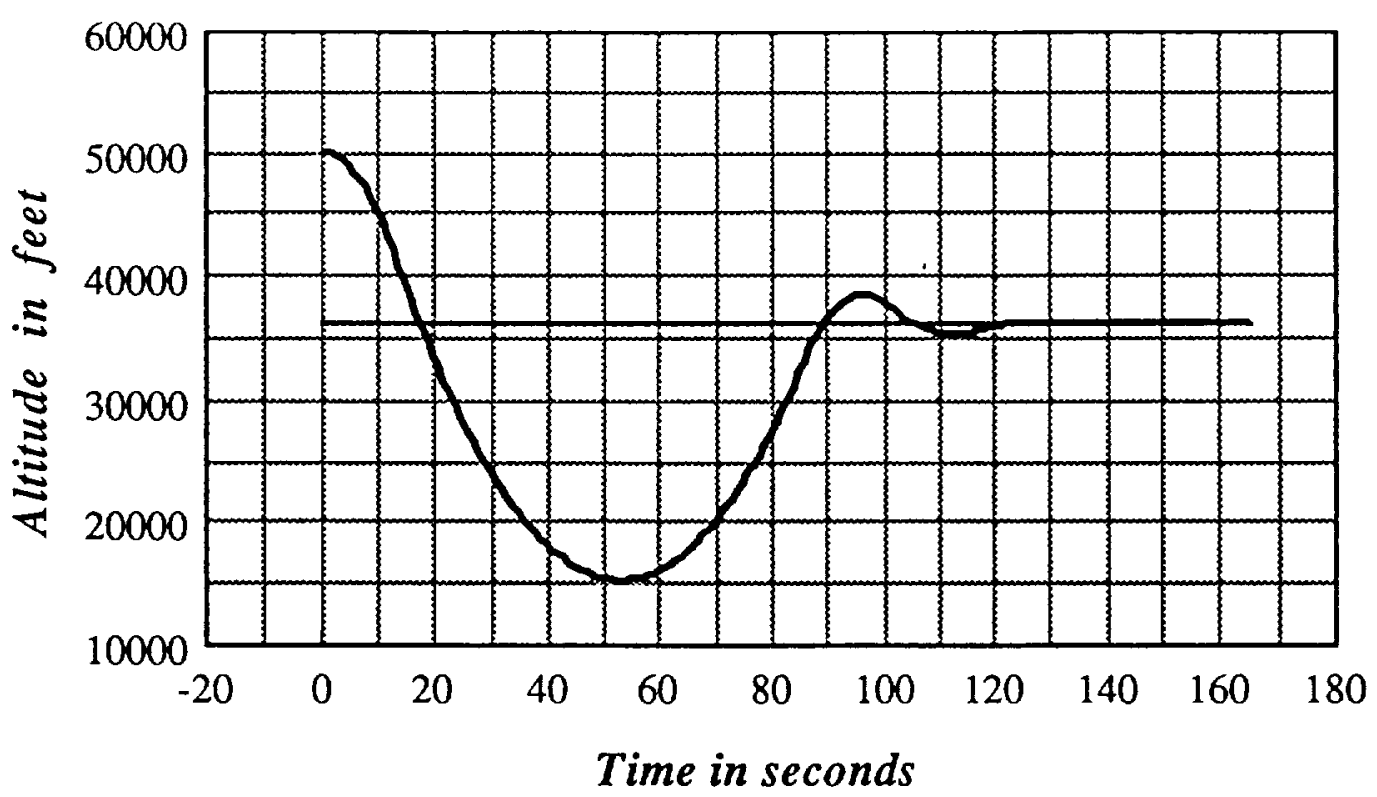

Figure 33. SP2 Controller Tracking Constant Energy Reduced Solution, Altitude versus Time, $\mathrm{L} / \mathrm{W} \leq 2.0$

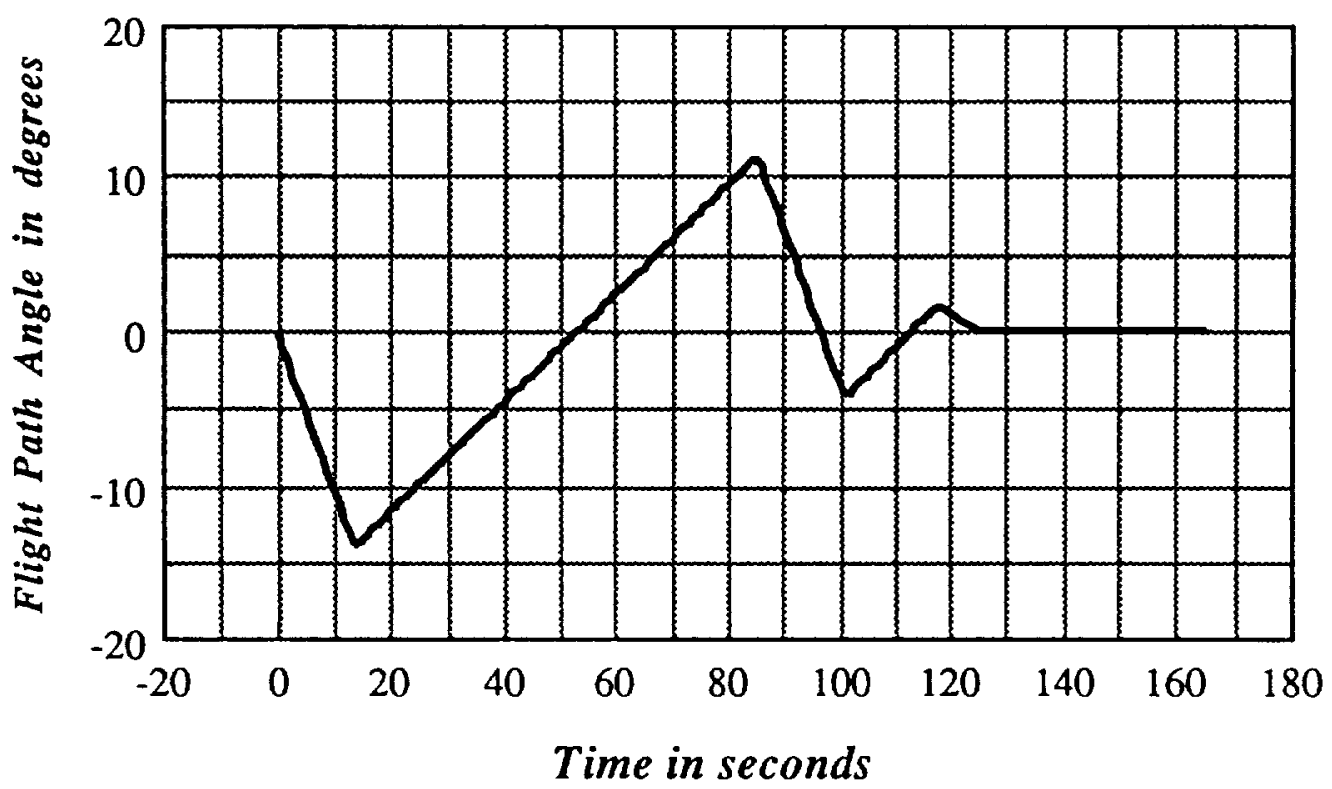

Figure 34. SP2 Controller Tracking Constant Energy Reduced Solution, Fligth Path Angle versus Time, $\mathrm{L} / \mathrm{W} \leq 2.0$ 


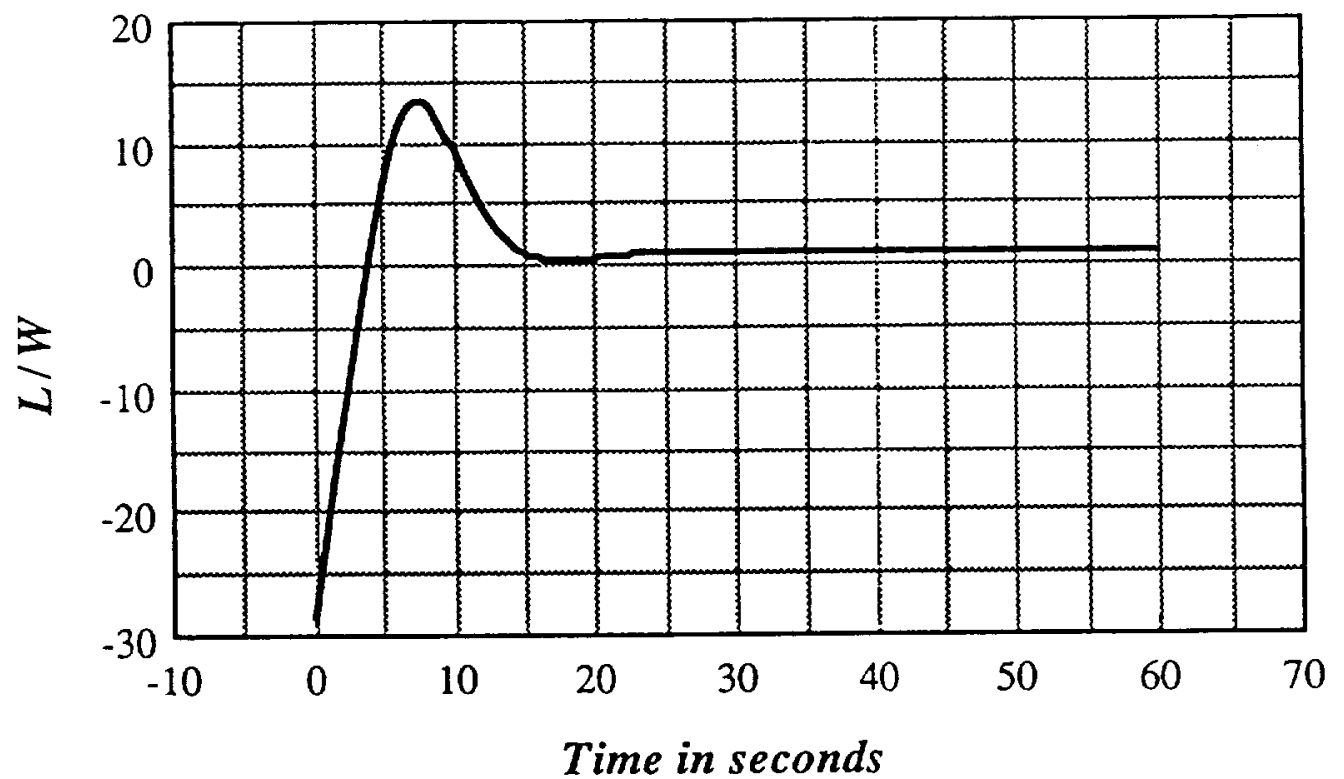

Figure 35. L/W for SP2 Controller Tracking Constant Energy Reduced Solution (see corresponding Figures 31 and 32)

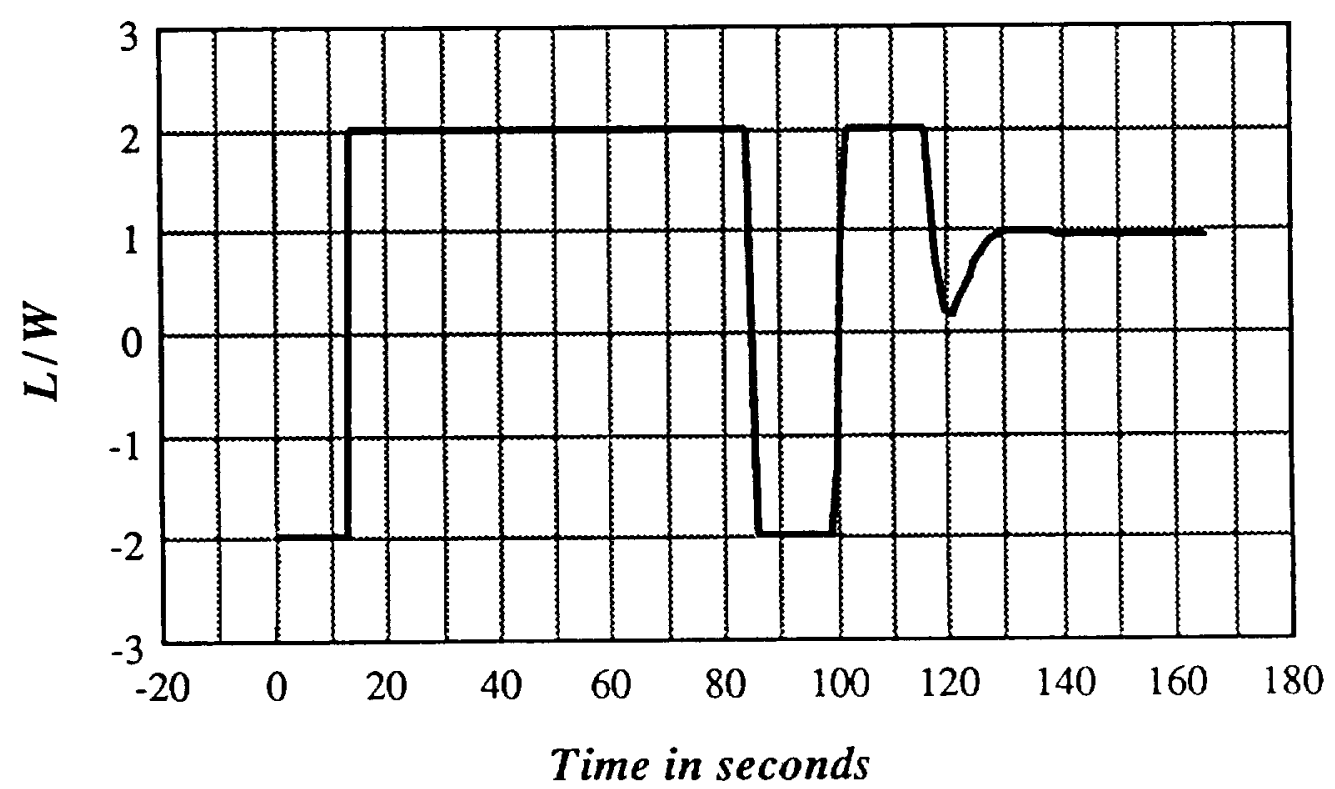

Figure 36. L/W for SP2 Controller Tracking Constant Energy Reduced Solution, (see corresponding Figures 33 and 34) 
although weak, is introduced through $\lambda \gamma_{\mathrm{o}}$ (see 56,57 and 60) in both SP1 and SP2. Recall that $\delta \lambda_{\gamma}$ is determined in the SP2 control solution by linearization of the boundary layer necessary conditions about the reduced solution and that it has been shown that the resulting controller, when lift limited, is suitable only for small perturbations. As noted in a later subsection, state constraints in the boundary layer further complicate this linearization technique. It is suspected that in order to avoid the excessive lift commands that plague this linearized approach on must capture the full nonlinearites of the problem. The forced separation of altitude and flight path angle dynamics and a suitable penalty on flight path angle in the performance index as in reference [40] may yield a more accurate controller. It is proposed in Section 7 that this avenue be explored. Another problem cited in using the initial boundary layer lift control solution as the control law all along the trajectory is the lack of ability on the part of the controller to anticipate rapid changes in altitude; it can only react to such changes once they are encountered. In the next subsection of this report still another approach to constructing a lift control solution, admittedly suboptimal, is employed and shown to alleviate some of the afore mentioned problems.

\section{NLT Control Solution}

As an alternative approach to tracking the reduced solution, a nonlinear transformation (NLT) technique is employed as follows [63-68].

Again consider the altitude and flight path angle dynamics:

$$
\begin{aligned}
& \dot{r}=V \sin \gamma \\
& \dot{\gamma}=\frac{L}{\mathrm{mV}}-\frac{\mu \cos \gamma}{\mathrm{Vr}^{2}}+\frac{\mathrm{V} \cos \gamma}{\mathrm{r}}
\end{aligned}
$$

Note that we have system equations in block triangular form, that is of the form

$$
\begin{aligned}
& \dot{x}_{1}=\mathrm{f}\left(\mathrm{x}_{1}, \mathrm{x}_{2}\right) \\
& \dot{x_{2}}=\mathrm{g}\left(\mathrm{x}_{1}, \mathrm{x}_{2}, \mathrm{u}\right)
\end{aligned}
$$


where $\mathrm{x}_{1}=\mathrm{r}, \mathrm{x}_{2}=\gamma$, and $\mathrm{u}=\mathrm{L}$. Next we take successive total time derivatives of $\mathrm{r}$ until explicit dependence on the control appears.

$$
\ddot{r}=-\frac{\mu \sin ^{2} \gamma}{r^{2}}+\frac{L \cos \gamma}{m}-\frac{\mu \cos ^{2} \gamma}{r^{2}}+\frac{V^{2} \cos ^{2} \gamma}{r}
$$

Since the control, $\mathrm{L}$, appears in the second time derivative we define $\mathrm{U}$, the peusdo control, as

$$
\mathrm{U}=\ddot{\mathrm{r}}
$$

It is desired that $U$ be determined as follows

$$
U=K_{p}\left(r_{o}-r\right)+K_{d}\left(\dot{r}_{o}-\dot{r}\right)
$$

where $r_{0}$ denotes the reduced solution radius at the current energy level and the time derivative of $r_{0}$ denotes the climb rate required to stay on the reduced solution as energy is gained. This climb rate can be estimated by defining an appropriate increment in energy, evaluating the reduced solution at this higher energy level and then estimating the required climb rate using a forwards difference.

The inverse transformation is defined by solving for $\mathrm{L}$ in (108) using (107) and (109),

$$
\mathrm{L}=\left[\mathrm{U}+\frac{\mu}{\mathrm{r}^{2}}-\frac{\mathrm{V}^{2} \cos ^{2} \gamma}{\mathrm{r}}\right] \frac{\mathrm{m}}{\cos \gamma}
$$

This lift control solution is referred to as the NLT control solution in the figures that follow. Note that as $r$ and $\gamma$ approach their reduced solution values equation (110) approaches the reduced solution value of lift given by equation (33). A block diagram depicting the conceptual implementation of the nonlinear transformation technique to yield the controller defined by equation (110) is presented in Figure 37. This is mathematically 
equivilent to the linear system depicted in Figure 38 which is used to design the controller. The corresponding closed loop transfer function is

$$
G(s)=\frac{K_{d} s+K_{p}}{s^{2}+K_{d} s+K_{p}}
$$

where the gains $K_{p}$ and $K_{d}$ for the second order system can be written in terms of the damping ratio, $\zeta$, and natural frequency, $\omega_{n}$, as

$$
\begin{aligned}
& K_{p}=\omega_{n}^{2} \\
& K_{d}=2 \zeta \omega_{n}
\end{aligned}
$$

The performance of this controller can be dictated by selecting the values of $K_{p}$ and $K_{d}$ to yield the desired dynamic response.

Figures 39 and 40 present the altitude and flight path angle time histories generated using the NLT controller to track the same constant energy conditions as before: $h_{o}=$ 36,000 feet, $\gamma_{o}=0$. The gains were selected so as to match the performance of the SP2 controller at the same energy level. As expected, the trajectories and the corresponding lift control (Figure 43) are nearly identical to those of the SP2 controller that were given in Figures 31, 32 and 35. Figures 41 and 42 present the altitude and flight path angle histories for the case in which the magnitude of the NLT lift control is constrained so that $L / W \leq$ 2.0 , for the same gains and for the same initial conditions as for the SP2 controller. The corresponding lift control is shown in Figure 44. As in the unconstrained case, the NLT controller very nearly duplicates the behavior of the SP2 controller.

Figures 45, 46 and 47 present the case in which the NLT controller gains are modified to yield a $2 \%$ settling time of 120.0 seconds rather than the 15.5 seconds demanded by SP2. The resulting trajectories are well behaved. More importantly, $L / W$ remains positive and less than 1.2. The NLT controller clearly provides a simple means for adjusting its performance to yield a control solution within reasonable bounds. It also provides guaranteed stability properties which the SP2 solution lacks given large perturbations. 

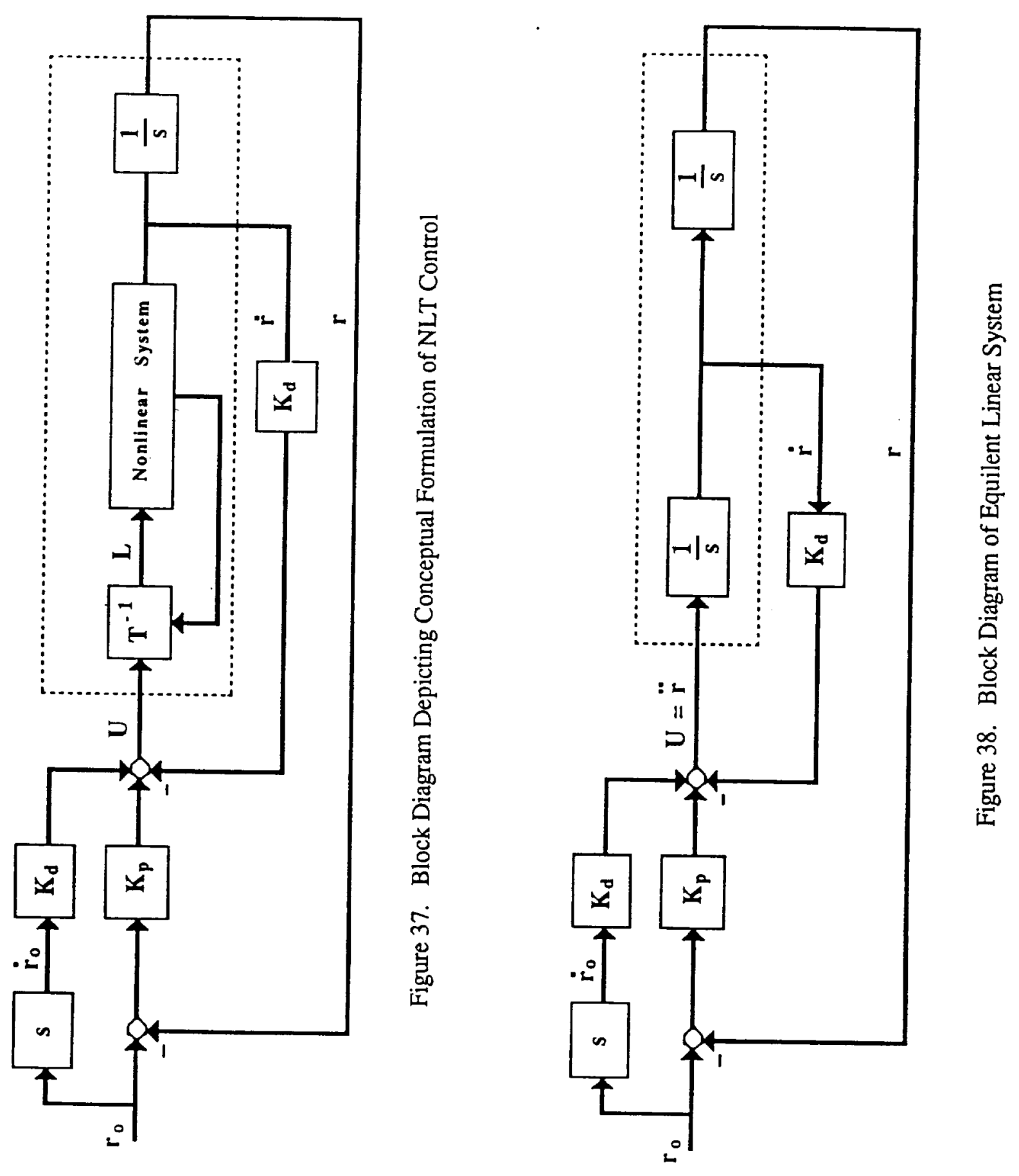


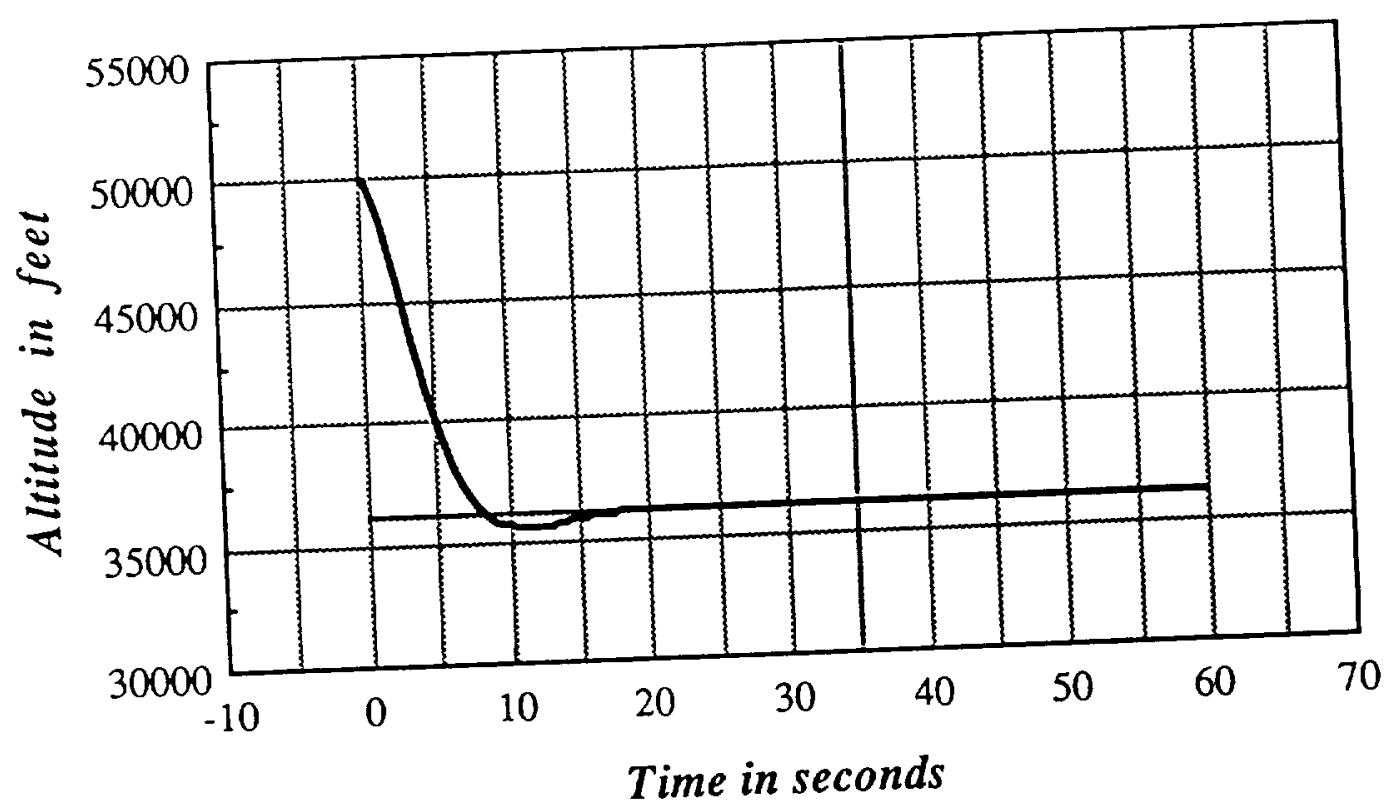

Figure 39. NLT Controller Tracking Constant Energy Reduced Solution, Altitude versus Time, Gains selected to match SP2 (see corresponding Figures 31 and 32)

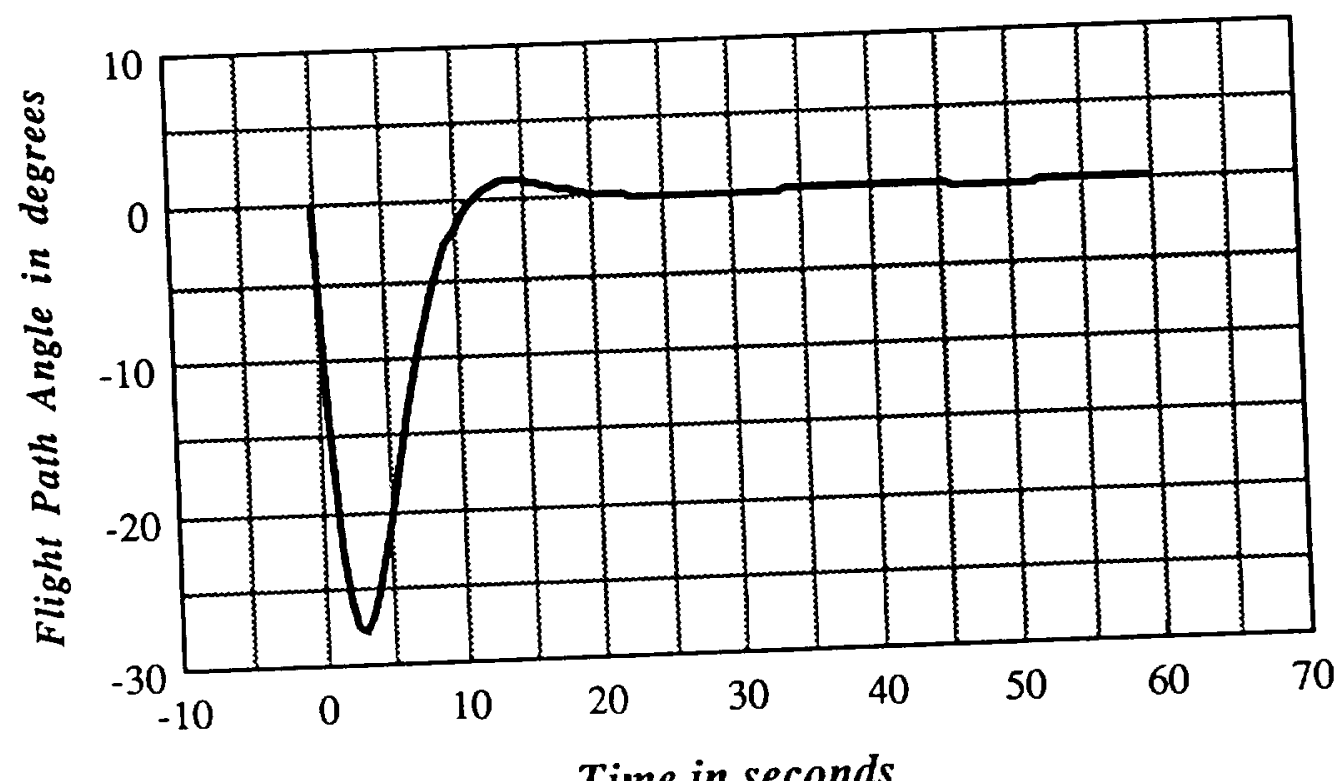

Figure 40. NLT Controller Tracking Constant Energy Reduced Solution, Flight Path Angle versus Time, Gains selected to match SP2 (see corresponding Figures 31 and 32) 


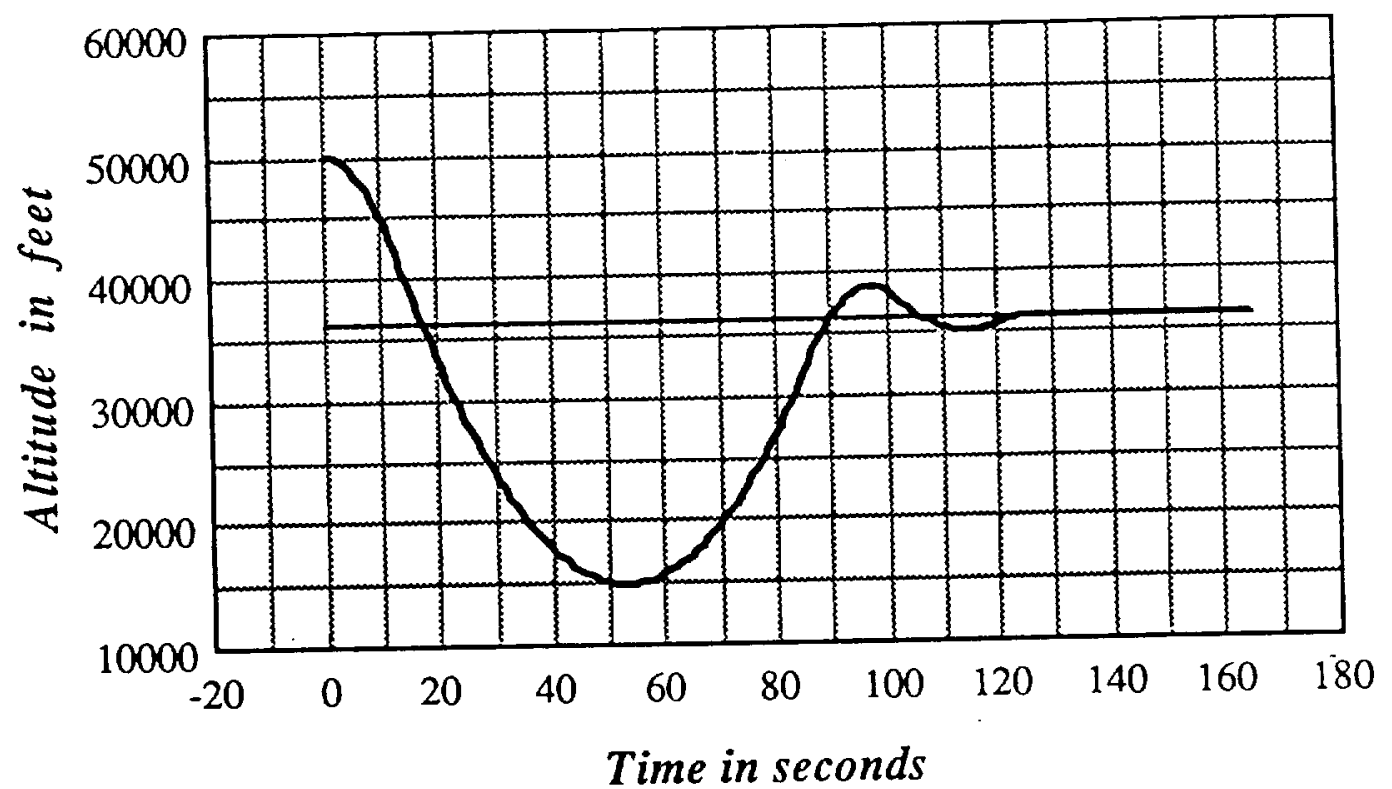

Figure 41. NLT Tracking Constant Energy Reduced Solution, Altitude versus Time, $\mathrm{L} / \mathrm{W} \leq 2.0$, Gains selected to match SP2 (see corresponding Figures 33 and 34 )

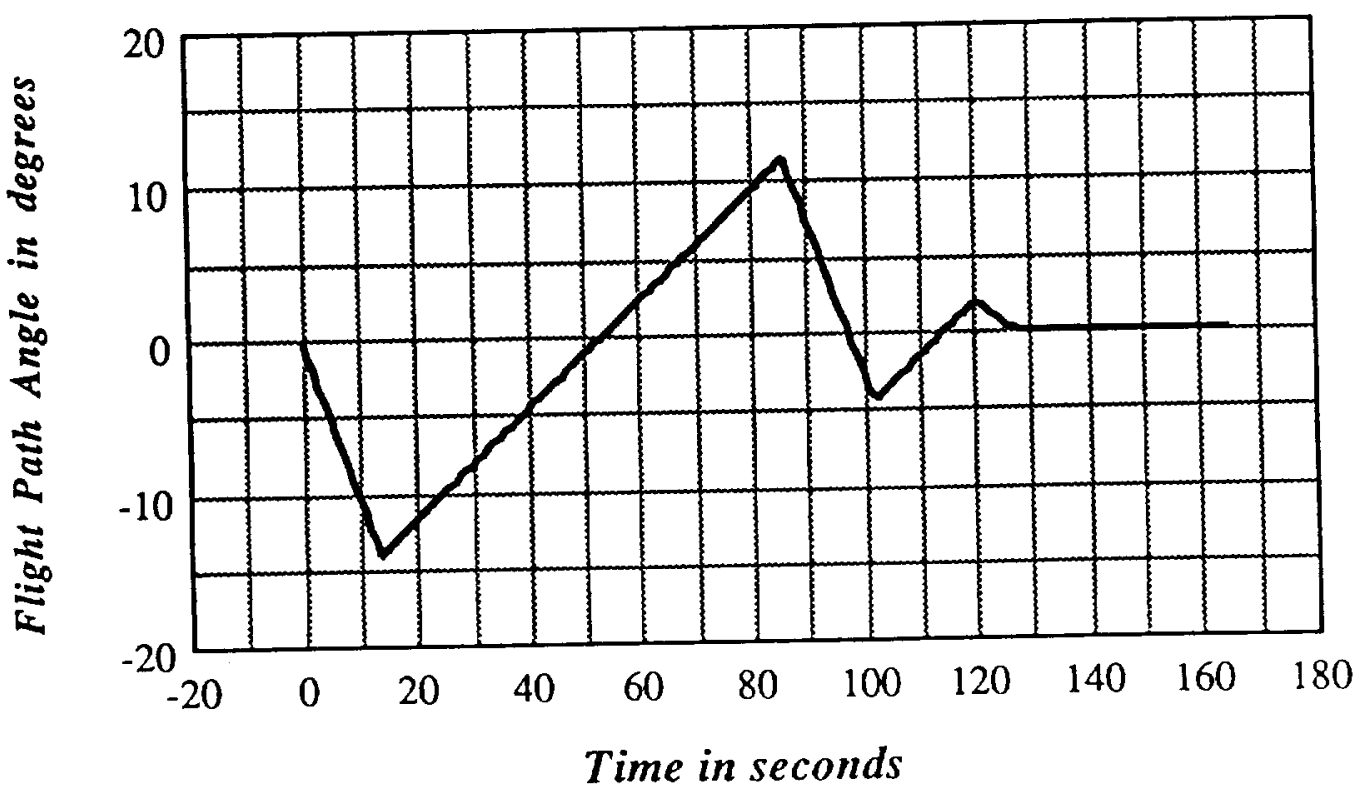

Figure 42. NLT Tracking Constant Energy Reduced Solution, Flight Path Angle versus Time, $\mathrm{LW} \leq 2.0$, Gains selected to match SP2 (see corresponding Figures 33 and 34 ) 


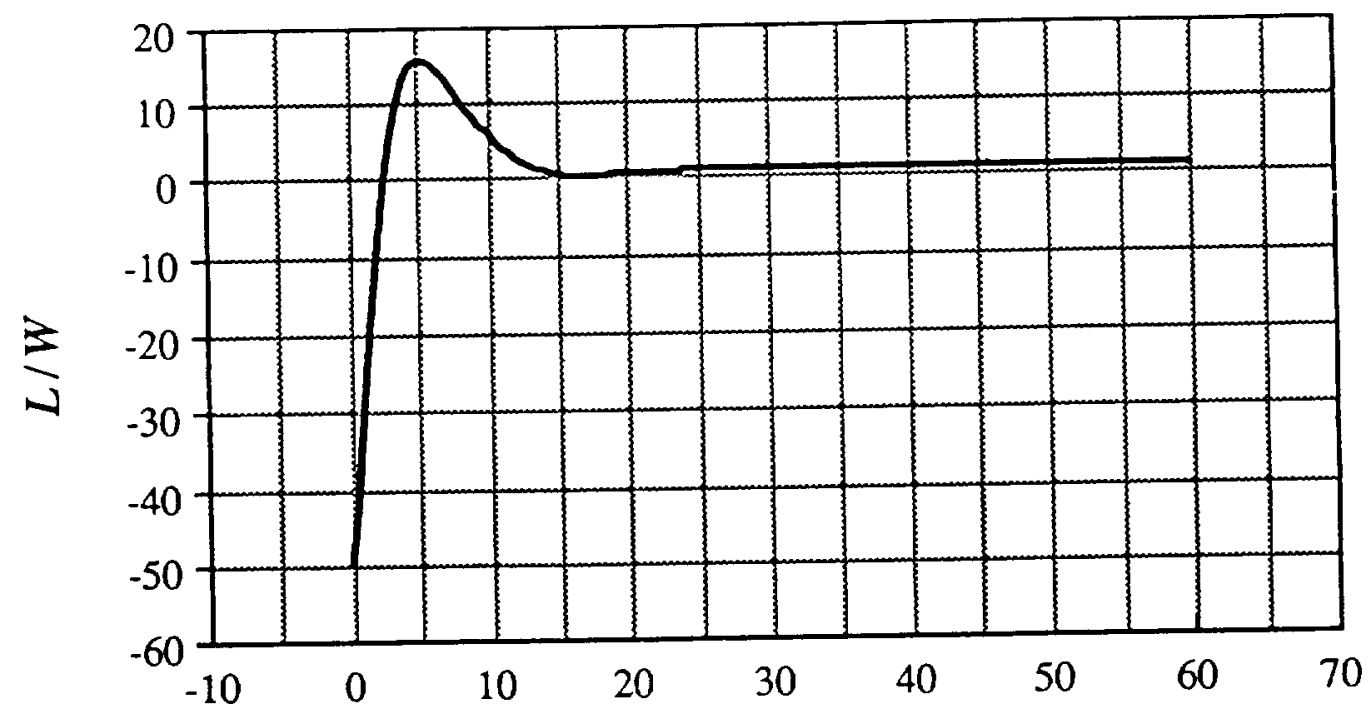

Time in seconds

Figure 43. L/W for NLT Controller Tracking Constant Energy Reduced Solution, Gains selected to match SP2 (see corresponding Figures 39 and 40)

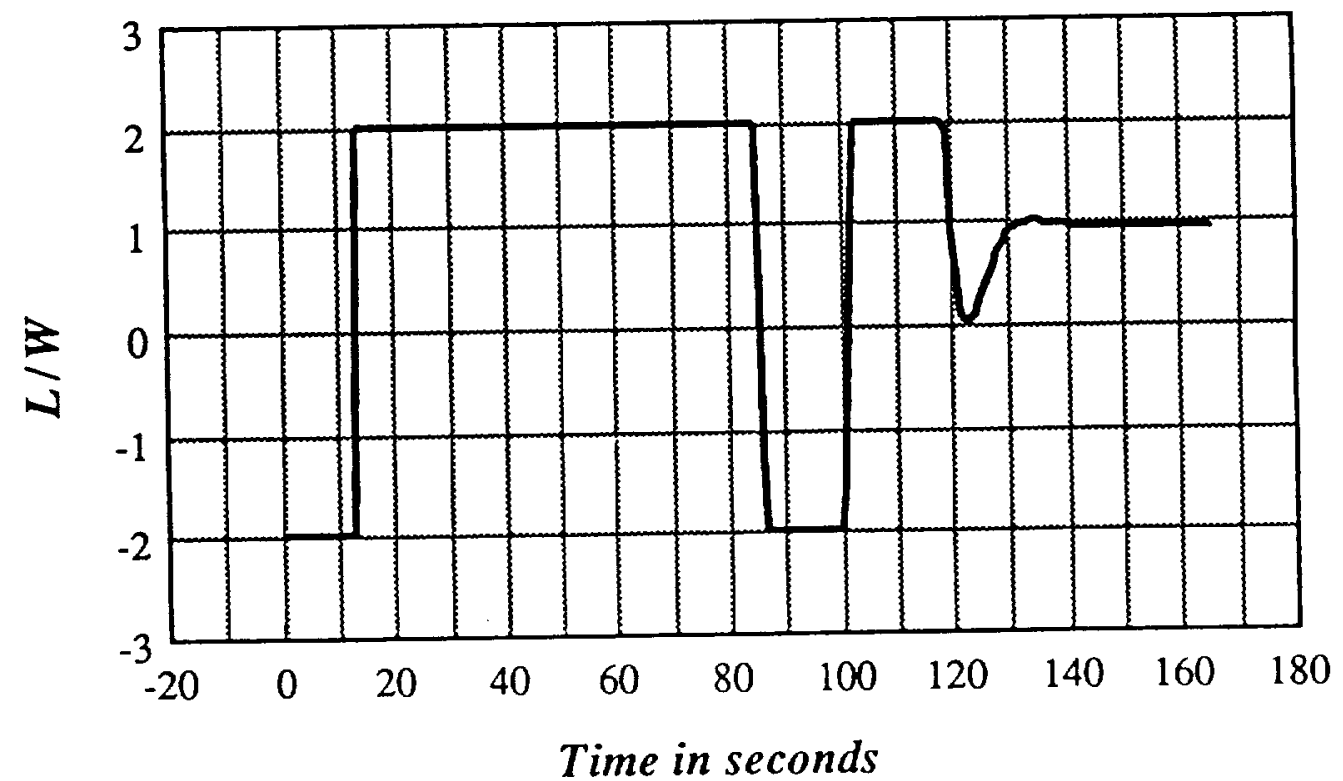

Figure 44. NLT Controller Tracking Constant Energy Reduced Solution, $L / W \leq 2.0$, Gains selected to match SP2 (see corresponding Figures 41 and 42) 


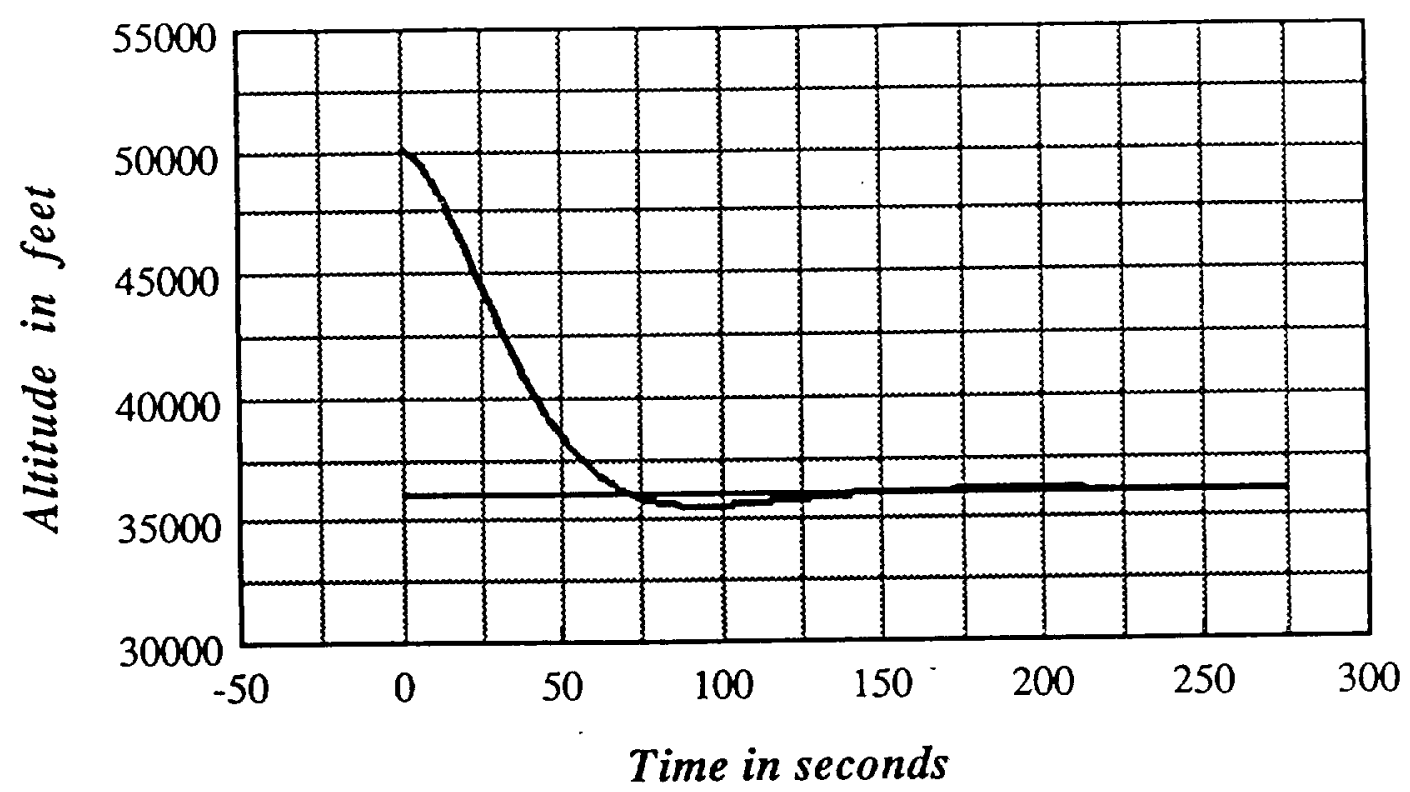

Figure 45. NLT Controller Tracking Constant Energy Reduced Solution, Altitude versus Time, Gains picked to give a damping ratio of 0.7 and a $2 \%$ settling time of 120.0 seconds

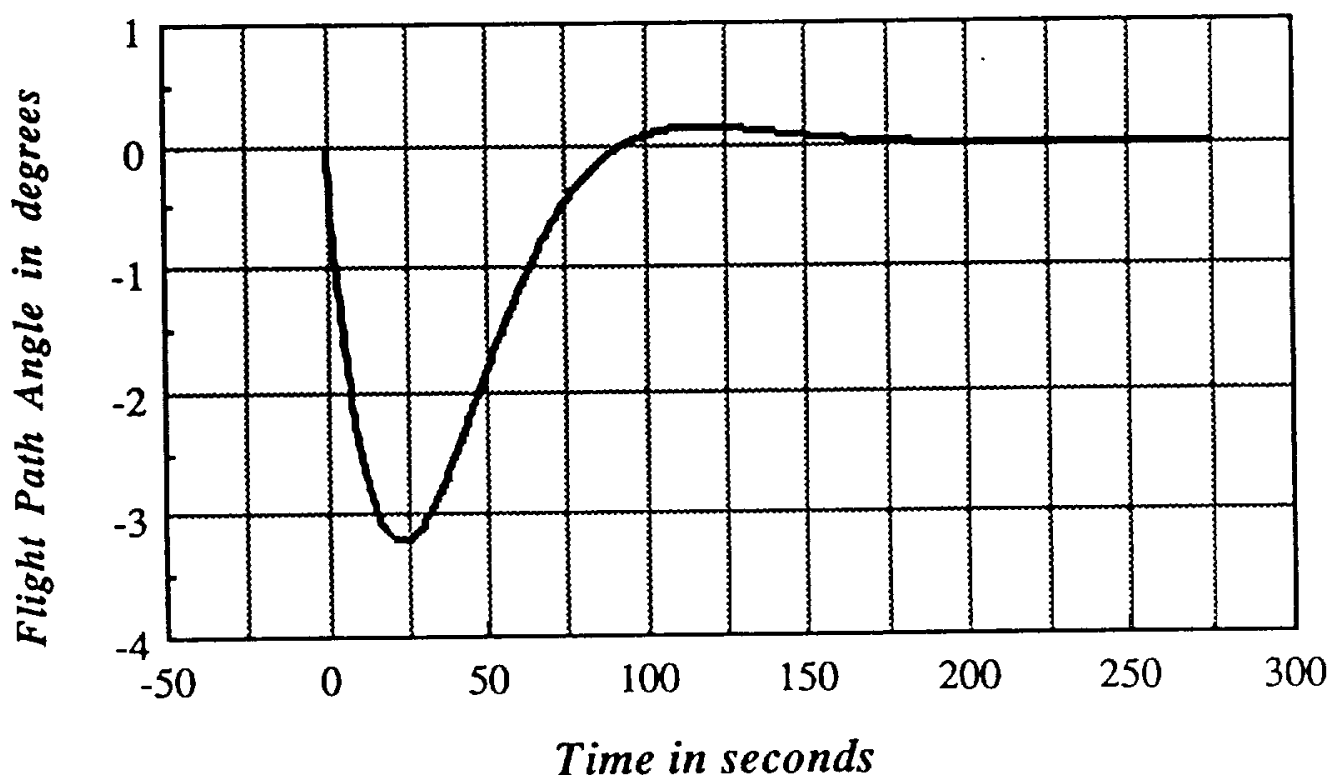

Figure 46. NLT Controller Tracking Constant Energy Reduced Solution, Flight Path Angle versus Time, Gains picked to give a damping ratio of 0.7 and a $2 \%$ settling time of 120.0 seconds 


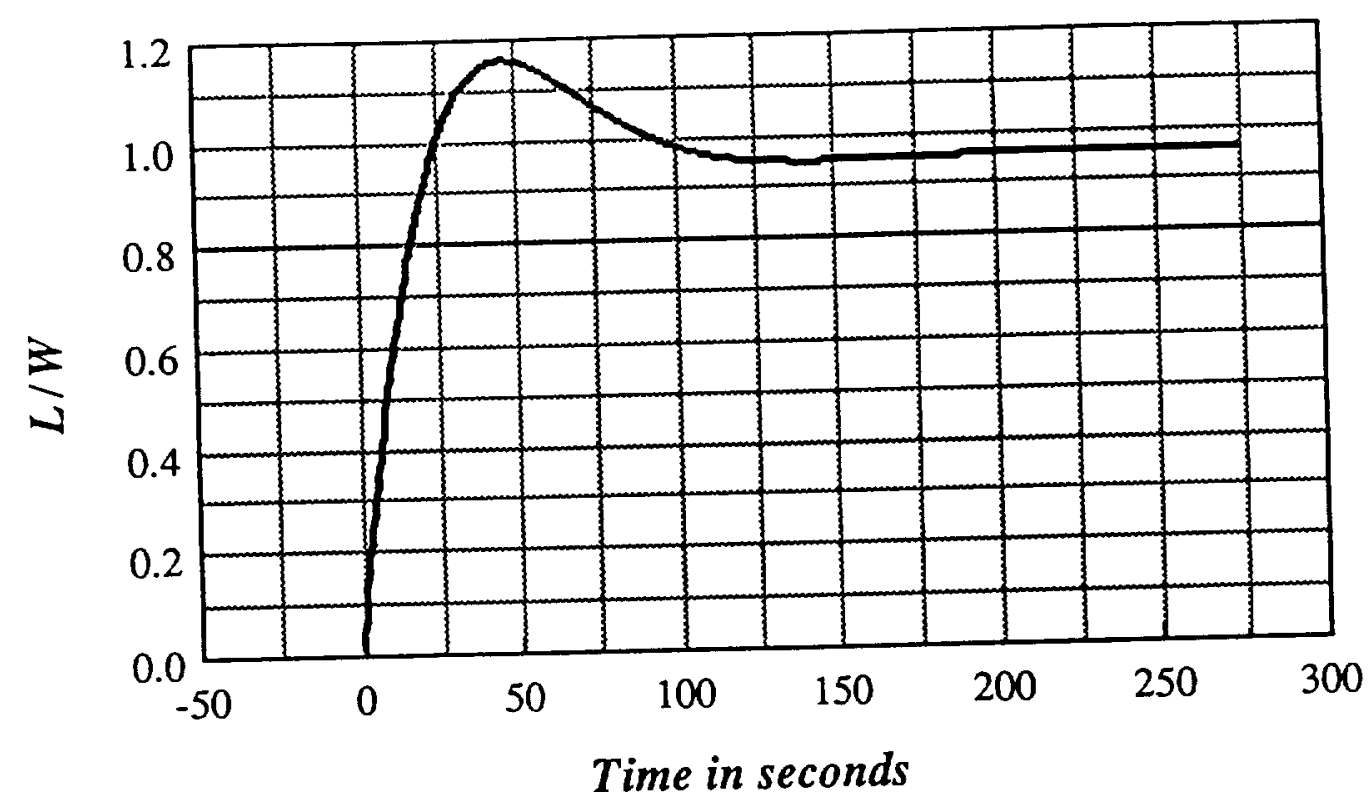

Figure 47. LNW for NLT Controller Tracking Constant Energy Reduced Solution, Gains picked to give a damping ratio of 0.7 and a $2 \%$ settling time of 120.0 seconds (see corresponding Figures 45 and 46)

\section{Inclusion of a Maximum Dynamic Pressure Constraint}

Recall the definition given for dynamic pressure, $q$, and velocity, $\mathrm{V}$ :

$$
q=1 / 2 \rho V^{2}=q\left(E_{o}, r\right) \text { where } V=\left[2\left(E_{0}+\mu / r\right)\right]^{1 / 2}
$$

It is desired to constrain $q$ in the boundary layer so that $q \leq q_{\max }$. Note that in the boundary layer, $\mathrm{E}$ is held constant at its reduced solution value and $\mathrm{r}$ resumes it status as a state variable. Thus, in contrast to the reduced solution in which the dynamic pressure constraint was a function of state and control, in the boundary layer we must enforce a pure state constraint. The associated tangency conditions which must be met at a juncture between unconstrained and state constrained arcs, along with possible discontinuitites in the costates, the Hamiltonian, and the control $[52,69,70]$, appear to make a straight 
forward application of the available theory difficult at best. A means for handling a pure state constraint in the boundary layer is currently under investigation.

The lift control solution derived via the nonlinear transformation technique discussed when considering the unconstrained case applies equally well to the dynamic pressure constrained case. Figures 48 and 49 present the altitude and flight path angle time histories generated using the NLT controller to track constant conditions associated with the dynamic pressure constrained case for several values of $\mathrm{K}_{\mathrm{p}}$ and $\mathrm{K}_{\mathrm{d}}$, as given in Table 2. The corresponding lift control histories are presented in Figure 50. As indicated in Figure 48, the damping ratio can be chosen to avoid violation of the constraint boundary. In the next section the NLT control law is used to numerically simulate ascent to orbit along the $q$ constrained reduced solution fuel optimal climb path.

Table 2 NLT Gain Variations Depicted in Figures 48-50

\begin{tabular}{lllll}
\hline$\zeta$ & $\omega_{\mathrm{n}}(\mathrm{rad} / \mathrm{sec})$ & $\mathrm{T}_{\mathrm{s}}(\mathrm{sec})^{*}$ & $\mathrm{~K}_{\mathrm{p}}$ & $\mathrm{K}_{\mathrm{d}}$ \\
\hline 0.707 & 0.047 & 120.0 & 0.0022 & 0.0665 \\
0.854 & 0.039 & 120.0 & 0.0015 & 0.0666 \\
1.0 & 0.033 & 120.0 & 0.0011 & 0.0666
\end{tabular}

* $2 \%$ Settling Time given by $4 / \zeta \omega_{n}$ 


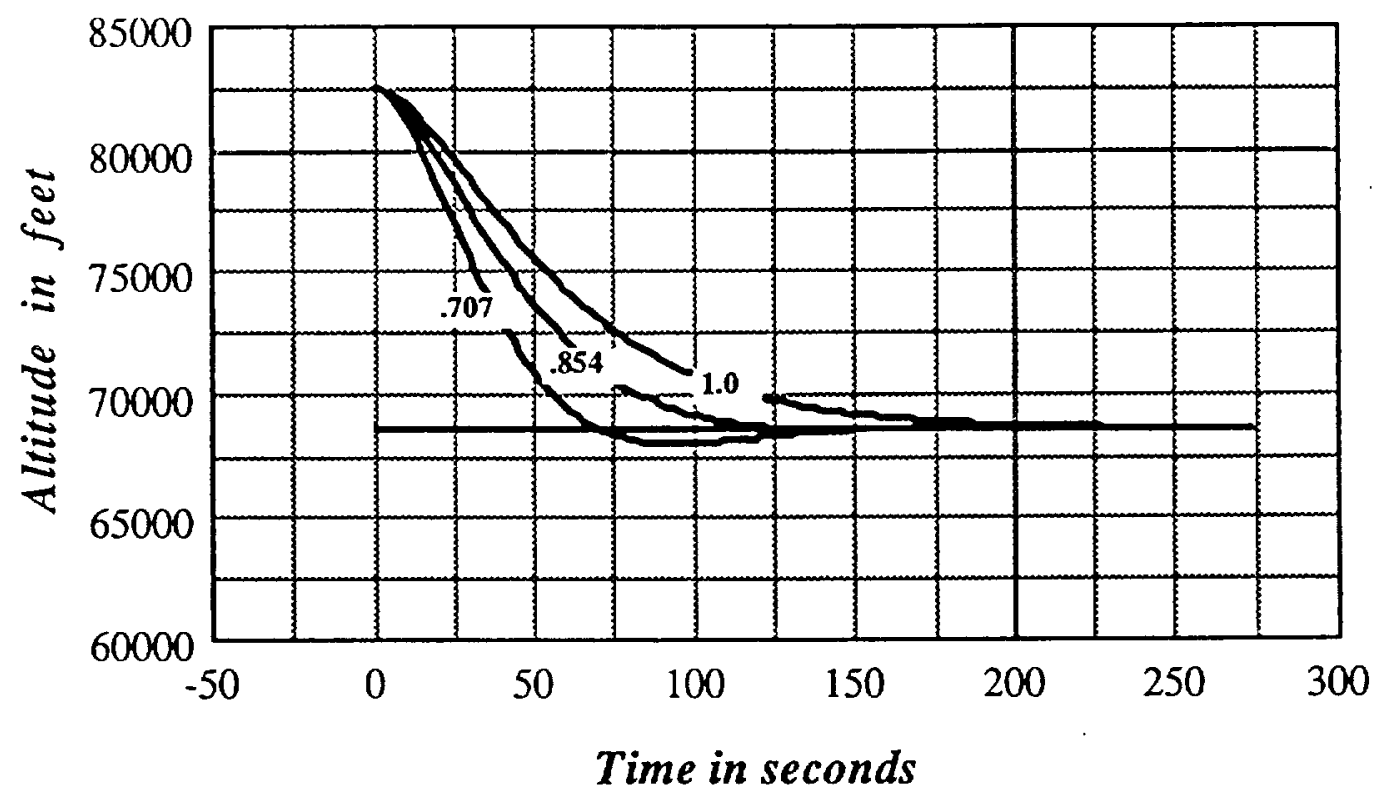

Figure 48. NLT Controller Tracking Constant Energy q Constrained Reduced Solution, Altitude versus Time, Gains picked to yield various damping ratios (see Table 2 ) but to maintain a $2 \%$ settling time of 120.0 seconds

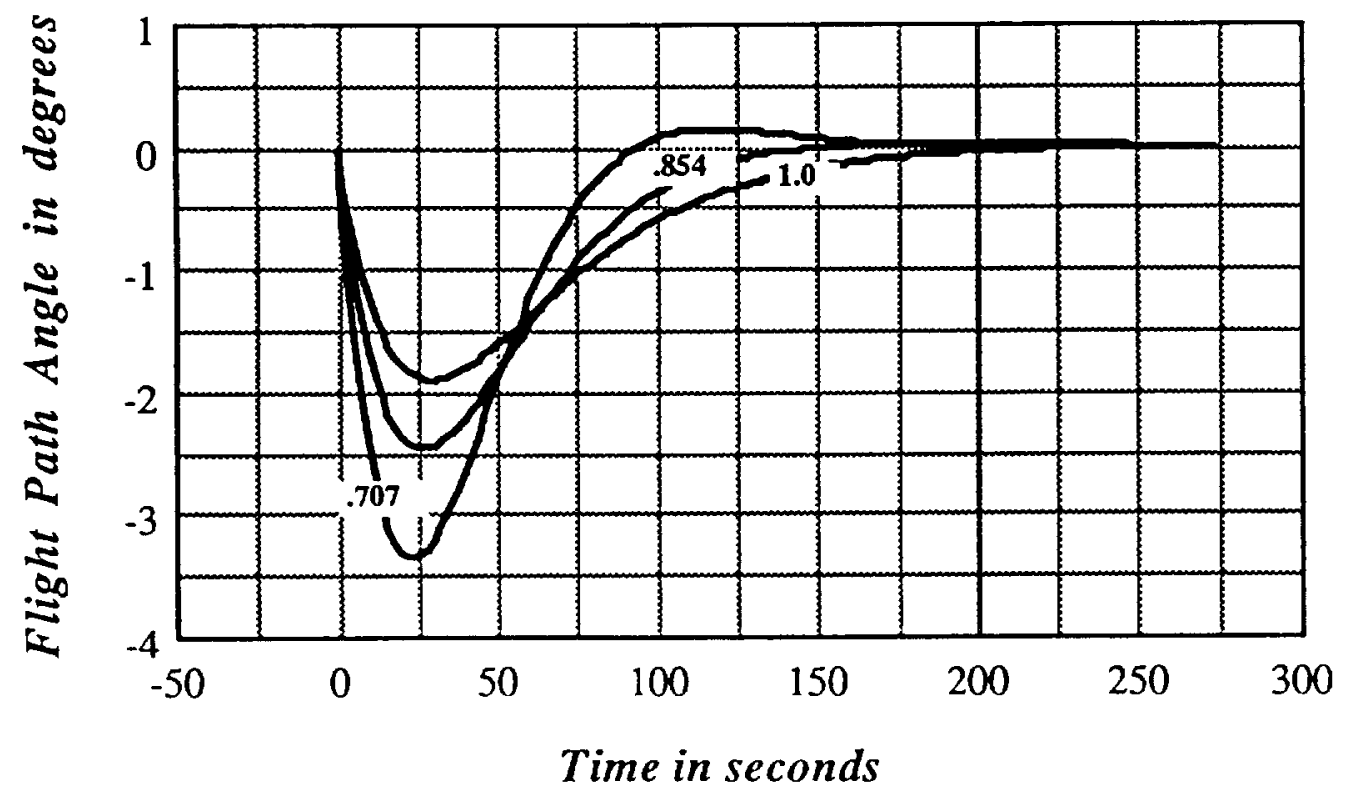

Figure 49. NLT Controller Tracking q Constrained Constant Energy Reduced Solution, Flight Path Angle versus Time, Gains selected to yield various damping ratios (see Table 2 ) but to maintain a $2 \%$ settling time of 120.0 seconds 


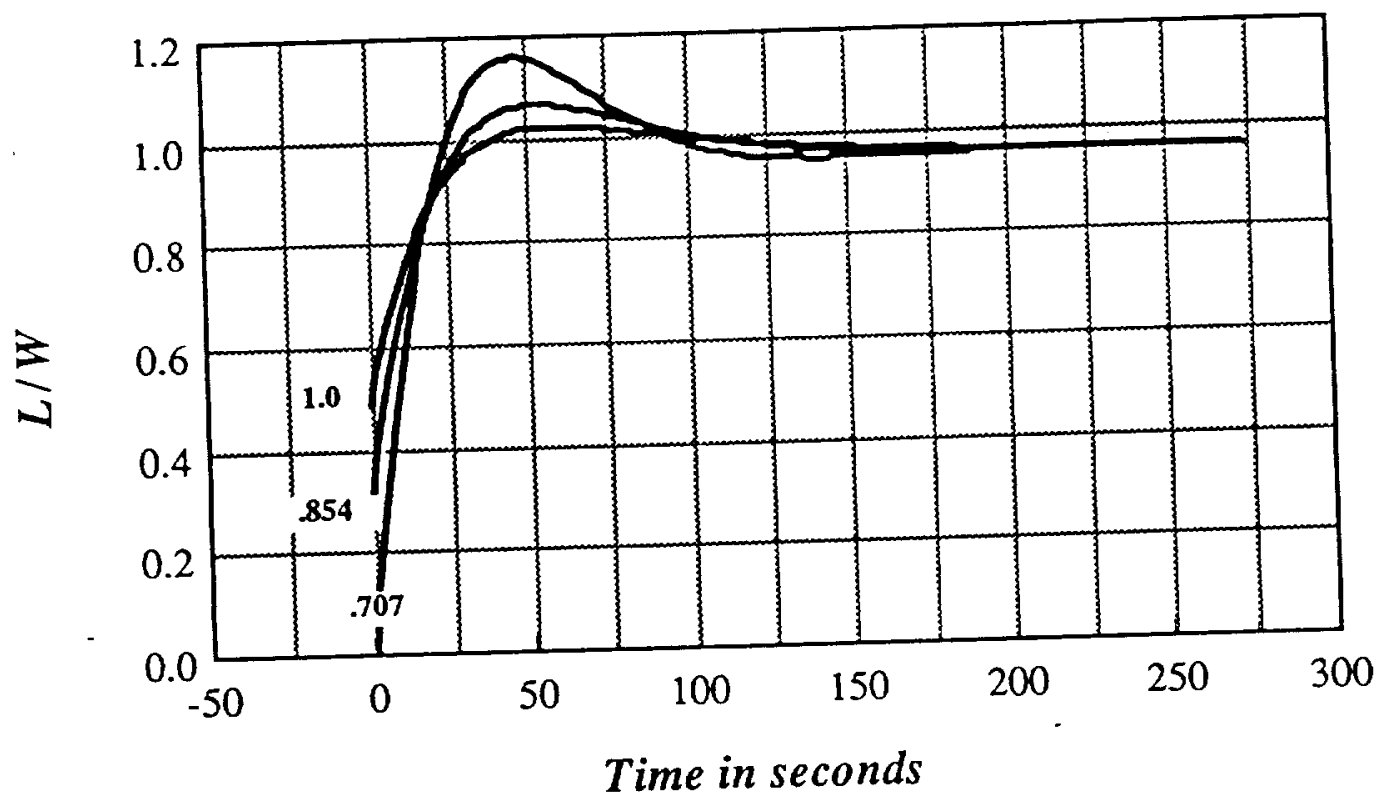

Figure 50. L/W for NLT Controller Tracking Constant Energy Reduced Solution, Gains selected to yield various damping ratios (see Table 2) but to maintain a $2 \%$ settling time of 120.0 seconds (see corresponding Figures 48 and 49) 


\section{Numerical Simulation Results}

A numerical simulation of the dynamic pressure constrained ( $q \leq 2000 \mathrm{psf}$ ) fuel-optimal energy climb was carried out using the feedback law (NLT) given in equation (110) with gains selected to yield a damping ratio of 7 and a $2 \%$ settling time of 120.0 seconds. For the purpose of comparison, the value of the first time derivative of $r_{0}$ was initially taken to be zero all along the path. An altitude perturbation of 14,000 feet was specified at the initial energy level and the initial flight path angle was specified as zero. The resulting trajectory, superimposed over the $q$ constrained reduced solution, is presented along with the associated lift control time history in Figure 51. As expected, a slight lag is evident throughout the trajectory and a large value of lift is commanded upon reaching the altitude discontinuity. Figure 52 presents a second numerical simulation given the same initial conditions and controller gains but with the first time derivative of $r_{0}$ computed using a forwards difference in energy equal to 1/50th the final energy level. Excellent tracking of the reduced solution path is now achieved with the vehicle flying slightly above the dynamic pressure constraint boundary. Note that in either case the addition of rocket propulsion is deemed advantageous via (52) and switched on. The flight path angles required to fly either of the trajcetories shown in Figures 51 and 52 are presented as Figures 54 and 55, respectively. In either case it remains less than four degrees. Thus the assumption of zero flight path angle in the reduced solution turns out to be very good. Approximately sixty percent of the total vehicle mass is consumed in climbing to the final energy given an initial weight of 200,000 pounds. This percentage of gross weight consumed is equivalent to that estimated as required to follow the reduced solution (see discussion on page 55) and it thus appears that further optimization of the altitude and flight path angle dynamics is of no value in minimizing the performance index. The longitudinal acceleration is estimated as $0.6 \mathrm{~g}$ 's when averaged over the entire trajectory but is about 2.0 g's through the Mach range $M=9$ to 13 where the excess thrust available is greatest.

There is still a lag in the response at the energy level where the reduced solution altitude is discontinuous. This lag can be avoided by calculating $r_{0}$ and the desired rate of climb using a "forward look" procedure. This simply consists of performing the calculations of $r_{0}$ 
and $d r_{o} / d t$ at an energy level slightly higher (ahead of) the current energy level. It should be possible to derive some intelligent logic that adjusts the controller gains and the magnitude of the "forward look" as a function of the current vehicle state. An investigation of this matter is proposed as a future research objective in the next section. 


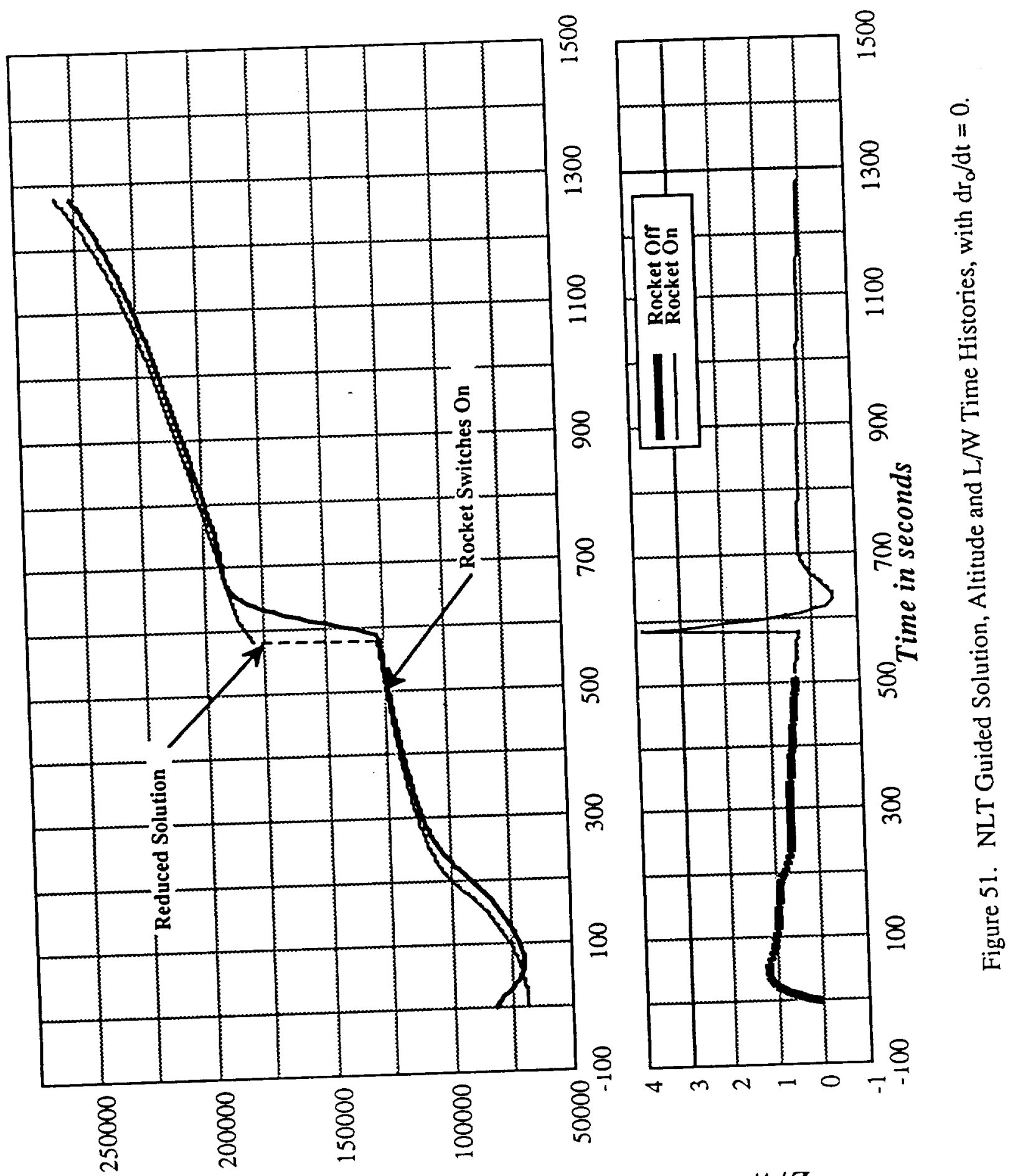

1วәf $u ?$ opn!!nV

$M / T$ 


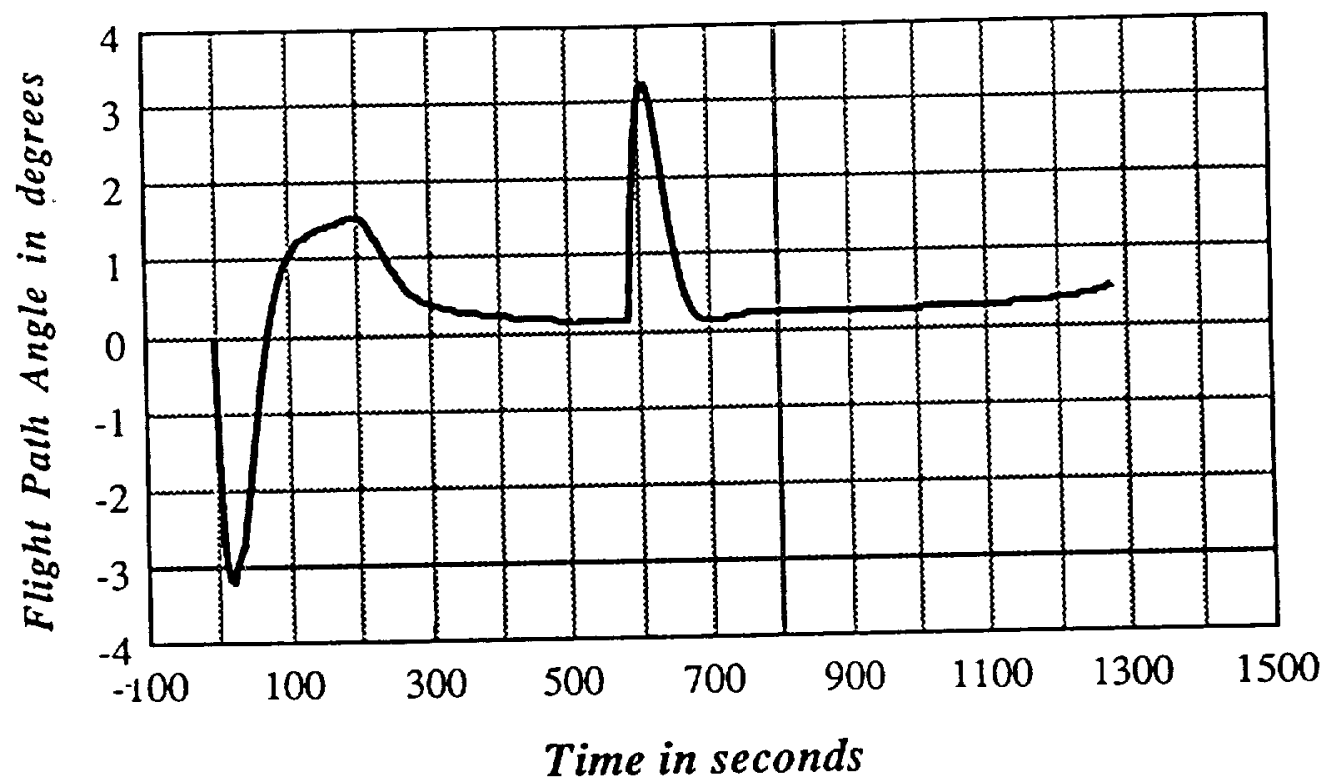

Figure 53. NLT Guided Solution, Flight Path Angle Time History, No estimate of desired rate of climb.

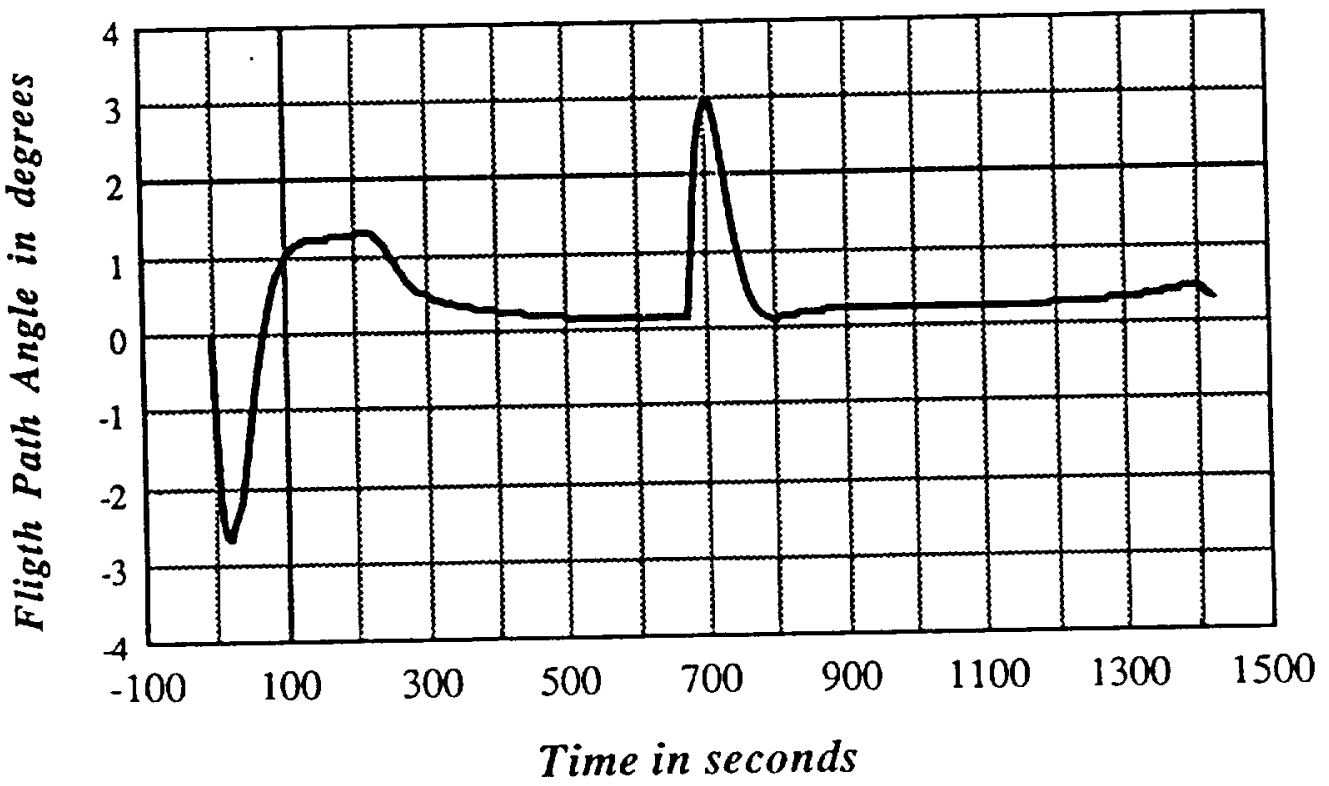

Figure 54. NLT Guided Solution, Flight Path Angle Time History, with estimate of desired rate of climb. 


\section{Conclusions and Topics for Future Research}

This report documents the work accomplished during the period July 1987 to September 1988. All of the work documented in the progress report previously submitted for the period July 1987 to December 1987 is included in this report with several very important corrections implemented within the SCRAMJET performance code. These corrections removed several disparities reported between our results and those of cited references as follows:

- The SCRAMJET model, based in large part on empirical relations, now exhibits qualitative behavior similar to that reported by other sources.

- Sizing of the engine inlet area by estimating the vehicle's level flight envelope now indicates an increased requirement for inlet area as Mach number increases. This is the expected trend that was not observed previously due to incorrect SCRAMJET modeling.

It was reported previously that the analytic aerodynamic vehicle model being developed was not yet a satisfactory tool for use in trajectory optimization and guidance law development. In particular it was stated that the hypersonic aerodynamic model satisfactorily predicts drag variation with lift and is responsive to control surface deflections but poorly estimates vehicle lift and its variation with angle of attack. The work completed in this area as of December 1987 is documented in Appendix A of this report. As cited in the appendix, suitable corrections to the method may take the form of additional geometric complexity but such have not been implemented at this time.

A number of important modeling issues have not yet been addressed and many of these may well have a sizable influence on vehicle design, vehicle performance, and the nature of optimal trajectories. These include sensitivity of SCRAMJET performance to angle of attack variation, the incorporation of means for accounting for fuel pre-heat due to its 
circulation as a coolant prior to combustion, and the addition of fuel into the combustor in excess of the stoichiometric ratio for the purpose of engine structural cooling. Another important effect not yet accounted for is the possibility of a large component of SCRAMJET thrust normal to the body longitudinal axis. This force, which contributes to overall vehicle lift and which can cause a large nose down pitching moment, may have a significant impact on vehicle performance and should be properly modeled. The vehicle model's aerodynamic envelope must also be enlarged to incorporate subsonic and supersonic flight and alternate modes of propulsion for these lower speeds. Additional controls such as variable geometry inlets may also need to be modeled in the future.

It is not yet clear whether or not intermediate values of SCRAMJET thrust are desirable during ascent or an abort maneuver. Nor is it clear how a throttle back would be achieved. Most likely one or more engines modules would be shut down and their intakes closed to reduce drag. This leads to an optimal control problem in which the control variable is constrained to take on only a finite number of discrete values. The minimum principle still holds in such a case but the necessary conditions used to formulate the control solution presented in this report no longer apply. New necessary conditions would have to be derived to proceed with the analysis.

Several trajectory constraints have been ignored in the analysis thus far. These include acceleration limits, angle of attack limits, and most importantly, aerodynamic heating constraints. Of note is the fact that the incorporation of these constraints will likely remove the large altitude discontinuity observed in the reduced solution in the unconstrained and dynamic pressure constrained cases.

Consideration of either dynamic pressure or aerodynamic heating constraints leads to a state constrained boundary layer problem. Great difficulty has been encountered in attempting to construct a linearized boundary layer solution when a state constraint is to be enforced since no theory has been developed for such a case. The theoretical aspects of this issue need to be addressed and, if possible, a suitable methodology for handling such problems should be developed. In addition, alternate approaches, such as the forced separation of altitude and flight path angle dynamics, need to be investigated and compared with the linearized approach. The suboptimal nonlinear transformation approach outlined in Section 5.2.2 shows great promise with regard to overcoming some of the shorcomings of 
the linearized approach. An extension of this technique to include transformation of the performance index so that a linear quadratic cost function appears in the linear space would result in a very powerful optimal approach and should also be investigated.

Proposed future research tasks are detailed below.

- Further develop the vehicle model as follows:

- Extend the aerodynamic model to the subsonic and supersonic regimes and define a suitable means for transitioning between each flight regime's data set.

- Develop suitable turbojet and ramjet engine models.

- Investigate additional SCRAMJET modeling issues including variation of engine performance with angle of attack, additional thrust derived from fuel preheat, additional fuel flow required for engine structural cooling at the higher Mach numbers, a possible requirement for variable geometry, and dual-mode (ramjet-SCRAMJET) operation.

- Incorporate the Generic Hypersonic Aerodynamic Model Example (GHAME) as an alternate aerodynamic model.

- Develop suitable analytic (to the extent possible) aerodynamic models that will allow trajectory studies to be conducted on configurations for which windtunnel data is not available.

- Extend the analysis to include:

- Fight in the Mach range 0-5 (This task will require consideration of optimal propulsion system transitions in the subsonic and supersonic flight regimes.) 
- The component of thrust normal to the flight path

- Variable SCRAMJET thrust (This task may require the derivation of necessary conditions associated with discrete variation of SCRAMJET thrust since it is likely that SCRAMJET thrust variation will be achieved by turning on or off individual engine modules.)

- Aerodynamic heating, acceleration, angle of attack, and lift constraints

- Orbital insertion end conditions

- Three dimensional dynamics as may be required for lift modulation, orbital plane change, and abort maneuvers

- A rotating oblate Earth

- A nonstationary atmosphere

- Continue the derivation of a suitable guidance algorithm.

- Address the theoretical issues associated with the inclusion of a state variable constraint in the boundary layer analysis. If possible devise a method for synthesizing a linearized boundary layer lift control solution for the constrained case.

- Consider the forced separation of altitude and flight path angle dynamics as an alternate approach to handling the boundary layer problem.

- Consider the derivation of intelligent rules for selecting gains and estimating the desired rate of climb in the NLT lift control solution. 
- Consider the possibility of an exact nonlinear transformation of the boundary layer necessary conditions to a linear optimal control problem with a quadratic index of performance.

- Extend the Singular Perturbation formulation to include the out-of-verticalplane dynamics as an additional layer.

- Evaluate the resulting real-time guidance algorithms in nonreal-time simulation studies.

- Compare the guided solutions with exact numerical solutions obtained using a multiple shooting algorithm.

- Examine the robustness of the guided solutions to variations in atmospheric conditions, off-nominal engine performance, and other modeling uncertainties.

- Conduct sensitivity studies to examine the impact of vehicle sizing parameters on the nature of the optimal trajectories. 


\section{References}

[1] Pioneering the Space Frontier, The Report of the National Commission on Space,Bantam Books, Inc., New York, NY, 1986.

[2] Ride, S. K., Leadership and America's Future in Space, Aviation Week and Space Technology, Peoria, IL, 1987.

[3] Davis, J. G., Jr., and S. C. Dixon, "Beyond Simulation," Aerospace America, July 1988, pp. 38-42.

[4] National Aero-Space Plane, A Technology Development and Demonstration Program to Build the X-30, United States General Accounting Office Report to Congressional Committees, GAO/NSIAD-88-122, April 1988.

[5] Rosen, C. C., III, Burger, R. J., and Sigalla, A., "Aeronautical Technology 2000: A Projection of Advanced Vehicle Concepts," presented at the 1984 AIAA/AHS/ASEE Aircraft Design Systems and Operations Meeting, AIAA Paper \# 84-2501.

[6] Hearth, D. P., and Preyss, A. E., "Hypersonic Technology - Approach to an Expanded Program," Astronautics \& Aeronautics, December, 1976.

[7] Calise, A. J., Flandro, G. A., and Corban, J. E., "Trajectory Optimization and Guidance Law Development for NASP Applications," A proposal submitted to NASA LaRC by the Georgia Institute of Technology, School of Aerospace Engineering, April, 1987.

[8] Williams, R. M., "National Aero-space Plane:Technology for America's Future," Aerospace America, November 1986, p 18.

Bradt, J. E., Hardtla, J. W., and Cramer, E. J., "An Adaptive Guidance Algorithm for Aerospace Vehicles, Proceedings of the 1985 AIAA Guidance, Navigation, and Control Conference, Paper \# 85-1917, pp.415-423.

[10] Hardtla, J. W., Piehler, M. J., and Bradt, J. E.,"Guidance Requirements for Future Launch Vehicles," Proceedings of the 1987 AIAA Guidance, Navigation, and Control Conference, Paper \#87-2462, pp. 988-993.

[11] Bryson, A. E., Desai, M. N., and Hoffman, W. C., "Energy-State Approximation in Performance Optimization of Supersonic Aircraft," Journal of Aircraft, Vol. 6, No. 6, Nov.-Dec. 1969 , pp. 481-488.

[12] Calise, A. J., "Singular Perturbation Methods for Variational Problems in Aircraft Fight, IEEE Transactions on Automatic Control, Vol. AC-21, No. 3, June 1976, pp. 345-353. 
[13] Calise, A. J., "Extended Energy Management Methods for Flight Performance Optimization," AIAA Journal, Vol. 15, No. 3, March 1977, pp. 314-321. [14] Calise, A. J., "Singular Perturbation Techniques for On-Line Optimal Flight-Path
Control," AIAA Journal of Guidance \& Control, Vol. 4, No. 4, 1981, pp. 398-405.

[15] Heppenheimer, T. A., "Launching the Aerospace Plane," High Technology, July,

[16] Creel, T. R., Jr. and Penland, J. A., "Low-Speed Aerodynamic Characteristics of a Hypersonic Research Airplane Concept Having a $70^{\circ}$ Swept Delta Wing," NASA TM
$X-71974,1974$.

[17] Penland, J. A., Fournier, R. H., and Marcum, D. C., Jr., " Aerodynamic Doubteristics of a Hypersonic Research Airplane Concept Having a $70^{\circ}$ Swept

[18] Clark, L. E., and. Richie, C. B., " Aerodynamic Characteristics at Mach 6 of a Xypersonic Research Airplane Concept Having a $70^{\circ}$ Swept Delta Wing," NASA TM
S 1977.

[19] Penland, J. A., Hallissy, J. B., and Dillion, J. L., " Aerodynamic Characteristics of Mach Numbers from Airplane Concept Having a $70^{\circ}$ Swept Double-Delta Wing at TP-1552, 1979.

[20] Weidner, J. P., Small, W. J., and Penland, J. A., "Scramjet Integration on Hypersonic Research Airplane Concepts," Journal of Aircraft, Vol. 14, No. 5, pp.
460-466, May, 1977.

[21] "X-30 Technology Advancing Despite Management Rift," Aviation Week \& Space
Technology, March 7, 1988, p.36. [22] Covault, C., "X-30 Research Narrowing Hypersonic Design Options," Aviation
Week and Space Technology, pp. 32-33, April 27, 1987.

[23] Anderson, J. D., Jr., "A Survey of Modern Research in Hypersonic Aerodynamics," Proceedings of the ALAA $17^{\text {th }}$ Fluid Dynamics, Plasma Dynamics, and Lasers Conference, June 25-27, 1984, Snowmass, Colorado, AIAA-84-1578.

[24] Kerrebrock, J. L., Aircraft Engines and Gas Turbines, MIT Press, Cambridge,
Massachusetts, 1977. [25] Billig, F. S., "Design Considerations of Supersonic Combustion Ramjets," ALAA-
86-0159, ALAA 24th Aerospace Science Meeting, Jan 6-9, 1986.

[26] Northam, G. B. and G. Y. Anderson, "Supersonic Combustion Ramjet Research at Langley," AIAA-86-0159, AIAA 24th Aerospace Science Meeting, Jan 6-9, 1986.

[27] Avery, W. H. and Dugger, G. L., "Hypersonic Airbreathing Propulsion," 
Astronautics and Space Engineering, June 1964, pp 42-47.

[28] Waltrup, P. J., "Liquid Fueled Supersonic Combustion Ramjets: A Research Prospective of the Past, Present, and Future," AIAA-86-0158, AIAA 24th Aerospace Sciences Meeting, Jan. 6-9, 1986.

[29] Merrifield, J. T., "Aerojet TechSystems Develops Hypersonic Airctaft Engine," Aviation Week \& Space Technology, Oct. 14, 1985, pp. 57-61.

[30] Guy, R. W. et. al., "Operating Characteristics of the Langley Mach 7 Scramjet Facility," NASA TM 81929, March, 1981.

[31] Martin, J. A., "An Evaluation of Composite Propulsion for Single-Stage-to-Orbit Vehicles Designed for Horizontal Take-off," NASA TM X-3554, November, 1977.

[32] White, M. E., Drummond, J. P., and Kumar, A. J., "Evolution and Status of CFD Techniques for Scramjet Applications," ALAA-86-0160, AIAA 24th Aerospace Sciences Meeting, January 6-9, 1986.

[33] Small, W. J., Weidner, J. P., and Johnston, P. J., "Scramjet Nozzel Design and Analysis as Applied to a Highly Integrated Hypersonic Research Airplane," NASA TN D-8334, 1976.

[34] Zucrow, M. J., Aircraft and Missle Propulsion, John Wiley \& Sons, 1958.

[35] Hill, P. G. and Peterson, C. R., Mechanics and Thermodynaimcs of Propulsion, Addison-Wesley Publishing Company, 1965.

[36] Jane's All the World's Aircraft, edited by J. W. R. Taylor, "Pratt \& Witney RL10 Rocket Engine," p. 764, Franklin Watts, Inc., New York, NY, 1976.

[37] Brauer, G. L., Cornick, A. R., Habeger, F. M., and R. Stevenson, "Program to Optimize Simulated Trajectories (POST), Volume 1: Formulation Manual, NASA CR-132689, 1975.

[38] "X-30 Research Narrowing Hypersonic Design Options," Aviation Week \& Space Technology, April 27, 1987, p. 32.

[39] Johnston, P. J., Whitehead, A. H., Jr., and Chapman, G. T., "Fitting Aerodynamics and Propulsion into the Puzzle," Aerospace America, September, 1987.

[40] Calise, A.J., Moerder, D.D., "Singular Perturbation Techniques for Real Time Aircraft Trajectory Optimization and Control," NASA Contractor Report 3597, August, 1982.

[41] Calise, A.J., "Extended Energy Management Methods for Flight Performance Optimization", AIAA J., Vol. 15, No. 3, 1977.

[42] Price, D., Calise, A.J., Moerder, D., "Piloted Simulation of an On-Board Trajectory Optimization Algorithm," AIAA J. of Guid. and Cont., Vol. 7, No. 3, May, 1984. 
[43] Jones, F.P., Duke, E.L., Calise, A.J., "Flight Test Experience from a ThreeDimensional Optimal Intercept of a Maneuvering Target," 2nd International Symposium on Differential Games, Williamsburg, VA, Aug., 1986.

[44] Calise, A.J., Bae, G., "Optimal Heading Change with Minimum Energy Loss for a Hypersonic Gliding Vehicle," to be presented at the AIAA Atmospheric Flight Mechanics Conf., Aug., 1987.

[45] Marec, J. P., Optimal Space Trajectories, Elsevier Scientific, New York, NY, 1979.

[46] Vinh, N. X., Optimal Trajectories in Atmospheric Flight, Elsevier Scientific, New York, NY, 1981.

[47] Etkin, B., Dynamics of Atmospheric Flight, John Wiley \& Sons, New York, NY, 1981.

[48] Schoettle, U. M. "Performance Analysis of Rocket-Ramjet Propelled SSTO Vehicles," $36^{\text {th }}$ Congress of the International Astronautical Federation, Stockholm, Sweden, October 7-12, 1985, IAF-85-133.

[49] Tauber, M. E., Menees, G. P., and Adelman, H. G., "Aerothermodynamics of Transatmospheric Vehicles," ALAA/ASME 4th Joint Thermophysics and Heat Transfer Conference, June 2-4, 1986, Boston, MA, AlAA Paper \# ALAA-86-1257.

[50] Taylor, L. W., Gracey, C., and Armstrong, C. D., "A Guidance-Motivated Sensitivity Analysis of an Aero-Assisted Boost Vehicle," presented at the 1986 AIAA Conference on Navigation, Guidance and Control, No. 86-2103, pp. 420428.

[51] Kelly, H. J., Cliff, E. M., and Weston, A. R., "Energy State Revisited," Optimal Control Applications \& Methods, Vol. 7, pp. 195-200, 1986.

[52] Bryson, A. E., Jr., and Ho, Yu-Chi, Applied Optimal Control, Hemisphere Publishing Corp., New York, NY, 1975.

[53] Bell, D. J., and Jacobson, D. H., Singular Optimal Control Problems, Academic Press, New York, NY, 1975.

[54] Schultz, R. L., and Zagalsky, N. R., "Aircraft Performance Optimization," Journal of Aircraft, Vol. 9, No. 2, February 1972, pp.108-114.

[55] Speyer, J. L., "On the Fuel Optimality of Cruise," Journal of Aircraft, Vol. 10, No. 12, December 1973, pp. 763-765.

[56] Ardema, M. D., "Singular Perturbations in Flight Mechanics," NASA TM X-62, 380, Aug. 1974; Revised July 1977.

[57] Ardema, M. D., "Characteristics of the Boundary-Layer Equations of the Minimum Time-to-Climb Problem," Proceedings of the Fourteenth Annual Allerton Conference on Circuit and System Theory, pp. 807-817, Sept. 29, 1976. 
[58] Ardema, M. D., "Linearization of the Boundary-Layer Equations of the Minimum Time-to-Climb Problem," Journal of Guidance and Control, Vol. 2, No. 5, pp.434436, Sept.-Oct. 1979.

[59] Price, D. B. and C. Gracy, "A Study of Altitude and Flight Path Angle Dynamics for a Singularly Perturbed Fuel Optimization Problem," Proceedings of the 1983 ACC, pp. 796-798.

[60] Gracy, C. and D. B. Price, "Altitude/Path-Angle Transitions in Fuel-Optimal Problems for Transport Aircraft," Proceedings of the 1983 ACC, pp. 519-525.

[61] Weston, A. R., Cliff, E. M., and H. J. Kelly, "Altitude Transitions in Energy Climbs," Automatica, Vol. 19, No. 2, pp. 199-202, 1983.

[62] Ardema, M. D, and L. Yang, "Interior Transition Layers in Flight-Path Optimization," Journal of Guidance and Control, Vol. 11, No. 1, pp. 13-18, Jan.Feb., 1988.

[63] Heiges, M. W., "A Helicopter Flight Path Controller Design via a Nonlinear Transformation Technique," Ph.D. Thesis to be published at The Georgia Institute of Technology, 1988.

[64] Brockett, R. W., "Nonlinear Systems and Differential Geometry," Proceedings of the IEEE, Vol. 64, pp 61-72, February 1976.

[65] Hunt, L. R., R. Su and G. Meyer, "Global Transformations of Nonlinear Systems," IEEE Transactions on Automatic Control, Vol. AC-28, No. 1, pp. 24-31, January 1983.

[66] Meyer, G., R. Su and L. R. Hunt, "Applications to Aeronautics fo the Theory of Transformations of Nonlinear Systems," NASA TM 84249, May 1982.

[67] Hunt, L. R., G. Meyer and R. Su, "Nonlinear Control of Aircraft," NASA TM $89225,1986$.

[68] Menon, P. K. A., A. J. Calise and S. K. M. Leung, "Guidance Laws for Spacecraft Pursuit-Evasion and Rendevous," Proceedings of the AIAA Guidance, Navigation, and Control Conference, pp. 688-697, 1988.

[69] Speyer, J. L., and A. E. Bryson, Jr., "Optimal Programming Problems with a Bounded State Space," AIAA Journal, Vol. 6, No. 8, pp. 1488-1491, August 1968.

[70] McIntyre, J. and B. Paiewonsky, "On Optimal Control with Bounded State Variables," in Advances in Control Systems: Theory and Applications, Vol. 5 , Academic Press, New York, NY, 1967.

[71] Anderson, J. D., Jr., Fundamentals of Aerodynamics, McGraw-Hill Co., New York, NY, 1982. 
[72] Anderson, J. D., Jr., Modern Compressible Flow with Historical Perspective, McGraw-Hill Co., New York, NY, 1982.

[73] Anderson, J. D., Jr., "A Survey of Modern Research in Hypersonic Aerodynamics," Proceedings of the ALAA $17^{\text {th }}$ Fluid Dynamics, Plasma Dynamics, and Lasers Conference, June 25-27, 1984, Snowmass, Colorado (AIAA-84-1578).

[74] McMahon, H. M., "Lecture Notes on Hypersonic Flow Theory," The Georgia Institute of Technology, School of Aerospace Engineering, 1987. 


\section{Appendix A}

\section{Prediction of Lift and Drag in Hypersonic Flow}

The flow features that dominate the aerodynamic behavior of atmospheric flight vehicles vary greatly with the flow velocity and the thermodynamic properties of the fluid medium. This variation is perhaps best quantified by means of the Mach number. The methods that may be employed in estimating aerodynamic forces in turn depend in large part on which flow features are dominant. For this reason it is convienient to divide the total flight regime into four regions, namely the subsonic, transonic, supersonic and hypersonic flow regimes. Table $\mathrm{A} 1$, on the following page, provides a definition of each regime and rules of thumb as to their relative boundaries [71].

There are, of course, other important ways of classifying flowfields. For example, flows in which the effects of viscosity, thermal conduction and mass diffusion are important are called viscous flows. Of note is the fact that surface pressure distributions, as well as aerodynamic lift and moments on some bodies can be accurately obtained by means of the assumption of inviscid flow. Another common assumption is that the fluid medium is a continium. This assumption is violated only for very low density flows, which occur at very high altitudes (above 200,000 feet) [72]. This appendix is dedicated to aerodynamic force predictions in the hypersonic flight regime. Methods suitable for developing subsonic and supersonic models of similar complexity are currently being investigated.

There is no clear dividing line between supersonic and hypersonic flow and the often quoted boundary of Mach 5 is, in reality, only a rule of thumb. Hypersonic flow is

formally defined as that regime where one or more of the following phenomena dominate the flow field [73]:

- Thin Shock Layers

- Entropy Layer

- Viscous Interaction

- High Temperature Flows

- Low Density Flows 
Table Al Definition of Flow Regions

Flow Region

Definition

Rule of Thumb

Subsonic-incompressible

Density is constant

$\mathrm{M}_{\infty}<0.3$

Subsonic-compressible

$\mathrm{M}<1$ everywhere

$\mathrm{M}_{\infty}<0.8$

Transonic

Mixed regions where $M<1$ and $M>1$

$0.8<\mathrm{M}_{\infty}<1.2$

Supersonic

$M>1$ everywhere

$\mathrm{M}_{\infty}>1.2$

Hypersonic

See text that follows

$\mathrm{M}_{\infty}>5.0$

As one would suspect, these effects - thin shock layers and hot chemically reacting gases - add great complexity to the analysis of supersonic flows. In fact, the solutions to such problems push the limits of current technology [73]. Modern hypersonic research is now dominated by the methods of computational fluid dynamics (CFD) and without such tools and the supercomputers on which they are exercised it is unlikely that the aerospace plane would ever be born [38].

Fortunately for our purposes (trajectory optimization) we find that viscous interaction, high temperature and low density effects may be disregarded. The approximate methods developed for inviscid hypersonic flow will allow us to adequately estimate lift and wave drag coefficients [73]. A major simplification results from the "Mach Number Independence Principle " which is illustrated in Fig. A1. This figure, reproduced from reference [73] and generated using the oblique shock relations, indicates that although the pressure ratio, $P_{1} / P_{2}$, is continuously decreasing, the pressure coefficient, $C_{p}$, is approximately constant above Mach 6 or 7 . Thus the force and moment coefficients 
obtained by integrating the pressure distribution over the body are also independent of Mach number in the hypersonic regime.

Additional simplifications accrue from the application of impact methods to represent the pressure distributions over the body and aerodynamic surfaces. Newtonian flow theory, which is further detailed at the end of this appendix, does not assume a continuim, but rather models the flow as a stream of discrete particles. It is then postulated that the normal component of momentum of each particle is destroyed upon impact with a body immersed in the flow whereas the tangential component is assumed to remain unchanged. The force exerted on a flat plate by the presence of the flow is then easily computed by using the conservation laws.

Since the wings, fins and control surfaces of our vehicle are thin, they can be approximated as flat plates of zero thickness. Simple Newtonian flow theory then provides a simple analytic means for predicting the pressure distribution on these surfaces as a fountain of angle of attack and independent of Mach numbers greater than about 5 . Of note, however, is the fact that at hypersonic speeds, the flow about a flat plate with a sharp leading edge "sees" a blunt nosed body due to the very rapid buildup of a thick boundary layer (i.e. viscous interaction). This is often referred to as the leading edge problem. Perhaps more to the point is the fact that all practical hypersonic vehicles have blunt noses and leading edges to reduce aerodynamic heating. Newtonian flow theory does not account for the additional drag due to a blunted leading edge when approximating a wing as a flat plate or the blunted nose of a fuselage. Blast wave theory, which is also further detailed at the end of this appendix, provides a fairly simple means for correcting the former method for these important leading edge and nose effects [74].

In some cases a combination of Newtonian flow and blast wave theories yields a highly accurate model of the pressure distribution on a body at hypersonic speeds. Figure A2, reproduced from reference [73], compares the pressure coefficients obtained using combined blast wave/Newtonian theory with flight data for the space shuttle. Remarkable agreement is achieved.

This technique has been applied to develop a simple three-dimensional aerodynamic representation of a slender hypersonic vehicle using a minimum number of geometric 
parameters. The resulting model, valid only in the hypersonic flight regime, can be adjusted in a straightforward fashion to simulate the geometry of a wide variety of aerospace plane configurations. This method has met with limited success when applied to the selected hypersonic research vehicle configuration. A three view drawing of the vehicle configuration was presented in Fig. 2 (of the main body of this report). The side, planform, and fin profiles were fit with straight line segments. Ten such segments yield an acceptable representation. The effects of wing incidence, fin cant, and propulsion modules

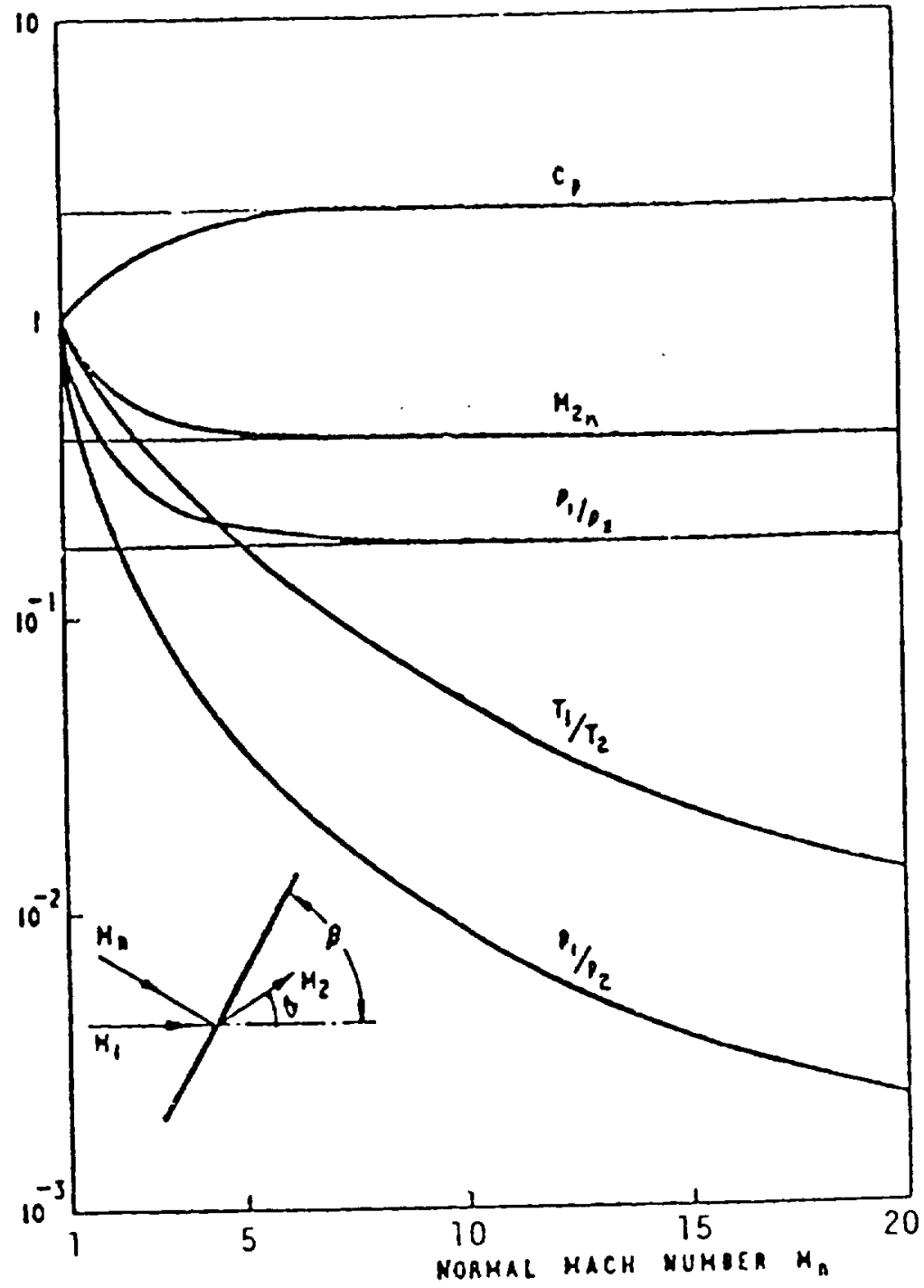

Figure A1 Mach Number Independence 


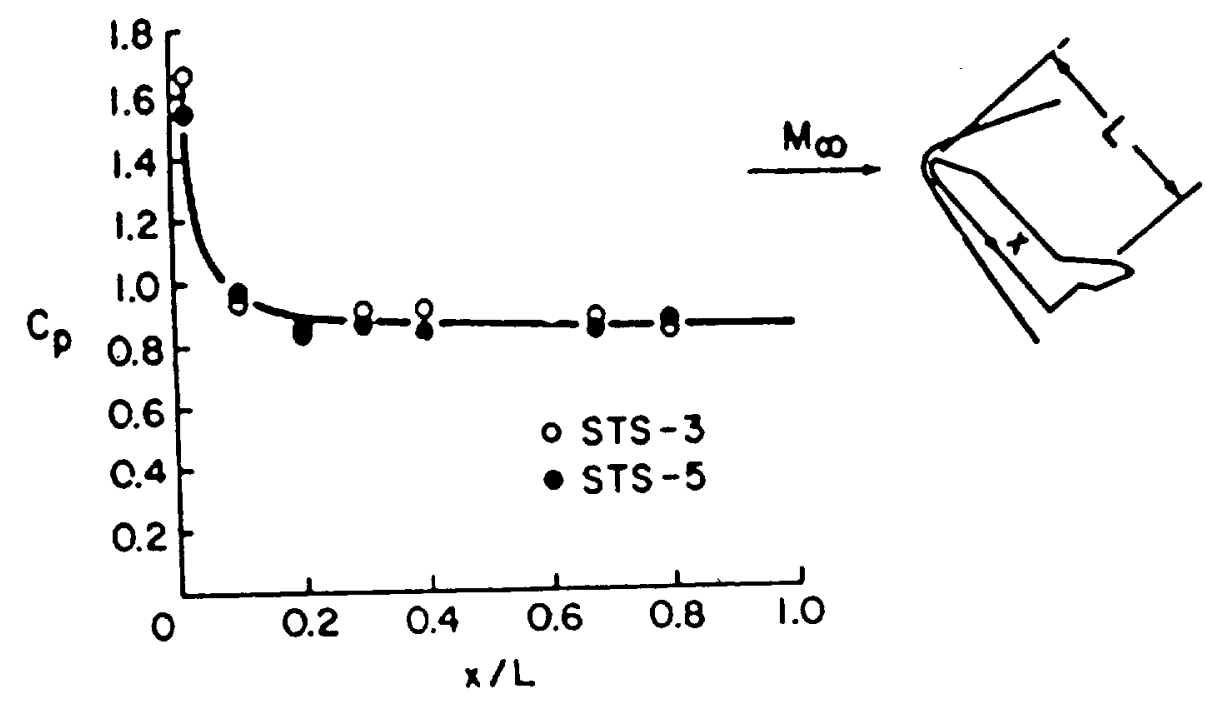

Figure A2 Comparison of pressure coefficients obtained with combined blast wave/Newtonian theory (solid line) with flight data for the space shuttle (circles). Windward centerline. Mach number of 21.6, angle of attack of $40^{\circ}$.

were incorporated. Blast wave theory was applied to the body to estimate the nose effects on the pressure distribution. Newtonian impact theory was used to correct this pressure distribution for yaw and pitch orientation angles (angle of attack and sideslip angle), and was also used to represent the wave lift and drag on the aerodynamic surfaces. Provision was made for differential deflection of the elevons. No attempt was made to correct for interference effects. Viscous drag was estimated by application of a simple skin friction coefficient. Figures $\mathrm{A} 3$ and $\mathrm{A} 4$ show the predicted drag polar and variation of $L / D$ ratio with $\mathrm{Cl}$ for the model described. Comparison with experimental results at Mach 6 [18], indicates reasonable agreement between theoretical and measured results. 
Figure A5 shows an important lateral stability effect and its dependence on the fin cant angle. The two curves representing 0 and 10 degree cant angles were generated by the method described above. Notice that the yaw moment coefficient versus sideslip angle exhibits a zero slope at zero sideslip. This results in poor lateral stability characteristics. This difficulty is alleviated by applying a cant angle to the fins as illustrated.

Figure A6 shows the predicted effects of elevon deflection on the longitudinal aerodynamic behavior as represented by the pitching moment coefficient, $\mathrm{Cm}$. Clearly the forces generated by elevon deflections are well represented, but the overall trend in $\mathrm{Cm}$ does not compare well with measured results. This is due to the very poor performance of the model in predicting vehicle lift. Figure A7 presents the trend of predicted and measured lift coefficient with increasing angle of attack for neutral elevon settings. Examination of this figure reveals the nature of the problem. The Newtonian result for the lift coefficient of a flat plate at angle of attack (see the end of this appendix for a derivation of this result) is:

$$
\mathrm{Cl}=2 \sin ^{2} \alpha|\cos \alpha|
$$

This trigometric behavior is clearly indicated in the predicted results by the inflection at zero angle of attack. On the other hand the measured results reflect a near linear relation between lift coefficient and angle of attack. Thus, although the model reasonably predicts drag as a function of lift, as required in the study of vehicle dynamics where lift is a control variable, the poor prediction of lift as a function of angle of attack precludes using the model to determine vehicle trim conditions. In order to allow work in trajectory optimization to continue, curve fits to the experimental data have been constructed and are to be used until a suitable model can be constructed. It is anticipated that the introduction of further geometric detail will result in a satisfactory model. The effect of body thickness, which has thus far been ignored in lift calculation, will provide additional lift generation at very low angles of attack. This mechanism is clearly evident in Fig. A5 by noting that the behavior of the vehicle with zero fin cant is similar to a body of parallel sides or zero thickness whereas the behavior of the vehicle with fin toe-in is similar to a tapered body. The result of the taper is more nearly linear behavior with changing incidence angle. 


\title{
Combined Newtonian Flow and Blast Wave Theory
}

\author{
Simple Newtonian [74]
}

This method provides simple but quite accurate estimates of surface pressure, exact as $M \infty$, the free stream Mach number, tends to infinity and $k$,the ratio of specific heats, tends to 1.0 .

Consider a body (in this case a flat plate) immersed in a fluid medium with free stream Mach number much greater than 1. We consider the fluid not as a continium, but as composed of discrete particles that do not interact with one another. This was Newton's flow model. Newton postulated that the normal component of momentum of each fluid particle was destroyed upon impact with a body immersed in the flow. The tangential component of velocity was assumed to remain unchanged. Figure A.8 illustrates this model. The force exerted on the flat plate by the presence of the flow can be computed by using the conservation laws. Newton used this flow model in an attempt to explain the drag of projectiles. At low speeds this model is very poor (it predicts that $\mathrm{Cl}$ is proportional to $\alpha^{2}$ when really $\mathrm{Cl}$ is proportional to $\alpha$ ), but at very high speeds (i.e. in the hypersonic flow regime) it is quite accurate.

Using Newton's flow model, it can be shown that the pressure coefficient on a flat surface with $M_{\infty} \gg 1$ is approximated by:

$$
\mathrm{Cp}=2 \sin 2 \alpha
$$

This relation is exact as $M_{\infty}$ tends to infinity and $k$ tends to 1.0 which in reality is never the case. This approximation is good for high Mach numbers however, continually improving with Mach number till $M>10$ or so. Note that for air $k=1.4$, but for $M_{\infty} \gg 1, k$ tends to decrease towards 1.0 as desired due to increasing temperature and its affects such as ionization. It is assumed that the pressure exerted on the surface of the body is zero everywhere in the aerodynamic shadow (see Fig. A.8). 


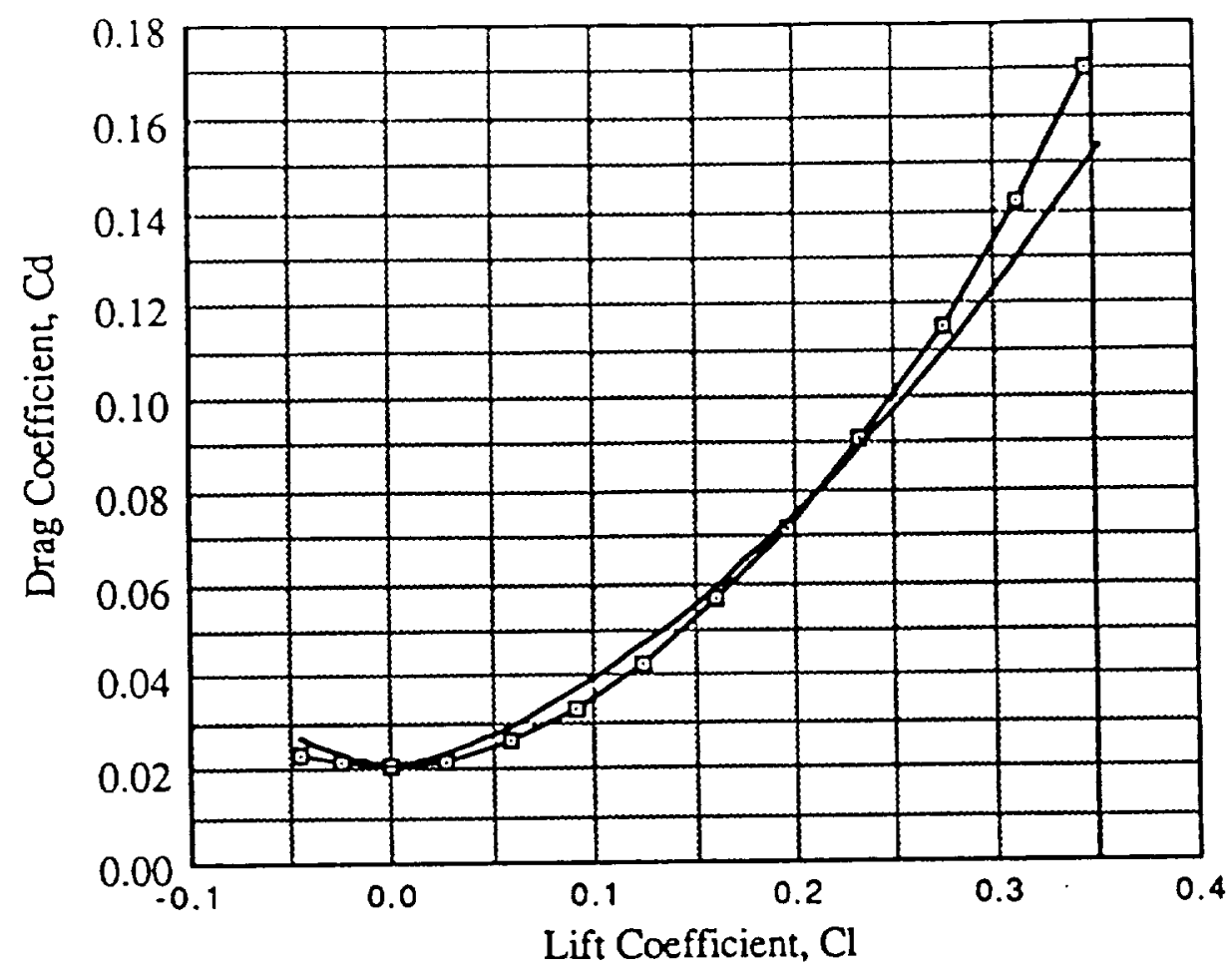

- Cdexp.

- Cd

Figure A3 Drag Polar, predicted and measured values at $M=6.0$ (neutral olevenn)

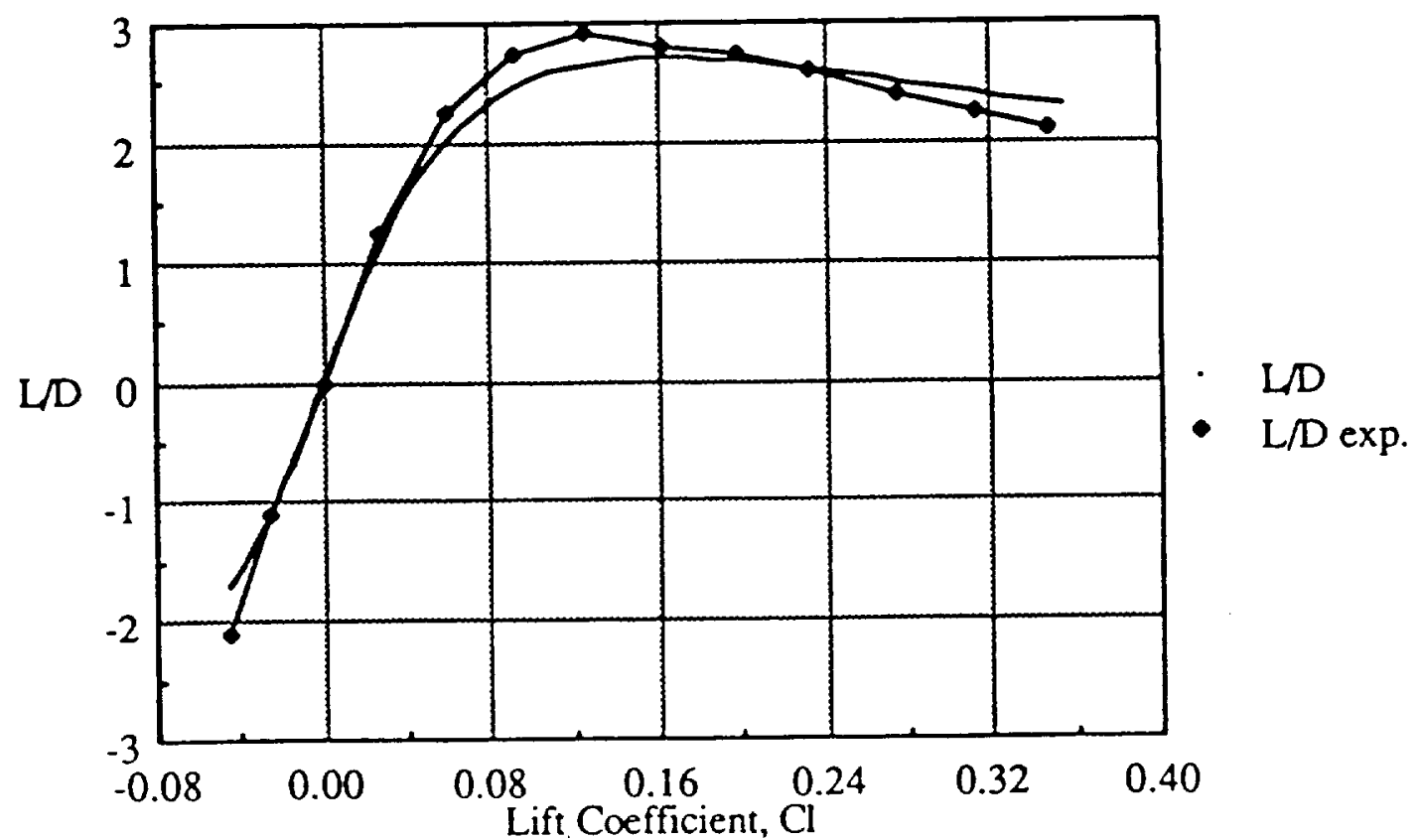

Figure A4 Lift to Drag Ratio, predicted and measured values at $M=6.0$ (neutral elevon) 


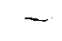

$-$

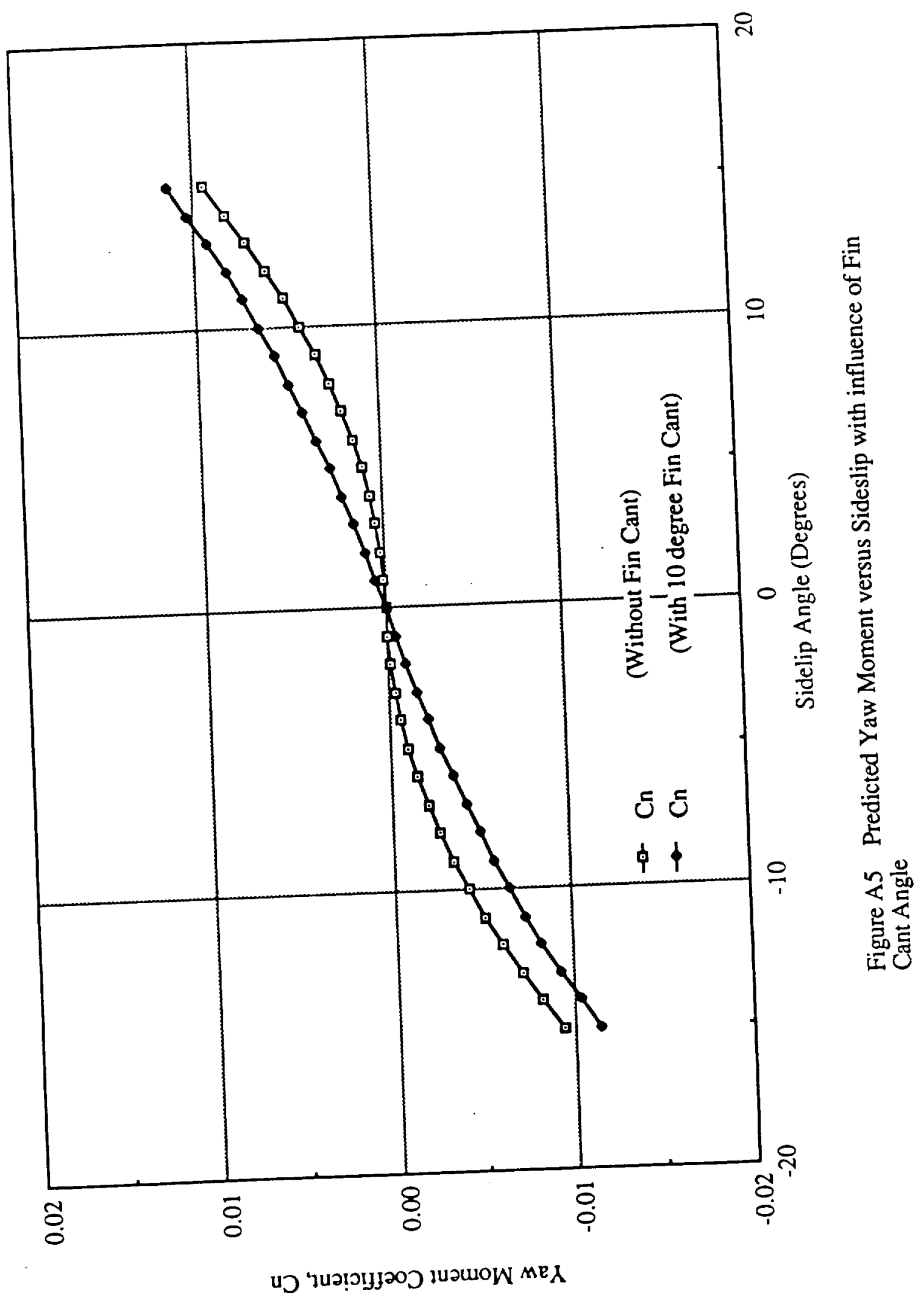


$\bar{u} \stackrel{\frac{\dot{0}}{x}}{u}$

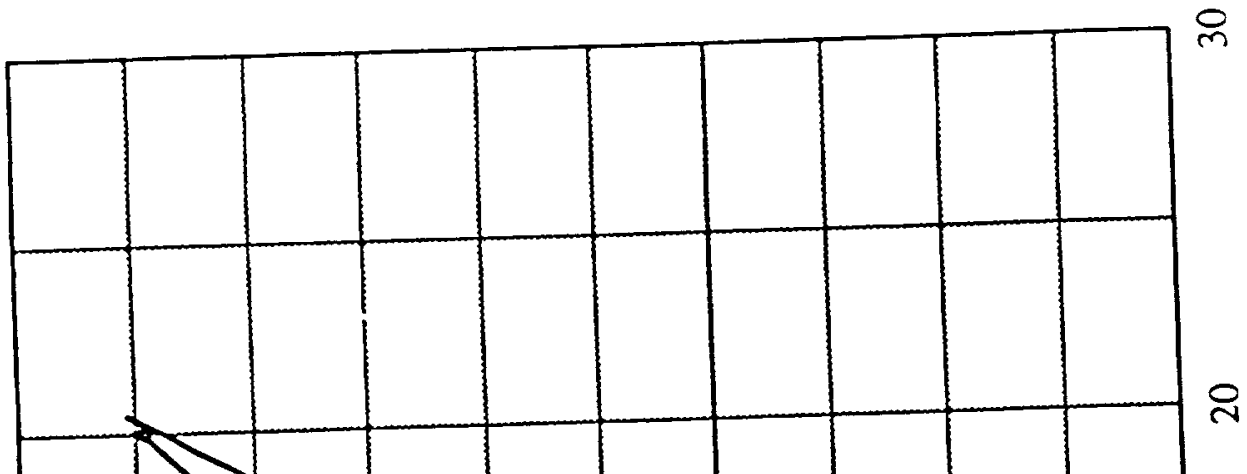

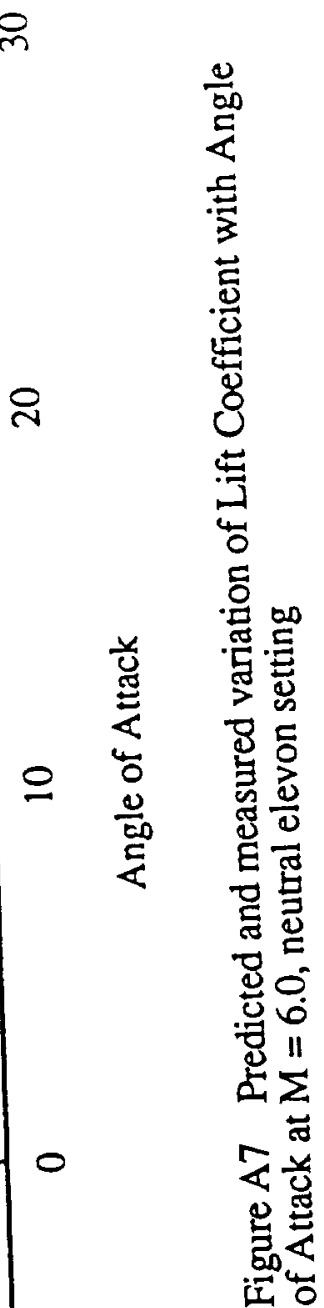

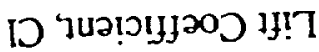


Let us apply simple Newtonian theory to estimate the lift and drag of a flat plate at angle of attack in hypersonic flight. Referring to Fig. A.9, the force per unit span on the plate is given by:

$$
F^{\prime}=(c) P_{\text {lower }}-(c) P_{\text {upper }}
$$

i.e. the net force on the plate (the prime denotes per unit span) is equal to the pressure difference between the upper and lower surfaces times multiplied by the surface area.

Now, adding and subtracting $P_{\infty}$ (pressure in the free stream) we can force this relation into a form in terms of the pressure coefficient, defined as:

$$
C_{p}=\left(P-P_{\infty}\right) /\left(1 / 2 \rho_{\infty} V_{\infty}^{2}\right)
$$

We then have:

$$
F^{\prime}=\left[\left(P_{1}-P_{\infty}\right)-\left(P_{u}-P_{\infty}\right)\right] c
$$

where $P_{u}-P_{\infty}$ is approximately zero since the upper surface is in the aerodynamic shadow.

Then

$$
C_{F^{\prime}}=F /\left(1 / 2 \rho_{\infty} V_{\infty}^{2} c\right)=\left(P_{1}-P_{\infty}\right) /\left(1 / 2 \rho_{\infty} V_{\infty}^{2}\right)=C_{P l}=2 \sin 2 \alpha
$$

where $\mathrm{CF}_{\mathrm{F}}$ denotes a non-dimensional force coefficient. Resolving this force coefficient into lift and drag components we have:

$$
\begin{aligned}
& \mathrm{Cl}^{\prime}=2 \sin 2 \alpha|\cos \alpha| \\
& \mathrm{Cd}^{\prime}=2 \sin 2 \alpha|\sin \alpha|
\end{aligned}
$$




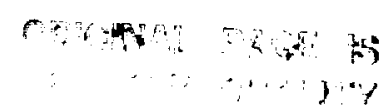

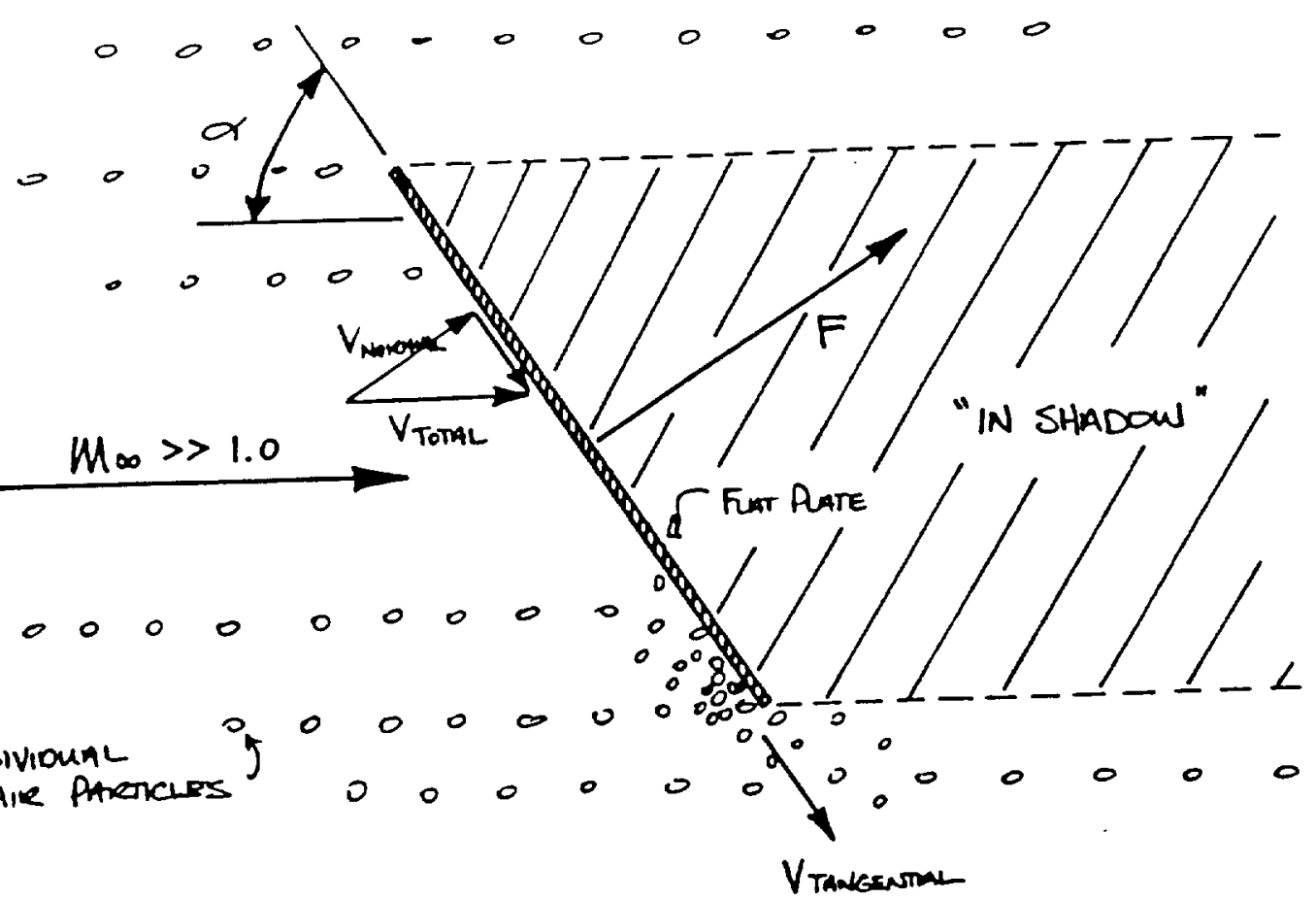

Figure A8 Newton's Flow Model

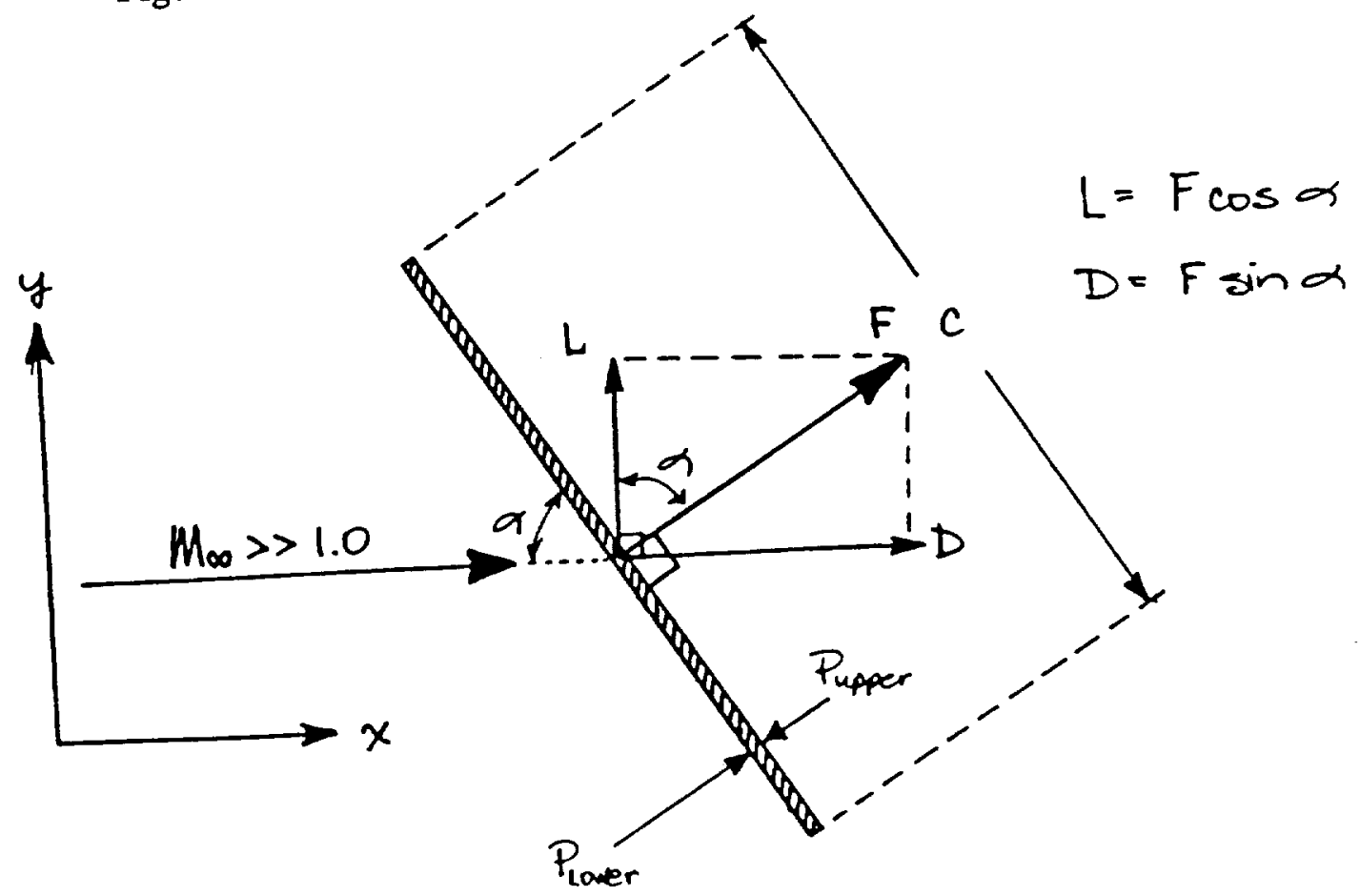

Figure A9 Components of Lift and Drag for a Flat Plate 


\section{Blast Wave Theory [74]}

We may consider blast wave theory as a variation of the hypersonic equivalence principle which states: "A steady 3-D hypersonic flow over a slender body is equivalent to an unsteady flow in one less dimension." For a severely blunted body we may approximate the flow situation by assuming all of the drag is concentrated at the nose(see Fig. A.10). We may then reduce a 3-D blunt-nosed body problem to that of a 2-D circular shock which grows with time (see Fig. A.11).

Much work was done on the theory of circular and spherical blast waves in the 1940's 1950 's. These results can be used to approximate the shock shape for blunt-nosed bodies in hypersonic flow fields by equating explosive energy with the drag of the body. Note that this technique requires knowledge of $\mathrm{C}_{\mathrm{d}}$ for the body. Once the shock shape is determined, the oblique shock relations are used to estimate the surface pressure distributions.

The blast wave solutions are based upon the assumption that flow similarity exists, i.e. it is assumed that

$$
P(r) / P(R)=\text { function }(r / R)
$$

where $r$ is the distance to a point of interest in the field and $R$ is the distance from the source to the shock. Very close to the origin of an expansion we expect extremes of temperature and pressure, thus our assumption of similarity will not hold very close to the nose. For this reason we do not expect good results from blast wave theory right at the nose. Our solution is also based on the assumption that the shock is quite strong which is not true as we move far back from the nose. Thus we do not expect the blast wave results to be good far back from the nose.

We will now develop the blast wave solution for a blunt nosed cylinder (i.e. an axisymmetric body) After noting that this solution is only available for zero angle of attack, a correction for angle of attack based on simple Newtonian will be appended. Note that at hypersonic speeds, the flow about a flat plate with a sharp leading edge "sees" a blunt nosed body due to the very rapid buildup of a thick boundary layer (i.e. viscous interaction). This is of ten referred to as the leading edge problem. We may find it necessary 


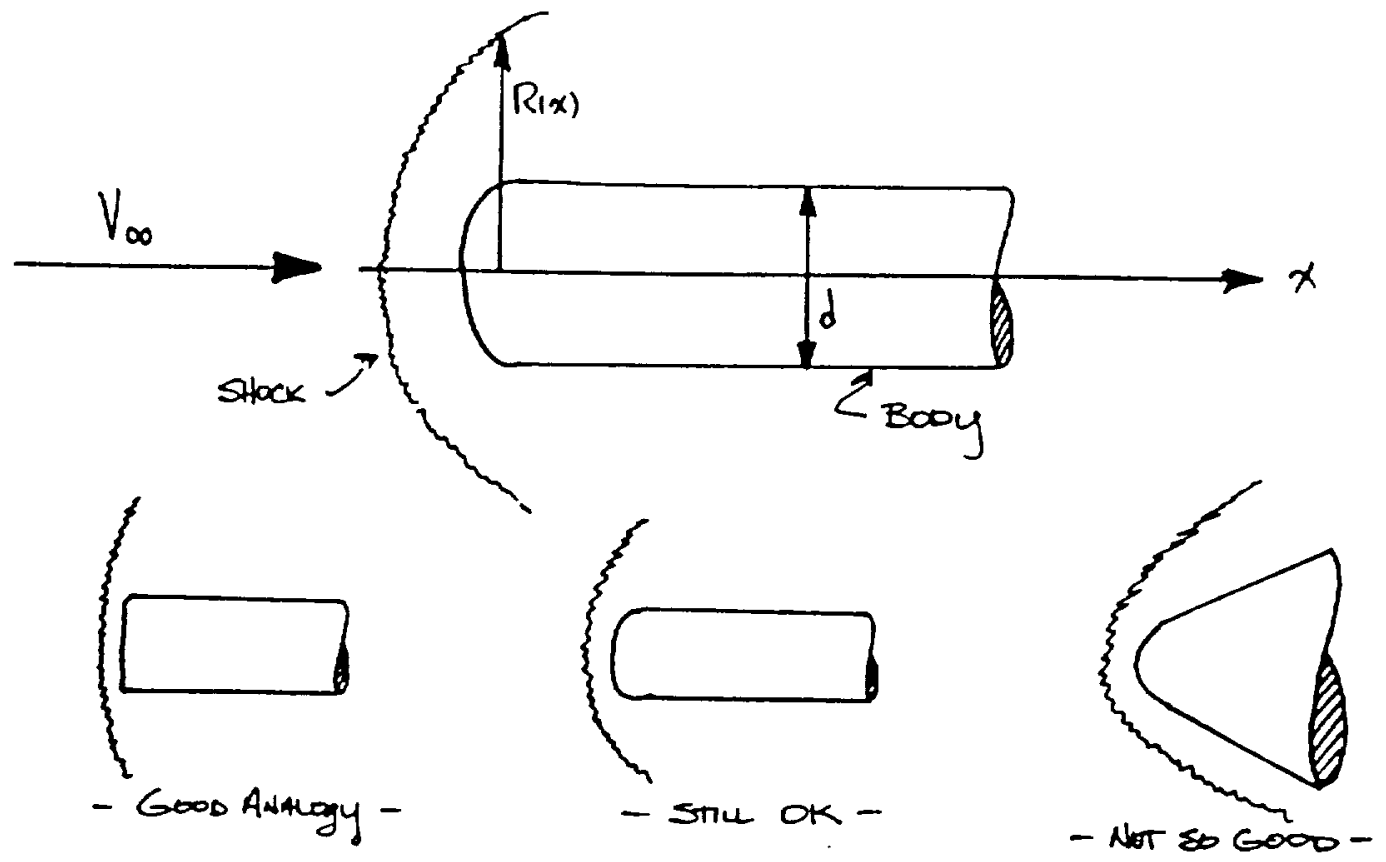

Figure A10 Severity of Bluntness

2-D Ropresentanion, RAOMs of Clecuute SHock Gaws wint Time

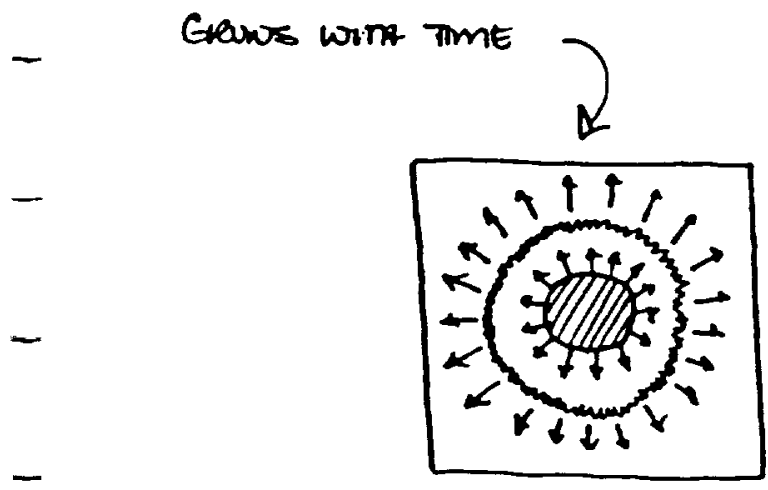

Booy passes thru PuANE As TIME EVOUVES

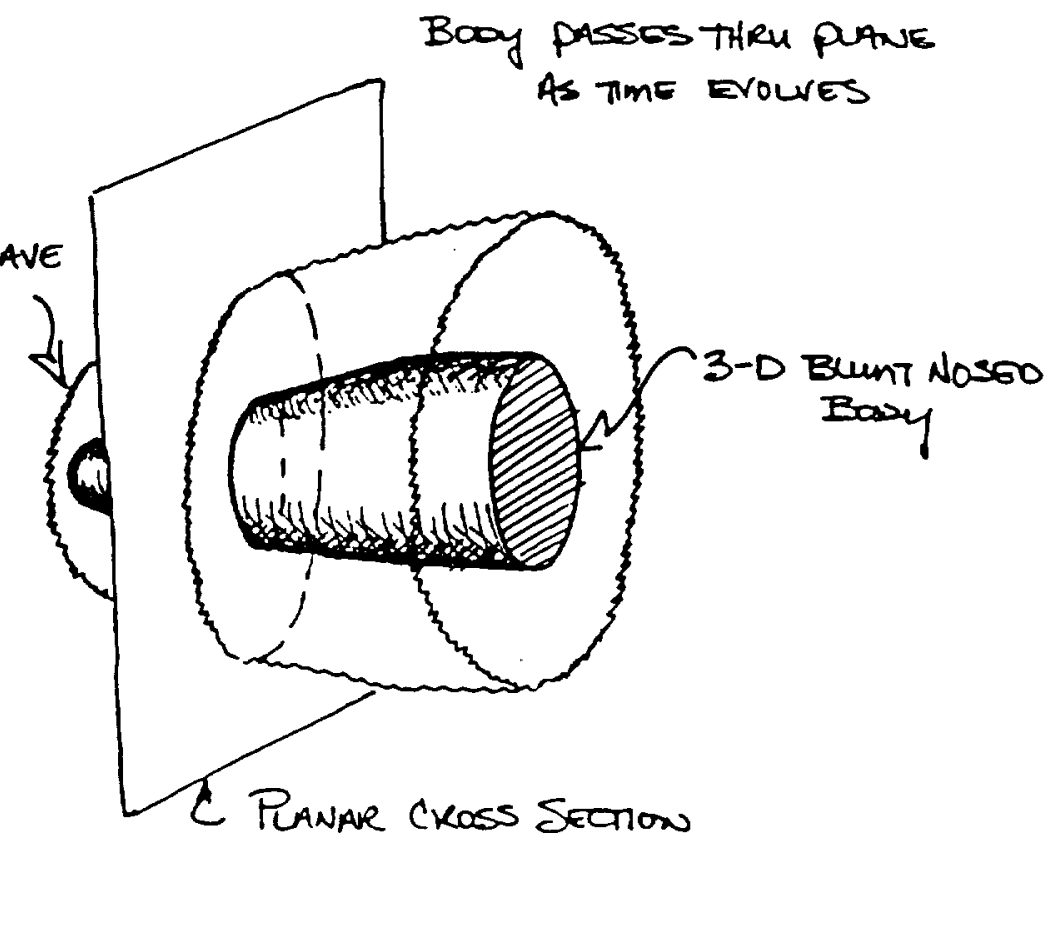

Figure A11 2-D Representation of 3-D Flow Problem 
to employ blast wave theory to estimate the additional drag due to a blunted leading edge when approximating a wing as a flat plate.

\section{Blunt-Nosed Cylinder at Zero Angle of Attack}

For an expanding shock from a finite release of energy in a gas at rest blast wave theory gives the radius of the shock as:

$$
\begin{aligned}
& R(t)=f(k, n)\left[E / \rho_{\infty}\right]^{(1 /[3+n]) t(2 /[3+n])}
\end{aligned}
$$

where $f(k, n)=$ constant from numerical integration which

$k=$ ratio of specific heats

$\mathrm{n}=$ characteristic number

$$
\begin{aligned}
& \mathrm{n}=0 ; \text { planar case } \\
& \mathrm{n}=1 ; \text { cylindrical case } \\
& \mathrm{n}=2 ; \text { spherical case }
\end{aligned}
$$

Now we make a transformation from time to space:

$$
\begin{aligned}
& t \text { becomes } x / V_{\infty} \text { and } R(t) \text { becomes } R(x) \\
& R(x)=f(k, n)\left[E / \rho_{\infty}\right]^{(1 / \pi 3+n]) \times(2 /[3+n])}
\end{aligned}
$$

This gives the shock shape as a function of the longitudinal body coordinate $x$ (see Fig.

A.3).

Next we equate energy in the blast problem to drag in the aerodynamic problem.

$$
E=D=q_{\infty} C_{d}\left[\pi d^{2} / 4\right] \quad(n=1)
$$

Here $d$ is the diameter of the cylinder and $q_{\infty}$ is dynamic pressure. Then

$$
R / d=f(k, 1)\left\{\left[1 / 2 \rho_{\infty} V_{\infty} 2 C_{d}\left(\pi d^{2} / 4\right)\right] /\left[\rho_{\infty} d^{4}\right]\right\}^{1 / 4}\left[x / V_{\infty}\right]^{1 / 2}
$$

or

$$
R / d=f_{o}(k) C_{d}^{1 / 4}[x / d]^{1 / 2} \quad \text { where } f_{o}(k) \text { is given as } 0.795
$$


Thus if we know the value of $C_{d}$, we can get an estimate of the shock shape. We can then use the oblique shock relations to get $C_{p}$ just behind the shock. Finally we assume that $\mathrm{C}_{\mathrm{p} \text { shock }}$ is approximately equal to $\mathrm{C}_{\mathrm{p}}$ body.

$$
P_{S} / P_{\infty}=[2 k /(k+1)] M_{\infty}^{2} \sin ^{2} \beta-[(k-1) /(k+1)]
$$

We can neglect the second term of (13) for strong shocks and since for $M_{\infty} \gg 1$ the shock lies back very near the body we may assume:

$$
\sin \beta=\tan \beta=\beta=d R / d x=d(R / d) / d(x / d)
$$

Thus

$$
\sin ^{2} \beta=\{d / d(x / d)[\text { Eqn. (A.12) }\} 2
$$

So that

$$
P_{S} / P_{\infty}=g(k) M_{\infty}^{2}\left[C_{d}^{1 / 2 /(x / d)}\right]=P_{b o d y} / P_{\infty}
$$

where $g(k)$ is a constant given as 0.067 . Now by definition:

$$
C_{p}=2 /\left(\mathrm{kM}_{\infty}^{2}\right)\left[\mathrm{P} / \mathrm{P}_{\infty}-1\right]
$$

If we neglect the 1 as small compared to the pressure ratio then we may write:

$$
C_{p}=2 g(k) / k\left[C_{d}^{1 / 2 /(x / d)][L / L]}\right.
$$

where $L$ is total body length. Taking $k$ to be 1.4 the quantity $2 \mathrm{~g}(\mathrm{k}) / \mathrm{k}$ becomes 0.096 and we have the result:

$$
C_{p}=0.096 C_{d}^{1 / 2}(L / x)(L / d)-1
$$

Note that this results assumes an angle of attack of zero. We may correct for incidence angle using the simple Newtonian result derived for a flat plate:

$$
C_{p}=0.096 C_{d}^{1 / 2}(L / x)(L / d)-1+2 \sin 2 \alpha
$$




\section{Appendix B}

\section{Minimization of a Hamiltonian Function with One Unknown Costate and One Costate of Known Sign}

This appendix generalizes Appendix $\mathrm{E}$ of reference [40] and as such documents a method for minimizing a Hamiltonian with one unknown costate and one costate of known sign.

Consider the Hamiltonian function

$$
H^{*}(x)=\mu f(x)+\lambda g(x)=0
$$

where the sign of $\mu$ is known. It is of interest to minimize this function with respect to $x$. Alternatively we may consider an equivalent operation exercised on the function $H(x)$ with respect to $x$ where $H(x)$ is defined as,

$$
H(x) \equiv H^{*}(x) / \mu=f(x)+\eta g(x)=0
$$

and for convenience we have defined,

$$
\eta=\lambda / \mu
$$

Now, equivalent to minimizing $H^{*}(x)$ with respect to $x$ we have the following operations

$$
\begin{array}{ll}
\min _{x} H & \text { if } \mu>0 \\
\max _{x} H & \text { if } \mu<0
\end{array}
$$


Sufficient conditions for the existence of a minimum are (where the subscript notation denotes partial differentiation)

$$
\begin{aligned}
& H_{x}=f_{x}+\eta g_{x}=0 \\
& H_{x x}=f_{x x}+\eta g_{x x}\left\{\begin{array}{l}
>0 \text { if } \mu>0 \\
<0 \text { if } \mu<0
\end{array}\right.
\end{aligned}
$$

In a free final time problem where $\mathrm{H}^{*}$ does not explicitly depend on time, it is also necessary that $\mathrm{H}^{*}$, and thus $H$, be zero. Using (B.2) to solve for $\eta$ leads to

$$
\eta=-f / g \quad g \neq 0
$$

Using (B.7) in (B.5) and (B.6) we obtain the conditions:

$$
\begin{aligned}
& H_{x}=f_{x}+(-f / g) g_{x}=0 \\
& H_{x x}=f_{x x}+(-f / g) g_{x x}\left\{\begin{array}{l}
>0 \text { if } \mu>0 \\
<0 \text { if } \mu<0
\end{array}\right.
\end{aligned}
$$

Now define the function

$$
L=g / f
$$

Next take the first and second partial derivatives of $\mathrm{L}$ with respect to $\mathrm{x}$

$$
\begin{aligned}
& L_{x}=\left(f g_{x}-g f_{x}\right) / f^{2} \\
& L_{x x}=\left(f g_{x x}-g f_{x x}\right) / f^{2}-2 f_{x} L_{x} / f
\end{aligned}
$$

Setting (B.11) equal to zero yields the same conditions as given by (B.5) and (B.7). 
Condition (B.9) can be rewritten as

$$
\left.\left.\begin{array}{l}
\left.g f_{x x}-f g_{x x}>0 \quad \text { if } g>0\right) \\
g f_{x x}-f g_{x x}<0 \quad \text { if } g<0
\end{array}\right\} \begin{array}{lll}
g f_{x x}-f g_{x x}<0 & \text { if } g>0 \\
\text { Using } L_{x}=0 \text { in (B.12) we have } & g f_{x x}-f g_{x x}>0 & \text { if } g<0
\end{array}\right\}^{\mu<0}
$$

$$
L_{x x}=\left(g f_{x x}-f g_{x x}\right)\left(-1 / f^{2}\right)
$$
Since the last term in (B.14) is always negative, the following conditions are equivalent to
(B.13):

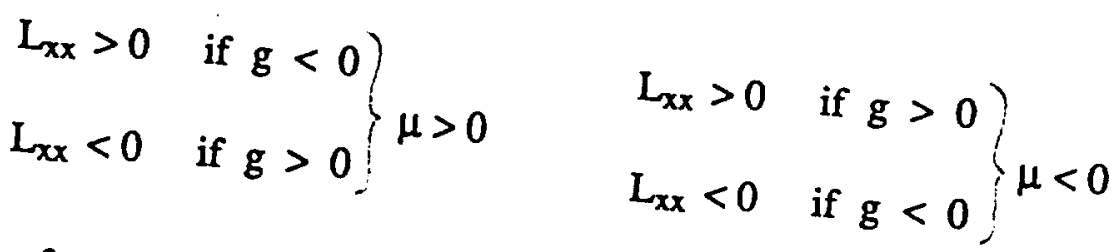

From the foregoing, we can conclude that the conditions

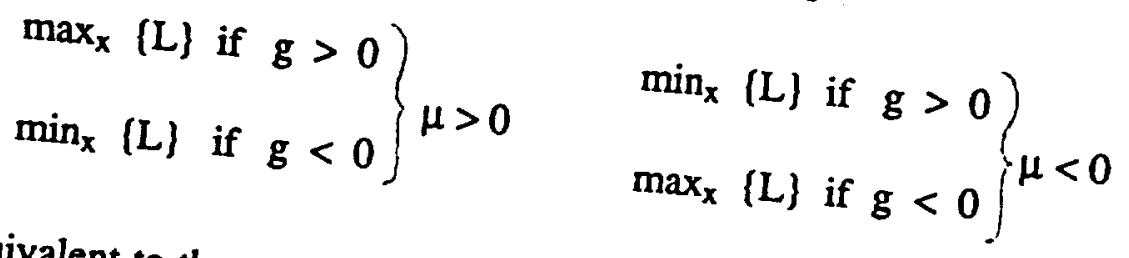
concurrently. 